\title{
METRICS WITH FOUR CONIC SINGULARITIES AND SPHERICAL QUADRILATERALS
}

\author{
ALEXANDRE EREMENKO, ANDREI GABRIELOV, AND VITALY TARASOV
}

\begin{abstract}
A spherical quadrilateral is a bordered surface homeomorphic to a closed disk, with four distinguished boundary points called corners, equipped with a Riemannian metric of constant curvature 1, except at the corners, and such that the boundary arcs between the corners are geodesic. We discuss the problem of classification of these quadrilaterals and perform the classification up to isometry in the case that two angles at the corners are multiples of $\pi$. The problem is equivalent to classification of Heun's equations with real parameters and unitary monodromy.
\end{abstract}

\section{INTRODUCTION}

Let $S$ be a compact Riemann surface, and $a_{0}, \ldots, a_{n-1}$ a finite set of points on $S$. Let us consider a conformal Riemannian metric on $S$ of constant curvature $K \in\{0,1,-1\}$ with conic singularities at the points $a_{j}$. This means that in a local conformal coordinate $z$ the length element of the metric is given by the formula $d s=\rho(z)|d z|$, where $\rho$ is a solution of the differential equation

$$
\Delta \log \rho+K \rho^{2}=0 \quad \text { in } S \backslash\left\{a_{0}, \ldots, a_{n-1}\right\},
$$

and $\rho(z) \sim|z|^{\alpha_{j}-1}$, for the local coordinate $z$ which is equal to 0 at $a_{j}$. Here $\alpha_{j}>0$, and $2 \pi \alpha_{j}$ is the total angle around the singularity $a_{j}$.

The general problem is how many such metrics exist with prescribed $a_{j}$ and $\alpha_{j}$.

This question goes back to the nineteenth century, and for the history we refer to [16, 41]. A complete answer to this question is known when $K \leq 0,22,34,37,48$, but very little is known on the case $K>0$.

One necessary condition that one has to impose on these data follows from the Gauss-Bonnet theorem: the quantity

$$
\chi(S)+\sum_{j=0}^{n-1}\left(\alpha_{j}-1\right) \quad \text { has the same sign as } K .
$$

Here $\chi$ is the Euler characteristic. Indeed, this quantity multiplied by $2 \pi$ is equal to the integral curvature of the smooth part of the surface.

The result of Troyanov [48] that applies to the case $K=1$ is the following:

Received by the editors July 7, 2015 and, in revised form, March 11, 2016 and March 12, 2016. 2010 Mathematics Subject Classification. Primary 30C20, 34M03.

Key words and phrases. Surfaces of positive curvature, conic singularities, Heun equation, Schwarz equation, accessory parameters, conformal mapping, circular polygons.

The first author was supported by NSF grant DMS-1361836.

The second author was supported by NSF grant DMS-1161629. 
Let $S$ be a compact Riemann surface, $a_{0}, \ldots, a_{n-1}$ points on $S$, and let $\alpha_{0}, \ldots$, $\alpha_{n-1}$ be positive numbers satisfying

$$
0<\chi(S)+\sum_{j=0}^{n-1}\left(\alpha_{j}-1\right)<2 \min \left\{1, \min _{0 \leq j \leq n-1} \alpha_{j}\right\} .
$$

Then there exists a conformal metric of positive curvature 1 on $S$ with conic singularities at $a_{j}$ and angles $2 \pi \alpha_{j}$.

F. Luo and G. Tian [30] proved that if the condition $0<\alpha_{j}<1$ is satisfied, then (1.3) is necessary and sufficient, and the metric with given $a_{j}$ and $\alpha_{j}$ is unique.

In general, the right hand side inequality in (1.3) is not a necessary condition, and the metric may not be unique.

In this paper, we only consider the simplest case when $S$ is the sphere, so $\chi(S)=$ 2. For metrics on tori we refer to the recent works [6, 7].

The problem of description and classification of conformal metrics of curvature 1 with conic singularities on the sphere has applications to the study of certain surfaces of constant mean curvature [9, 10, 19], and to several other questions of geometry and physics 39,47 .

The so-called "symmetric case" is interesting and important. Suppose that all singularities belong to a circle on the sphere $S$, and we only consider the metrics which are symmetric with respect to this circle. Then the circle splits $S$ into two symmetric disks. Each of them is a spherical polygon, (surface) for which we state a formal definition:

Definition 1.1. A spherical $n$-gon is a closed disk with $n$ distinguished boundary points $a_{j}$ called the corners, equipped with a conformal Riemannian metric of constant curvature 1 everywhere except the corners, and such that the sides (boundary arcs between the corners) are geodesic. The metric has conic singularities at the corners.

In 11, 50, all possibilities for spherical triangles are completely described; see also [19] where a minor error in [11, Theorem 2] was corrected. In the case of triangles, the metric is uniquely determined by the angles when none of the $\alpha_{j}$ is an integer.

The case when all $\alpha_{j}$ are integers, and $n$ is arbitrary, is also well understood. In this case, the line element of the metric has the global representation

$$
d s=\frac{2\left|f^{\prime}\right||d z|}{1+|f|^{2}}
$$

where $f$ is a rational function. The singular points $a_{j}$ are the critical points of $f$, $\alpha_{j}-1$ is the multiplicity of the critical point $a_{j}$, and $\alpha_{j}$ is the local degree of $f$ at $a_{j}$.

Thus the problem with all integer $\alpha_{j}$ is equivalent to describing rational functions with prescribed critical points [12, 15, 18, 21, 32, 42].

Almost nothing is known in the case when some of the $\alpha_{j}$ are not integers, the number of singularities is greater than 3 , and the right-hand side inequality in (1.3) is violated 1

\footnotetext{
${ }^{1}$ After the first version of this work was posted on the arXiv, preprint 31 appeared, where the authors find the general necessary and sufficient conditions on the angles $\alpha_{j}$ of a metric on the sphere with some singularities $a_{j}$.
} 
In this paper we begin an investigation of the case $n=4$, with the emphasis on the symmetric case.

In the symmetric case, we may assume without loss of generality that the circle is the real line $\mathbf{R} \cup\{\infty\}$. The real line splits the sphere $S$ into two symmetric spherical $n$-gons with the real corners $a_{j}$ and the angles $\pi \alpha_{j}$ at the corners.

Thus we arrive at the problem of classification of spherical quadrilaterals (surfaces). We study this problem using two different methods. One of them is the geometric method of F. Klein 28, who classified spherical triangles. Klein classified not only spherical triangles with geodesic sides, but also circular triangles, whose sides have constant geodesic curvature (that is, locally they are arcs of circles). A modern paper which uses Klein's approach to triangles is [52].

Classification of triangles permitted Klein to obtain exact relations for the numbers of zeros of hypergeometric functions on the intervals between the singular points on the real line. Van Vleck [51] extended this approach of Klein, using the same geometric method, and obtained exact inequalities for the numbers of zeros of hypergeometric functions in the upper and lower half-planes. Hurwitz 25] re-proved these results with a different, analytic method.

We hope that our results can be used to obtain information about solutions of Heun's equation, in the same way as Klein obtained information about solutions of the hypergeometric equation.

Klein's classification of triangles was partially extended to arbitrary circular quadrilaterals (not necessarily geodesic ones) in the work of Schönflies 43, 44, and Ihlenburg [26, 27]. They considered a certain geometric reduction process of cutting a circular quadrilateral into a simpler one. Then they classified the irreducible quadrilaterals up to conformal automorphisms of the sphere. Thus they obtained an algorithm which permits one to construct all circular quadrilaterals. Using this algorithm, Ihlenburg derived relations between the angles and sides of a circular quadrilateral. However this algorithm falls short of a complete classification. In particular, a quadrilateral with prescribed angles and sides is not unique. Moreover, it seems difficult to single out geodesic quadrilaterals in the construction of Schönflies and Ihlenburg.

We use a somewhat different approach which consists of associating to every spherical geodesic quadrilateral a combinatorial object which we call a net, thus reducing the classification to combinatorics. Then we solve this combinatorial problem and obtain a classification of spherical quadrilaterals up to isometry. Our approach can also be applied to general (non-geodesic) circular quadrilaterals.

The boundary of a spherical polygon is a closed curve on the sphere, consisting of geodesic pieces. The angles of such a curve at the corners make sense only modulo $2 \pi$. All possible sequences of angles have been described by Biswas [3]; see also [5]. These inequalities on the angles give necessary but not sufficient conditions that the angles of a spherical polygon (surface) must satisfy.

Our second method is a direct study of Heun's equation. Classification of spherical quadrilaterals can be stated in terms of a special eigenvalue problem for this equation. This method leads to complete results when the eigenvalue problem can be solved algebraically.

The contents of the paper are the following. In section2, we recall the connection of the problem with Heun's equation, and recall the results on Heun's equation related to our problem. 
In section 3 we begin the study of the case when two of the $\alpha_{j}$ are integers and two others are not. (If three of the $\alpha_{j}$ are integers, then all four must be integers.) Complete classification for this case is obtained in the remaining sections. Sections 3[5] are based on a direct study of the eigenvalue problem for Heun's equation. The results are illustrated with numerical examples in section 16. Sections 6. 14] are based on geometric and combinatorial methods. The case when three angles are non-integers is treated in [17.

Acknowledgment. We thank the referee whose remarks improved the exposition of this paper.

\section{Connection With Linear differential equations}

Let $(S, d s)$ be the Riemann sphere equipped with a metric with conic singularities. Every smooth point of $S$ has a neighborhood which is isometric to a region on the standard unit sphere $\mathbf{S}$; let $f$ be such an isometry. Then $f$ has an analytic continuation along every path in $S \backslash\left\{a_{0}, \ldots, a_{n-1}\right\}$, and we obtain a multi-valued function which is called the developing map. The monodromy of $f$ consists of orientation-preserving isometries (rotations) of $\mathbf{S}$, so the Schwarzian derivative

$$
F(z):=\frac{f^{\prime \prime \prime}}{f^{\prime}}-\frac{3}{2}\left(\frac{f^{\prime \prime}}{f^{\prime}}\right)^{2}
$$

is a single valued function.

The developing map is completely characterized by the properties that it has an analytic continuation along any curve in $S \backslash\left\{a_{1}, \ldots, a_{n}\right\}$, has asymptotics $\sim c\left(z-a_{j}\right)^{\alpha_{j}}, z \rightarrow a_{j}, c \neq 0$, and has $P S U(2)=S O(3)$ monodromy. It is possible that two such maps with the same $a_{j}$ and $\alpha_{j}$ are related by post-composition with a fractional-linear transformation. The metrics arising from such maps will be called equivalent. Following [19, we say that the metric is reducible if its monodromy group is commutative (which is equivalent to all monodromy transformations having a common fixed point). In the case of irreducible metrics, each equivalence class contains only one metric. For reducible metrics, the equivalence class is a one-parameter family when the monodromy is non-trivial and a three-parametric family when monodromy is trivial.

The asymptotic behavior of $f$ at the singular points $a_{j}$ implies that the only singularities of $F$ on the sphere are double poles, so $F$ is a rational function, and we obtain the Schwarz differential equation (2.1) for $f$.

It is well known that the general solution of the Schwarz differential equation is a ratio of two linearly independent solutions of the linear differential equation

$$
y^{\prime \prime}+P y^{\prime}+Q y=0, \quad f=y_{1} / y_{0},
$$

where

$$
F=-P^{\prime}-P^{2} / 2+2 Q \text {. }
$$

For example one can take $P=0$; then $Q=F / 2$. Another convenient choice is to make all poles but one of $P$ and $Q$ simple. When $n=3$, equation (2.2) is equivalent to the hypergeometric equation, and when $n=4$ to Heun's equation [40].

The singular points $a_{j}$ of the metric are the singular points of the equation (2.2). These singular points are regular, and to each point correspond two exponents $\alpha_{j}^{\prime}>\alpha_{j}^{\prime \prime}$, so that $\alpha_{j}=\alpha_{j}^{\prime}-\alpha_{j}^{\prime \prime}$. If $\alpha_{j}$ is an integer for some $j$, we have an additional condition of the absence of logarithms in the formal solution of (2.2) near $a_{j}$. 
It is easy to write down the general form of a Fuchsian equation with prescribed singularities and prescribed exponents at the singularities. After a normalization, $n-3$ parameters remain, the so-called accessory parameters. To obtain a conformal metric of curvature 1 , one has to choose these accessory parameters in such a way that the monodromy group of the equation is conjugate to a subgroup of $P S U(2)$.

By a fractional-linear change of the independent variable, one can place one singular point at $\infty$. Then, making changes of the variable $y(z) \mapsto y(z)\left(z-a_{j}\right)^{\beta_{j}}$, one can assume that the smaller exponent at each finite singular point is 0 ; see [40. For the case of four singularities $a_{0}, \ldots, a_{3}$, where $a_{3}=\infty$, we thus obtain Heun's equation in the standard form

$$
y^{\prime \prime}+\left(\sum_{j=0}^{2} \frac{1-\alpha_{j}}{z-a_{j}}\right) y^{\prime}+\frac{A z-\lambda}{\left(z-a_{0}\right)\left(z-a_{1}\right)\left(z-a_{2}\right)} y=0,
$$

where

$$
A=\alpha^{\prime} \alpha^{\prime \prime}, \quad \sum_{j=0}^{2} \alpha_{j}+\alpha^{\prime}+\alpha^{\prime \prime}=2 .
$$

Here the exponents at the singular points are described by the Riemann symbol

$$
P\left\{\begin{array}{ccccc}
a_{0} & a_{1} & a_{2} & \infty & \\
0 & 0 & 0 & \alpha^{\prime \prime} & ; z \\
\alpha_{0} & \alpha_{1} & \alpha_{2} & \alpha^{\prime} &
\end{array}\right\}
$$

The first line lists the singularities, the second the smaller exponents, and the third the larger exponents. So the angle at infinity is $\alpha_{3}=\alpha^{\prime}-\alpha^{\prime \prime}$. The accessory parameter is $\lambda$.

Solving the second equation (2.4) together with $\alpha^{\prime}-\alpha^{\prime \prime}=\alpha_{3}$, we obtain

$$
\alpha^{\prime}=\frac{1}{2}\left(2+\alpha_{3}-\alpha_{0}-\alpha_{1}-\alpha_{2}\right)
$$

and

$$
\alpha^{\prime \prime}=\frac{1}{2}\left(2-\alpha_{3}-\alpha_{0}-\alpha_{1}-\alpha_{2}\right) .
$$

The question of the existence of a spherical quadrilateral with given corners $a_{0}, a_{1}$, $a_{2}, \infty$ and given angles $\pi \alpha_{j}, 0 \leq j \leq 3$, is equivalent to the following: when can one choose real $\lambda$ so that the monodromy group of Heun's equation (2.3) is conjugate to a subgroup of $P S U(2)$ ?

The necessary condition (1.2) can be restated for the equation (2.3) as

$$
\alpha^{\prime \prime}<0 \text {. }
$$

We also have

$$
A=\alpha^{\prime} \alpha^{\prime \prime}=\alpha^{\prime \prime}\left(\alpha_{3}+\alpha^{\prime \prime}\right)
$$

by a simple computation.

One can write (2.3) in several other forms. Assuming that all $a_{j}$ are real, we have the Sturm-Liouville form:

$$
\frac{d}{d x}\left(\prod_{j=0}^{2}\left|x-a_{j}\right|^{1-\alpha_{j}} y^{\prime}\right)+\frac{(A x-\lambda) \operatorname{sgn}\left(\prod_{j=0}^{2}\left(x-a_{j}\right)\right)}{\prod_{j=0}^{2}\left|x-a_{j}\right|^{\alpha_{j}}} y=0 .
$$


Sometimes the Schrödinger form is more convenient:

$$
y^{\prime \prime}-\left(\lambda+\frac{1}{4} \sum_{k=0}^{3} \frac{\alpha_{k}^{2}-1}{x-a_{k}} \prod_{j \neq k}\left(a_{k}-a_{j}\right)\right) \frac{y}{\prod_{j=0}^{3}\left(x-a_{j}\right)}=0,
$$

where all four singularities are in the finite part of the plane. The exponents in the Schrödinger form are $\left(1 \pm \alpha_{j}\right) / 2$. The potential in (2.8) is $F / 2$, where $F$ is the Schwarzian (2.1).

A question similar to our problem was investigated in $23,24,29,45,46$ : when can one choose the accessory parameter so that the monodromy group of Heun's equation preserves a circle? All these authors consider the problem under the assumption

$$
0 \leq \alpha_{j}<1, \quad \text { for } \quad 0 \leq j \leq 3 .
$$

The most comprehensive treatment of this problem is in Smirnov's thesis [45. Smirnov proved that for all sets of data satisfying (2.9), there exists a sequence of values of the accessory parameter $\lambda=\lambda_{k}, k=0, \pm 1, \pm 2, \ldots$ such that the monodromy group of the equation has an invariant circle. Each of the two opposite sides of the corresponding quadrilateral covers a circle $|k|$ times, and the other two sides are proper subsets of their corresponding circles.

The problem of choosing the accessory parameter so that the monodromy group is conjugate to a subgroup in $P S U(2)$ is discussed in 10. However all results of that paper are also proved only under the assumption (2.9).

Assumption (2.9) seems to be essential for the methods of Klein [29], Hilb, Smirnov and Dorfmeister.

\section{ThE CASE $n=4$ WITH TWO INTEGER CORNERS: \\ CONDition ON THE ANGLES}

In the rest of the paper, we study the case $n=4$ with two integers $\alpha_{j}$. We answer the following questions:

a) In the equation (2.3), for which $\alpha_{j}$ can one choose $\lambda$ so that the monodromy group is conjugate to a subgroup of $P S U(2)$ ?

b) If $\alpha_{j}$ satisfy a), how many choices of $\lambda$ are possible?

c) If, in addition, all $a_{j}$ are real, how many choices of real $\lambda$ are possible?

One cannot have exactly one non-integer $\alpha_{j}$. Indeed, in this case the developing map $f$ will have just one branching point on the sphere, which is impossible by the Monodromy Theorem.

Let us consider the case of two non-integers $\alpha_{j}$. In this section we obtain a necessary and sufficient condition on the angles for this case, that is, solve the problem a).

We place the two singularities corresponding to non-integer $\alpha$ at $a_{0}=0$ and $a_{3}=\infty$, and let the total angles at these points be $2 \pi \alpha_{0}$ and $2 \pi \alpha_{3}$, where $\alpha_{0}$ and $\alpha_{3}$ are not integers. Then the developing map has an analytic continuation in $\mathbf{C}^{*}$ from which we conclude that the monodromy group must be a cyclic group generated by a rotation $z \mapsto z e^{2 \pi i \alpha}$, with some $\alpha \in(0,1)$. This means that $f(z)$ is multiplied by $e^{2 \pi i \alpha}$ when $z$ describes a simple loop around the origin. Thus $g(z)=z^{-\alpha} f(z)$ is a single valued function with at most power growth at 0 and $\infty$. Then we have a representation $f(z)=z^{\alpha} g(z)$, where $g$ is a rational function. Then $\alpha_{0}=|k+\alpha|, \alpha_{3}=|j+\alpha|$, where $k$ and $j$ are integers, so either $\alpha_{0}-\alpha_{3}$ or $\alpha_{0}+\alpha_{3}$ is an integer. The angles $2 \pi \alpha_{1}$ and $2 \pi \alpha_{2}$ at the other two singular points $a_{1}$ and 
$a_{2}$ of the metric are integer multiples of $2 \pi$, and they are the critical points of $f$ other than 0 and $\infty$.

Let $g=P / Q$ where $P$ and $Q$ are polynomials without common zeros of degrees $p$ and $q$, respectively. Let $p_{0}$ and $q_{0}$ be the multiplicities of zeros of $P$ and $Q$ at 0 . Then $\min \left\{p_{0}, q_{0}\right\}=0$, because the fraction $P / Q$ is irreducible.

The equation for the critical points of $f$ is the following:

$$
z\left(P^{\prime}(z) Q(z)-P(z) Q^{\prime}(z)\right)+\alpha P(z) Q(z)=0 .
$$

Since $\alpha_{1}$ and $\alpha_{2}$ are integers, we have the following system of equations:

$$
\begin{aligned}
\alpha_{0} & =\left|p_{0}-q_{0}+\alpha\right|, \\
\alpha_{1}+\alpha_{2}-2 & =p+q-\max \left\{p_{0}, q_{0}\right\}, \\
\alpha_{3} & =|p-q+\alpha| .
\end{aligned}
$$

The first and the last equations follow immediately from the representation $f(z)=$ $z^{\alpha} P(z) / Q(z)$ of the developing map. The second equation holds because the lefthand side of (3.1) is a polynomial of degree exactly $p+q$, therefore the sum of the multiplicities of its zeros $a_{1}$ and $a_{2}$ must be $p+q-\max \left\{p_{0}, q_{0}\right\}$.

Solving this system of equations (3.2) in non-negative integers satisfying $\min \left\{p_{0}, q_{0}\right\}=0, p_{0} \leq p, q_{0} \leq q$, we obtain the necessary and sufficient conditions the angles should satisfy, which we state as

Theorem 3.1. Suppose that four points $a_{0}, \ldots, a_{3}$ on the Riemann sphere and numbers $\alpha_{j}>0,0 \leq j \leq 3$, are such that $\alpha_{1}$ and $\alpha_{2}$ are integers $\geq 2$.

The necessary and sufficient conditions for the existence of a metric of curvature 1 on the sphere, with conic singularities at $a_{j}$ and angles $2 \pi \alpha_{j}$ are the following:

a) If $\alpha_{1}+\alpha_{2}+\left[\alpha_{0}\right]+\left[\alpha_{3}\right]$ is even, then $\alpha_{0}-\alpha_{3}$ is an integer, and

$$
\left|\alpha_{0}-\alpha_{3}\right|+2 \leq \alpha_{1}+\alpha_{2} .
$$

b) If $\alpha_{1}+\alpha_{2}+\left[\alpha_{0}\right]+\left[\alpha_{3}\right]$ is odd, then $\alpha_{0}+\alpha_{3}$ is an integer, and

$$
\alpha_{0}+\alpha_{3}+2 \leq \alpha_{1}+\alpha_{2} \text {. }
$$

Sketch of the proof. For a complete proof see [16. Conditions a) and b) are necessary and sufficient for the existence of a unique solution $p, q, p_{0}, q_{0}, \alpha$ of the system (3.2) satisfying

$$
\min \left\{p_{0}, q_{0}\right\}=0, \quad p_{0} \leq p, \quad q_{0} \leq q, \quad \alpha \in(0,1) .
$$

Thus the necessity of these conditions follows from our arguments above.

We may assume without loss of generality that $a_{0}=0, a_{3}=\infty, a_{1}=1$ and $a_{2}=a \in \mathbf{C}$.

Then we set $R(z)=z^{\max \left\{p_{0}, q_{0}\right\}}(z-1)^{\alpha_{1}-1}(z-a)^{\alpha_{2}-1}$. The second equation in (3.2) gives $\operatorname{deg} R=p+q$. Now we consider the equation

$$
z\left(P^{\prime} Q-P Q^{\prime}\right)+\alpha P Q=R .
$$

This equation must be solved in polynomials $P$ and $Q$ of degrees $p$ and $q$ having zeros of multiplicities $p_{0}$ and $q_{0}$ at 0 . Non-zero polynomials of degree at most $p$ modulo proportionality can be identified with the points of the complex projective space $\mathcal{P}^{p}$. The map

$$
W_{\alpha}: \mathcal{P}^{p} \times \mathcal{P}^{q} \rightarrow \mathcal{P}^{p+q}, \quad(P, Q) \mapsto z\left(P^{\prime} Q-P Q^{\prime}\right)+\alpha P Q
$$


is well defined. It is a finite map between compact algebraic varieties, and it can be represented as a linear projection of the Veronese variety. Its degree is equal to the degree

$$
\left(\begin{array}{c}
p+q \\
p
\end{array}\right)
$$

of the Veronese variety. Thus the equation (3.5) always has a complex solution $(P, Q)$. The function $f=z^{\alpha} P / Q$ is then a developing map with the required properties. So conditions a) and b) are sufficient. This completes the proof.

It will be convenient to introduce new parameters instead of $\alpha_{j}$. Besides other advantages, we eliminate the additional parameter $A$ (see (2.3), (2.4)), and the new parameters allow us to treat the cases a) and b) in Theorem 3.1 simultaneously. We will rewrite (2.3) as

$$
z(z-1)(z-a)\left(y^{\prime \prime}-\left(\frac{\sigma}{z}+\frac{m}{z-1}+\frac{n}{z-a}\right) y^{\prime}\right)+\kappa(\sigma+1+m+n-\kappa) z y=\lambda y
$$

where $\kappa$ is an integer, $\sigma \in \mathbf{R}$ is not an integer,

$$
[\sigma] \geq-1 \quad \text { in case a), and }[\sigma]<-1 \text { in case b). }
$$

To achieve this we put $m=\min \left\{\alpha_{1}, \alpha_{2}\right\}-1$, and $n=\max \left\{\alpha_{1}, \alpha_{2}\right\}-1$.

In case a), we set

$$
\sigma=\min \left\{\alpha_{0}, \alpha_{3}\right\}-1
$$

and define $\kappa$ by

$$
2 \kappa=-\left|\alpha_{0}-\alpha_{3}\right|+\alpha_{1}+\alpha_{2}-2 .
$$

In case b), we set

$$
\sigma=-\min \left\{\alpha_{0}, \alpha_{3}\right\}-1
$$

and define $\kappa$ by

$$
2 \kappa=-\alpha_{0}-\alpha_{3}+\alpha_{1}+\alpha_{2}-2 .
$$

In both cases $\kappa$ is an integer because the sums in the right-hand sides of (3.9) and (3.10) are even. Inequalities (3.3) and (3.4) give that $\kappa \geq 0$ in both cases. Since $m+n=\alpha_{1}+\alpha_{2}-2$, we also get

$$
2 \kappa \leq m+n
$$

Furthermore in case b),

$$
\sigma+1 \geq \kappa-(m+n) / 2
$$

because $\alpha_{0}+\alpha_{3} \geq 2 \min \left\{\alpha_{0}, \alpha_{3}\right\}$. Notice that in case a), inequality (3.12) holds trivially because $\sigma+1>0$.

To summarize, the new parameters are three integers $m, n, \kappa$, and one real noninteger number $\sigma$, subject to the conditions (3.12) and

$$
0 \leq m \leq n, \quad 0 \leq 2 \kappa \leq m+n,
$$

Parameters $\alpha_{j}$ are recovered by the formulas

$$
\begin{gathered}
\left(\alpha_{0}, \alpha_{3}\right)=(|\sigma+1|,|m+n+\sigma+1-2 \kappa|), \\
\left(\alpha_{1}, \alpha_{2}\right)=(m+1, n+1),
\end{gathered}
$$

up to a permutation of $\alpha_{1}$ and $\alpha_{2}$, and a permutation of $\alpha_{0}$ and $\alpha_{3}$, and Heun's equation is as (3.7). 


\section{Counting solutions}

In most cases, there is no uniqueness in Theorem 3.1. In this section we determine the number of equivalence classes of metrics for given $a_{j}$ and $\alpha_{j}$, assuming that two of the $\alpha_{j}$ are integers. As explained in the previous section, in this case the Heun equation has a polynomial solution, and a solution of the form $z^{\alpha} P$, where $P$ is a polynomial. We call functions of this last type quasipolynomials.

By substituting a formal power series

$$
H(z)=\sum_{s \in \mathbb{Z}+\beta} h(s) z^{s}
$$

to the equation (3.7), we obtain recurrence relations of the form

$$
c_{s-1} h(s-1)+a_{s} h(s)+b_{s} h(s+1)=0,
$$

which can be visualized as a multiplication of the vector $(h(s))$ by a Jacobi (threediagonal) matrix

$$
\left(\begin{array}{ccccccc} 
& & \ldots & & \ldots & & \\
\ldots & c_{s-1} & a_{s} & b_{s} & 0 & 0 & \ldots \\
\ldots & 0 & c_{s} & a_{s+1} & b_{s+1} & 0 & \ldots \\
\ldots & 0 & 0 & c_{s+1} & a_{s+2} & b_{s+2} & \ldots \\
& & \ldots & & \ldots & &
\end{array}\right) .
$$

The explicit expressions for $a_{s}, b_{s}, c_{s}$ are obtained by substituting (4.1) to (3.7) and equating the coefficients at equal powers of $z$. This yields

$$
\begin{aligned}
b_{s} & =a(s+1)(s-\sigma), \\
a_{s} & =-s((a+1)(s-1-\sigma)-m a-n)-\lambda, \\
c_{s} & =(s-\kappa)(s+\kappa-\sigma-m-n-1) .
\end{aligned}
$$

These expressions can also be found in [40, p. 34, equations (3.3.3a), (3.3.3b), $(3.3 .3 \mathrm{c})]$. We see that an "eigenvector" $h(s)$ can be a finitely supported sequence, say with support $\left[s_{1}, s_{2}\right]$, if and only if $b_{s_{1}-1}=0$ and $c_{s_{2}}=0$. If $b_{s_{1}-1}=0$, and $b_{s} \neq 0$ for $s>s_{1}$, the elements $h(s)$ can be defined recursively from (4.2), with an arbitrary non-zero value of $h\left(s_{1}\right)$. Each $h(s)$ is a polynomial in $\lambda$ of degree $s-s_{1}$, and the condition of termination of the sequence at the place $s_{2}$ is

$$
c_{s_{2}-1} h\left(s_{2}-1\right)+a_{s_{2}} h\left(s_{2}\right)=0 .
$$

This is a polynomial equation of degree $s_{2}-s_{1}+1$ in $\lambda$ which is the condition of having a polynomial solution of degree $d=s_{2}-s_{1}$. A similar condition gives the existence of a quasipolynomial solution. Solutions of (4.7) are eigenvalues of $(d+1) \times(d+1)$ Jacobi matrix obtained by truncating the infinite matrix (4.3) by leaving rows and columns with indexes from $s_{1}$ to $s_{2}$.

Substituting a formal power series

$$
H(z)=\sum_{s=0}^{\infty} h(s)(z-1)^{s}
$$

to the equation (3.7) we obtain another recurrence relation of the form (4.2) with appropriate coefficients $a_{s}, b_{s}$ and $c_{s}$. Since the exponents of (3.7) at the point 1 are 0 and $m+1$, we can always find a holomorphic function $H$ whose power series 
begins with the term $(z-1)^{m+1}$. But to find a power series solution beginning with a constant term, the condition

$$
c_{m-1} h(m-1)+a_{m} h(m)=0
$$

must be satisfied, and this is a polynomial equation of degree $d=m+1$ in $\lambda$. If the condition (4.8) is satisfied, then $h(m+1)$ can be chosen arbitrarily. Equation (4.8) is the triviality condition of the monodromy around 1. Solutions of (4.8) are eigenvalues of the $d \times d$ Jacobi matrix obtained by taking rows and columns of (4.3) with indices from 0 to $m$.

Thus we have four polynomial conditions which are necessary for Heun's equation (3.7) to have two solutions: a polynomial and a quasipolynomial.

(i) $C_{1}(\lambda)=0$ iff there exists a polynomial solution,

(ii) $C_{2}(\lambda)=0$ iff there exists a quasipolynomial solution,

(iii) $C_{3}(\lambda)=0$ iff the monodromy at 1 is trivial, and

(iv) $C_{4}(\lambda)=0$ iff the monodromy at $a$ is trivial.

The degrees of these equations are:

$$
\operatorname{deg} C_{1}=\kappa+1, \quad \operatorname{deg} C_{2}=m+n-\kappa+1, \quad \operatorname{deg} C_{3}=m+1, \quad \operatorname{deg} C_{4}=n+1 .
$$

The first two formulas follow by setting $c_{s_{2}}=0$ in (4.6), and for the other two one has to rewrite (3.7) to place a singular point with integer exponents at 0 , and write the formula for $b(s)$ for this transformed equation (see (4.9) and (5.3) below).

Thus the number of values of $\lambda$ for which all four polynomials $C_{i}$ vanish is at $\operatorname{most} \min \{\kappa+1, m+1, n+1\}$, where we used (3.11). Expressing this in terms of the original exponents $\alpha_{j}$ with the help of (3.9) and (3.10) we obtain

Theorem 4.1. The number of classes of metrics with prescribed angles $2 \pi \alpha_{j}$ at the given points $a_{j}$ is at most

$$
\min \left\{\alpha_{1}, \alpha_{2}, \kappa+1\right\}
$$

where $\kappa$ is defined by (3.9), (3.10),

$$
\kappa+1=\left\{\begin{array}{l}
\left.\left(\alpha_{1}+\alpha_{2}-\left|\alpha_{0}-\alpha_{3}\right|\right) / 2 \quad \text { in case } a\right), \\
\left.\left(\alpha_{1}+\alpha_{2}-\alpha_{0}-\alpha_{3}\right) / 2 \quad \text { in case } b\right) .
\end{array}\right.
$$

We will later see that equality holds for generic $a$. The crucial fact is

Proposition 4.2. Of the four polynomials $C_{j}, 1 \leq j \leq 4$, the polynomial of the smallest degree divides each of the other three polynomials.

Proof. We write Heun's equation in the form (3.7).

1. Suppose that $C_{1}$ is the polynomial of the smallest degree $\kappa+1$. For every root $\lambda$ of $C_{1}$, we have a polynomial solution $p$ of degree at most $\kappa$. So $p$ cannot have a zero of order $\alpha_{1}$ or $\alpha_{2}$, because by assumption these numbers are at least $\kappa+1$. This implies that the monodromy is trivial at 1 and $a$. Then the second solution of Heun's equation also has no singularities at 1 and $a$. So in this case $C_{1}$ divides $C_{2}, C_{3}$ and $C_{4}$.

2. $C_{2}$ cannot be the polynomial of the smallest degree in view of (3.11).

3. It remains to show that if $C_{k}, k \in\{3,4\}$ is the polynomial of the smallest degree and $\lambda$ is a root of $C_{k}$, then $\lambda$ is also a root of $C_{1}$ and $C_{2}$. Then it will follow that there is a polynomial and a quasipolynomial solution, so the monodromy will also be trivial at $a$, that is, $\lambda$ will be a root of all three remaining polynomials. 
Proposition 4.3. Let $m, n, \kappa$ be integers, $0 \leq m \leq n, 0 \leq 2 \kappa \leq m+n$, and $\sigma$ not an integer. Consider the differential equation (3.7) which we write as Dy $=0$, where

$$
D y=z(z-1)(z-a)\left(y^{\prime \prime}-\left(\frac{\sigma}{z}+\frac{m}{z-1}+\frac{n}{z-a}\right) y^{\prime}\right)+\kappa(\sigma+1+\tau) z y-\lambda y,
$$

and $\tau=m+n-\kappa \geq \kappa$. Suppose that $m \leq \kappa$, and that the monodromy at 1 is trivial, that is, all solutions are holomorphic at the point 1 . Then there exist a polynomial solution and a quasipolynomial solution.

Proof. Let us transform our equation (3.7) to the form

$$
\begin{aligned}
& z(z-1)(z-a)\left(y^{\prime \prime}-\left(\frac{m}{z}+\frac{\sigma}{z-1}+\frac{\sigma+2+m+n-2 \kappa}{z-a}\right) y^{\prime}\right) \\
& +\kappa(\kappa-n-1) z y=\lambda y .
\end{aligned}
$$

It has a polynomial solution of degree $k$ simultaneously with the original equation (3.7). Consider the infinite Jacobi matrix (4.3). The triviality of the monodromy of (4.9) at 0 means that $\lambda$ is an eigenvalue of the truncated Jacobi matrix $J_{0}$ given by the first $m+1$ rows and columns. The existence of a polynomial solution of (4.9) of degree $k$ means that $\lambda$ is an eigenvalue of the truncated matrix $J_{1}$ given by the first $k+1$ rows and columns.

By explicit formulas for the entries, the matrix $J_{1}$ is upper block-triangular, the bottom-left $(m+1) \times k$ block being equal to zero, and the top left $(m+1) \times(m+1)$ block of $J_{1}$ equals $J_{0}$. Thus every eigenvalue of $J_{0}$ is an eigenvalue of $J_{1}$.

To show the existence of a quasipolynomial solution $y(z)=z^{\sigma+1} q(z)$, we write the differential equation for $q(\sigma$ will be replaced by $-\sigma-2)$ and then transform it to the form (4.9).

To summarize the contents of this section, we consider, for any given $\alpha_{0}, \ldots, \alpha_{3}$ satisfying conditions a) or b) of Theorem 3.1, the polynomial $F(a, \lambda)$ which is the polynomial of the smallest degree of those $C_{j}$ in Proposition 4.2 . The condition

$$
F(a, \lambda)=0
$$

is equivalent to the statement that the monodromy of Heun's equation is conjugate to a subgroup of $P S U(2)$. Thus equivalence classes of metrics of positive curvature 1 with singularities at $0,1, a, \infty$ with prescribed $\alpha_{j}$ are in one-to-one correspondence with solutions of the equation (4.10).

Remark. The value $\lambda$ in (4.10) depends not only on the quadrilateral (or a metric) that we consider but also on the choice of the Heun equation. Different Heun equations corresponding to the same quadrilateral can be obtained by cyclic permutation of the vertices, and by the different choices of exponents at the singularities. The angle at a vertex only fixes the absolute value of the difference of the exponents. The values of $\lambda$ corresponding to the same quadrilateral but different Heun equations are related by fractional-linear transformations.

\section{Counting Real solutions}

In this section we assume that $a$ is real and estimate from below the number of real Heun's equations with given conic singularities at $0,1, a, \infty$ with prescribed angles and unitary monodromy, or, which is the same, the number of real solutions 
$\lambda$ of equation (4.10). We will also show that for generic $a$ we have equality in the inequality of Theorem 4.1 for the number of complex solutions.

Our estimates will be based on the following lemma.

Lemma 5.1. Let $J$ be a real $(d+1) \times(d+1)$ Jacobi matrix

$$
J=\left(\begin{array}{cccccccc}
a_{0} & b_{0} & 0 & 0 & \ldots & 0 & 0 & 0 \\
c_{0} & a_{1} & b_{1} & 0 & \ldots & 0 & 0 & 0 \\
0 & c_{1} & a_{2} & b_{2} & \ldots & 0 & 0 & 0 \\
\ldots & \ldots & \ldots & \ldots & \ldots & \ldots & \ldots & \ldots \\
\ldots & \ldots & \ldots & \ldots & \ldots & \ldots & \ldots & \ldots \\
0 & 0 & 0 & 0 & \ldots & c_{d-2} & a_{d-1} & b_{d-1} \\
0 & 0 & 0 & 0 & \ldots & 0 & c_{d-1} & a_{d}
\end{array}\right) .
$$

a) If $b_{j} c_{j}>0,0 \leq j \leq d-1$, then all eigenvalues of $J$ are real and simple.

b) If $c_{j} \neq 0$ for $0 \leq j \leq d-1$, then we have

$$
J^{T} R=R J,
$$

where $R=\operatorname{diag}\left(r_{0}, \ldots, r_{d}\right), r_{0}=1$ and

$$
r_{j}=r_{j-1} \frac{b_{j-1}}{c_{j-1}}, \quad 1 \leq j \leq d .
$$

c) Suppose that the sequence $d_{j}=b_{j} c_{j}$ has the property $d_{j}>0$ for $0 \leq j \leq k$ and $d_{j}<0$ for $k+1 \leq j \leq d$. Then the number of pairs of non-real eigenvalues, counting multiplicity, is at most $[(d-k) / 2]$.

Proof. Statement a) in contained in [20; we include a simple proof for convenience.

Consider the matrix $S=\operatorname{diag}\left(s_{0}, \ldots, s_{d}\right), s_{0}=1$, and

$$
s_{j}=s_{j-1} \sqrt{b_{j-1} / c_{j-1}}, \quad 1 \leq j \leq d .
$$

Under the assumption of part a), the fraction under the square root is positive, and the matrix $S$ is the positive square root of the matrix $R$ given in part b), $S^{2}=R$. By explicit calculation, the matrix

$$
\tilde{J}=S J S^{-1}
$$

is real and symmetric, so it is diagonalizable and has real eigenvalues. The top right $d \times d$ submatrix of $\tilde{J}-\lambda I$, where $I$ is the identity matrix and $\lambda$ is an eigenvalue of $\tilde{J}$, is lower triangular and has the determinant

$$
\sqrt{b_{0} \ldots b_{d-1} c_{0} \ldots c_{d-1}} \neq 0 .
$$

Hence all eigenvalues of $\tilde{J}$ are simple.

Statement b) is proved by direct calculation.

To prove statement c), we notice that our assumption about signs of $d_{j}$ implies that there are $[(d-k) / 2]$ negative numbers among $r_{1}, \ldots, r_{n}$, and the rest are positive. Condition (5.1) means that our matrix $J$ is symmetric with respect to the bilinear form $(x, y)_{R}=x^{T} R y$, that is,

$$
(J x, y)_{R}=(x, J y)_{R} .
$$

Quadratic form $(x, x)_{R}$ has $[(d-k) / 2]$ negative squares, so our matrix $J$ has at most $2[(d-k) / 2]$ non-real eigenvalues, counted with algebraic multiplicities, according to the theorem of Pontrjagin [38]. This proves the lemma. 
We immediately obtain the following

Proposition 5.2. When the parameter $a$ in (3.7) is real and is sufficiently close to 0 or 1 , then all metrics in Theorem 4.1 are symmetric with respect to the real line, and thus correspond to spherical quadrilaterals.

Proof. When $a$ is real, the matrix $J$ is real. When $a \rightarrow 0$, it follows from the formulas (4.4) - (4.6) that $J$ tends to a lower triangular matrix with distinct elements on the main diagonal. All eigenvalues of such a matrix are real and distinct. So for small enough $a>0$ it also has real distinct eigenvalues. To apply the same argument when $a$ is real and $a \rightarrow 1-$, we make the change of the independent variable $z \mapsto 1-z$ in (3.7).

Our main result on the symmetric case is the following:

Theorem 5.3. Consider the metrics of curvature 1 on the sphere with real conic singularities $a_{0}, a_{1}, a_{2}, a_{3}$ and the corresponding angles $2 \pi \alpha_{0}, 2 \pi \alpha_{1}, 2 \pi \alpha_{2}, 2 \pi \alpha_{3}$, where $\alpha_{1}$ and $\alpha_{2}$ are integers. Suppose that conditions of Theorem 3.1 are satisfied. Then

(i) If the pairs $\left(a_{0}, a_{3}\right)$ and $\left(a_{1}, a_{2}\right)$ do not separate each other on the circle $\overline{\mathbf{R}}$, then all metrics with these angles and singularities are symmetric. Their number is equal to

$$
\min \left\{\alpha_{1}, \alpha_{2}, \kappa+1\right\}
$$

where $\kappa$ is defined in (3.9) and (3.10).

(ii) If the pairs $\left(a_{0}, a_{3}\right)$ and $\left(a_{1}, a_{2}\right)$ separate each other, then the number of classes of symmetric metrics is at least

$$
\min \left\{\alpha_{1}, \alpha_{2}, \kappa+1\right\}-2\left[\frac{1}{2} \min \left\{\alpha_{1}, \alpha_{2}, \delta\right\}\right],
$$

where

$$
\delta=\frac{1}{2} \max \left\{\alpha_{1}+\alpha_{2}-\left[\alpha_{0}\right]-\left[\alpha_{3}\right], 0\right\} .
$$

(iii) There is an $\epsilon>0$ depending on the $\alpha_{j}$ such that if

$$
\left|\frac{\left(a_{2}-a_{0}\right)\left(a_{3}-a_{1}\right)}{\left(a_{1}-a_{0}\right)\left(a_{3}-a_{2}\right)}\right|<\epsilon
$$

in (2.3), then all of the metrics are symmetric, and their number is as in (i).

The expression under the absolute value in the left-hand side of (5.2) is the cross-ratio which is equal to $a$ when the vertices are $0,1, a, \infty$.

In section 16 we will show that the estimate in (ii) is achieved sometimes, and in section 15 we give another independent proof of this estimate.

Corollary 5.4. Suppose that $a_{j}$ are real. If the pairs $\left(a_{0}, a_{3}\right)$ and $\left(a_{1}, a_{2}\right)$ separate each other on $\overline{\mathbf{R}}$, and

$$
\left[\alpha_{0}\right]+\left[\alpha_{3}\right]+2 \geq \alpha_{1}+\alpha_{2}
$$

then all metrics with singularities at $a_{j}$ and angles $\pi \alpha_{j}$ are symmetric with respect to the real line.

If case a) of Theorem 3.1 prevails, this corollary can also be obtained as a special case of Theorem 5.2 from 32 . 
Proof of Theorem 5.3. Let us transform our equation (3.7) to the form (4.9). This equation has the same exponents at the singularities as (3.7), but we placed the point with the smaller integer exponent $m$ at 0 . The recurrence relations similar to (4.2) have in this case the following coefficients:

$$
\begin{aligned}
c_{s} & =(\kappa-s)(\kappa-n-s-1), \\
a_{s} & =-s(s+\sigma+1+n-2 \kappa+a(s-1-m-\sigma)) \\
b_{s} & =a(s+1)(s-m) .
\end{aligned}
$$

So

$$
b_{s} c_{s}=(\kappa-s)(\kappa-n-s-1) a(s+1)(s-m)
$$

which is positive for $a>0$ and $0 \leq s<\min \{\kappa, m\}$. Thus for $a>0$ all eigenvalues are real and distinct by Lemma 5.1 a) with $d=\min \{m, \kappa\}+1$. This proves (i).

For the case (ii), that is, $a<0$, we transform equation (4.9) by the change of the variable $z^{\prime}=1-z$ into equation

$$
\begin{aligned}
& z(z-1)\left(z-a^{\prime}\right)\left(y^{\prime \prime}-\left(\frac{\sigma}{z}+\frac{m}{z-1}+\frac{\sigma+m+n+2-2 \kappa}{z-a^{\prime}}\right) y^{\prime}\right) \\
& +\kappa(\kappa-n-1) z y=\lambda y .
\end{aligned}
$$

Here $a^{\prime}=1-a$. The coefficients of the recurrence become

$$
\begin{aligned}
c_{s} & =(\kappa-s)(\kappa-n-s-1) \\
a_{s} & \left.=-s(s+m+n+1-2 \kappa)+a^{\prime}(s-m-\sigma-1)\right), \\
b_{s} & =a^{\prime}(s+1)(s-\sigma) .
\end{aligned}
$$

So we have

$$
c_{s} b_{s}=(\kappa-s)(\kappa-n-s-1) a^{\prime}(s+1)(s-\sigma),
$$

which is positive when $a^{\prime}>0, \sigma+1>\kappa$, and $0 \leq s<\kappa$. Thus under this condition, all eigenvalues are real. The range $a<0$ is covered by $a^{\prime}>0$.

If $\sigma+1 \leq \kappa$, then the Jacobi matrix with entries (5.6) has the property described in Lemma $[5.1 \mathrm{c})$, where $R$ has $[\sigma]+1$ positive squares and $[(\kappa-[\sigma]) / 2]$ negative squares. So the number of pairs of non-real eigenvalues, counting algebraic multiplicities, is at most $(\kappa-[\sigma]) / 2$. We recall that $\kappa+1$ is the degree of the polynomial $C_{1}$ in Proposition 4.2. So the polynomial of minimum degree among the $C_{j}$ has at most $(1 / 2) \min \{\kappa+1, m+1, \kappa-[\sigma]\}$ pairs of non-real zeros, and using the value of $\kappa$ from (3.9), (3.10) and the inequality (3.8), we obtain (ii).

Statement (iii) is an immediate consequence of Proposition 5.2 .

\section{IntRoduCtion to NETS}

In this section we begin a different treatment of spherical polygons which is independent of sections 25 .

Definition 6.1. A spherical $n$-gon $Q$ is marked if one of its corners, labeled $a_{0}$, is identified as the first corner, and the other corners are labeled so that $a_{0}, \ldots, a_{n-1}$ are in the counterclockwise order on the boundary of $Q$.

We call $Q$ a spherical polygon when $n$ is not specified. When $n=2,3$ and 4 , we call $Q$ a spherical digon, triangle and quadrilateral, respectively. For $n=1$, there is a unique marked 1-gon with the angle $\pi$ at its single corner. For convenience, we often drop "spherical" and refer simply to $n$-gons, polygons, etc. 
Let $Q$ be a marked spherical polygon and $f: Q \rightarrow \mathbf{S}$ its developing map. The images of the sides $\left(a_{j}, a_{j+1}\right)$ of $Q$ are contained in geodesics (great circles) on $\mathbf{S}$. These geodesics define a partition $\mathcal{P}$ of $\mathbf{S}$ into vertices (intersection points of the circles), edges (arcs of circles between the vertices) and faces (components of the complement to the circles). Some corners of $Q$ may be integer (i.e., with angles $\pi \alpha$ where $\alpha$ is an integer). Two sides of $Q$ meeting at its integer corner are mapped by $f$ into the same circle.

The corners of $Q$ with integer (resp., non-integer) angles are called its integer (resp., non-integer) corners. The order of a corner is the integer part of its angle. A removable corner is an integer corner of order 1 . A polygon $Q$ with a removable corner is isometric to a polygon with a smaller number of corners.

A polygon with all integer corners is called rational. All sides of a rational polygon map to the same circle.

Definition 6.2. The preimage of $\mathcal{P}$ defines a cell decomposition $\mathcal{Q}$ of $Q$, called the net of $Q$. The corners of $Q$ are vertices of $\mathcal{Q}$. In addition, $\mathcal{Q}$ may have side vertices and interior vertices. If the circles of $\mathcal{P}$ are in general position, interior vertices have degree 4 , and side vertices have degree 3. Each face $F$ of $\mathcal{Q}$ maps one-to-one onto a face of $\mathcal{P}$. An edge $e$ of $\mathcal{Q}$ maps either onto an edge of $\mathcal{P}$ or onto a part of an edge of $\mathcal{P}$. The latter possibility may happen when $e$ has an end at an integer corner of $Q$. The adjacency relations of the cells of $\mathcal{Q}$ are compatible with the adjacency relations of their images in $\mathbf{S}$. The net $\mathcal{Q}$ is completely defined by its 1-skeleton, a connected planar graph. When it does not lead to confusion, we use the same notation $\mathcal{Q}$ for that graph.

If $C$ is a circle of $\mathcal{P}$, then the intersection $\mathcal{Q}_{C}$ of $\mathcal{Q}$ with the preimage of $C$ is called the $C$-net of $Q$. Note that the intersection points of $\mathcal{Q}_{C}$ with preimages of other circles of $\mathcal{P}$ are vertices of $\mathcal{Q}_{C}$. A $C$-arc of $Q$ (or simply an arc when $C$ is not specified) is a non-trivial path $\gamma$ in the 1-skeleton of $\mathcal{Q}_{C}$ that may have a corner of $Q$ only as its endpoint. If $\gamma$ is a subset of a side of $Q$, then it is a boundary arc. Otherwise, it is an interior arc. The order of an arc is the number of edges of $\mathcal{Q}$ in it. An arc is maximal if it is not contained in a larger arc. Each side $L$ of $\mathcal{Q}$ is a maximal boundary arc. The order of $L$ is, accordingly, the number of edges of $\mathcal{Q}$ in $L$.

Examples of nets are shown in Figures 2, 4, 6, 8, 9, The net of a quadrilateral $Q$ in Figure 2 has six vertices, two of them interior, ten edges, six of them interior, and two maximal interior arcs (which are loops). The net of a triangle $T_{2}$ in Figure 4 has five vertices, two of them side vertices, and seven edges, two of them interior.

Definition 6.3. We say that $Q$ is reducible if its net has an interior arc with endpoints at two distinct corners of $Q$. Such an arc partitions $Q$ into two proper sub-polygons. Otherwise, $Q$ is irreducible. We say that $Q$ is primitive if it is irreducible and its net does not contain an interior arc that is a loop.

Examples of primitive quadrilaterals are shown in Figures 6, 8, 9, The quadrilateral in Figure 2 is irreducible but not primitive. The quadrilaterals in Figures 12 and 13 are reducible.

Definition 6.4. Two polygons $Q$ and $Q^{\prime}$ are strongly combinatorially equivalent if there is an orientation-preserving homeomorphism $h: Q \rightarrow Q^{\prime}$ mapping the corners of $Q$ to the corners of $Q^{\prime}$, and the net $\mathcal{Q}$ of $Q$ to the net $\mathcal{Q}^{\prime}$ of $Q^{\prime}$. For marked 
polygons $Q$ and $Q^{\prime}$, we also require that the marked corner $a_{0}$ of $Q$ is mapped by $h$ to the marked corner $a_{0}^{\prime}$ of $Q^{\prime}$.

Thus a strong equivalence class of spherical polygons is a combinatorial object. It is completely determined by the labeling of the corners and the adjacency relations. We'll call such an equivalence class "a net" when this would not lead to confusion.

Conversely, given labeling of the corners and a partition $\mathcal{Q}$ of a disk with the adjacency relations compatible with the adjacency relations of $\mathcal{P}$, a spherical polygon with the net $\mathcal{Q}$ can be constructed by gluing together the cells of $\mathcal{P}$ according to the adjacency relations of $\mathcal{Q}$. Such a polygon is unique (up to an isometry preserving the labels of the corners) if the image of $a_{0}$, the direction in which the image of the edge $\left(a_{0}, a_{1}\right)$ is traversed, and the images of integer vertices which are different from the vertices of $\mathcal{P}$, are fixed.

If a polygon $Q$ is not irreducible, Definition 6.4 is not convenient. For example, quadrilaterals in Figures $12 \mathrm{a}-\mathrm{c}$ are not strongly equivalent, yet each of them is obtained by gluing a triangle $T_{0}$ and a disk $\nabla$ along their common side. To reduce the number of cases in classification, we adopt a weaker equivalence relation, defined inductively as follows.

Definition 6.5. Two irreducible polygons $Q$ and $Q^{\prime}$ are combinatorially equivalent if they are strongly combinatorially equivalent.

Two rational polygons $Q$ and $Q^{\prime}$ with all sides mapped to the same circle $C$ of $\mathcal{P}$ are combinatorially equivalent if there is an orientation-preserving homeomorphism $Q \rightarrow Q^{\prime}$ mapping the net $\mathcal{Q}_{C}$ of $Q$ to the net $\mathcal{Q}_{C}^{\prime}$ of $Q^{\prime}$.

If $Q$ and $Q^{\prime}$ are reducible, each of them represented as the union of two polygons $Q_{0}$ and $Q_{1}$ (resp., $Q_{0}^{\prime}$ and $Q_{1}^{\prime}$ ) glued together along their common side, then $Q$ and $Q^{\prime}$ are combinatorially equivalent when there is an orientation-preserving homeomorphism $h: Q \rightarrow Q^{\prime}$ inducing combinatorial equivalence between $Q_{0}$ and $Q_{0}^{\prime}$, and between $Q_{1}$ and $Q_{1}^{\prime}$.

In what follows we classify all equivalence classes of nets in the case when $\mathcal{P}$ is defined by two circles. In this case, the boundary of each 2-cell of the net $\mathcal{Q}$ of $Q$ consists of two segments mapped to the arcs of distinct circles, with the vertices at the common endpoints of the two segments and, possibly, at some integer corners of $Q$.

By the Uniformization Theorem, each marked spherical polygon is conformally equivalent to a closed disk with marked points on the boundary. In the case of a quadrilateral, we have four marked points, so conformal class of a quadrilateral depends on one parameter, the modulus of the quadrilateral. In section 14 below we will study whether for given permitted angles of a quadrilateral an arbitrary modulus can be achieved. This will be done by the method of continuity, and for this we'll need some facts about deformation of spherical quadrilaterals (see section 13).

\section{NETS FOR A TWO-CIRCLE PARTITION}

Let us consider a partition $\mathcal{P}$ of the Riemann sphere $\mathbf{S}$ by two transversal circles intersecting at the angle $\alpha$ (see Figure1). Vertices $N$ and $S$ of $\mathcal{P}$ are the intersection points of the two circles.

We measure the angles in multiples of $\pi$, so that $0<\alpha<1$, and the complementary to $\alpha$ angle is $\beta=1-\alpha$. An angle that is an integer multiple of $\pi$ is called, 


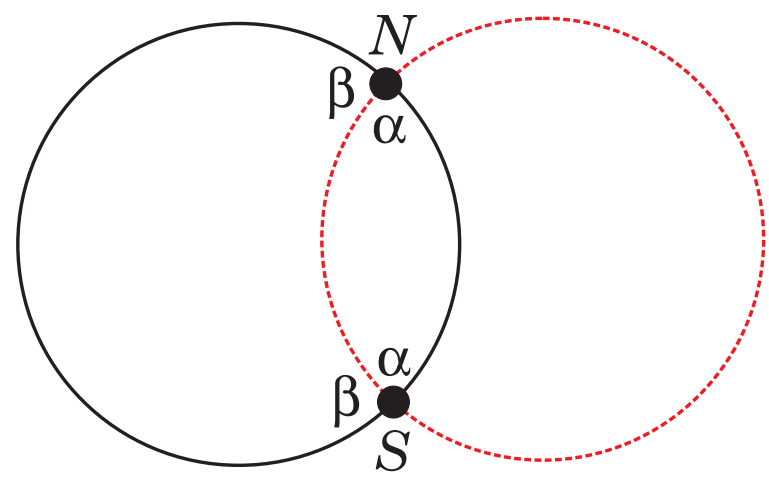

Figure 1. Partition $\mathcal{P}$ of the Riemann sphere by two circles.

accordingly, an integer angle. A corner with an integer angle is called an integer corner.

Let $Q$ be a spherical $n$-gon over $\mathcal{P}$ (see Definition 1.1). We assume $Q$ to be a marked polygon (see Definition 6.1).

Theorem 7.1. An irreducible spherical polygon $Q$ over the partition $\mathcal{P}$ has at most two non-integer corners.

Proof. We prove this statement by induction on the number $m$ of faces of the net $\mathcal{Q}$ of $Q$. If $m=1$, then $Q$ maps one-to-one to a face of $\mathcal{P}$, thus it has exactly two non-integer corners.

Let $m>1$. Suppose first that $\mathcal{Q}$ has a maximal interior $\operatorname{arc} \gamma$ that is not a loop. Let $p$ and $q$ be the endpoints of $\gamma$. Since $\gamma$ is maximal, both $p$ and $q$ are at the boundary of $Q$. Since $Q$ is irreducible, at least one of them, say $p$, is not a corner of $Q$. Thus $\gamma$ partitions $Q$ into two polygons, $Q^{\prime}$ and $Q^{\prime \prime}$, each of them having less than $m$ faces of its net. The induction hypothesis applied to $Q^{\prime}$ and $Q^{\prime \prime}$ implies that each of them has at most two non-integer corners. But the corners of $Q^{\prime}$ and $Q^{\prime \prime}$ at $p$ are non-integer, while $Q$ does not have a corner at $p$. Thus $Q$ has at most two non-integer corners.

Consider now the case when all maximal interior arcs of $\mathcal{Q}$ are loops. Since the 1 -skeleton of $\mathcal{Q}$ is connected, there exists a maximal interior arc $\gamma$ of $\mathcal{Q}$ with both ends at a corner $p$ of $\mathcal{Q}$. The disk $D$ bounded by $\gamma$ maps one-to-one to the disk in $\mathbf{S}$ bounded by the circle $C$ of $\mathcal{P}$ to which $\gamma$ is mapped. Let $C^{\prime}$ be the other circle of $\mathcal{P}$. Then $\gamma$ intersects with $\mathcal{Q}_{C^{\prime}}$ at exactly two points. Let $\gamma^{\prime}$ be the maximal interior arc of $\mathcal{Q}_{C^{\prime}}$ intersecting $\gamma$ at those two points. By assumption, $\gamma^{\prime}$ is a loop. If one of those points is $p$, then $p$ is a preimage of a vertex of $\mathcal{P}$, and $\gamma^{\prime}$ has a single intersection point $q$ with $\gamma$ inside $Q$. But this is impossible because the complement to the union of the disks bounded by $\gamma$ and $\gamma^{\prime}$ would contain a face of $\mathcal{Q}$ whose boundary would not be a circle.

Thus $p$ is not a preimage of a vertex of $\mathcal{P}$, and both intersection points $q$ and $q^{\prime}$ of $\gamma$ and $\gamma^{\prime}$ are interior vertices of $\mathcal{Q}$. Then $\gamma^{\prime}$ must contain a corner $p^{\prime}$ of $\mathcal{Q}$. Otherwise the complement to the union of the disks bounded by $\gamma$ and $\gamma^{\prime}$ would contain a face of $\mathcal{Q}$ whose boundary would not be a circle. The same arguments as above imply that $p^{\prime}$ is not a preimage of a vertex of $\mathcal{P}$, thus the union of $\gamma$ and $\gamma^{\prime}$ is a pseudo-diagonal of $Q$ shown in Figure 2, Removing these two loops, we obtain a 


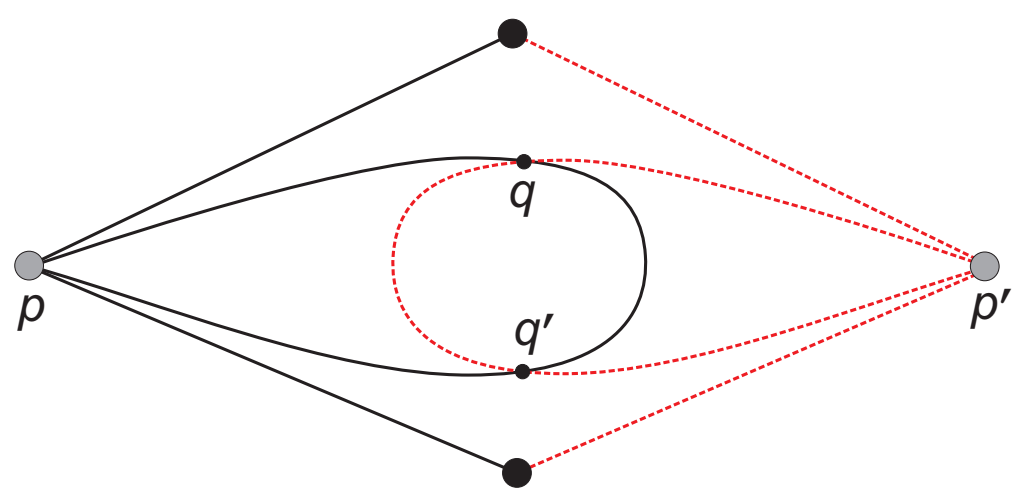

Figure 2. Pseudo-diagonal connecting two integer corners of $Q$.

polygon having $m-4$ faces in its net, with the same number of non-integer corners as $Q$. By the induction hypothesis, $Q$ must have at most two non-integer corners. This completes the proof of Theorem 7.1

Rational spherical polygons. All corners of a rational polygon $Q$ are integer, and all its sides map to the same circle $C$ of $\mathcal{P}$. Thus $Q$ is completely determined, up to combinatorial equivalence, by its $C$-net $\mathcal{Q}_{C}$. Each maximal arc of $\mathcal{Q}_{C}$ connects two corners of $Q$. If $Q$ is irreducible, $\mathcal{Q}_{C}$ does not have interior arcs.

\section{PRimitive SPHERICAL POLYGONS With TWO NON-INTEGER CORNERS}

In this section, $Q$ denotes a marked primitive spherical $n$-gon with two noninteger corners over the partition $\mathcal{P}$, one of these two corners labeled $a_{0}$, and the other one labeled $a_{k}$ where $0<k<n$. The sides of $Q$ are labeled $L_{j}$ so that $a_{j-1}$ and $a_{j}$ are the ends of $L_{j}$, with $a_{n}$ identified with $a_{0}$. We assume that the sides $L_{j}$ for $1 \leq j \leq k$ belong to the preimage of a circle $C$ of $\mathcal{P}$, while the sides $L_{j}$ for $k<j \leq n$ belong to the preimage of the circle $C^{\prime} \neq C$ of $\mathcal{P}$.

Lemma 8.1. The net $\mathcal{Q}$ of $Q$ does not have interior vertices.

Proof. Let $q$ be an interior vertex of $\mathcal{Q}$, and let $F$ be a face of $\mathcal{Q}$ adjacent to $q$. Let $\gamma$ be a maximal arc of $\mathcal{Q}_{C}$ through $q$, where $C$ is a circle of $\mathcal{P}$. Since $\gamma$ is an interior maximal arc, it may end either at a corner of $Q$ or at a side. Since $Q$ is primitive, $\gamma$ is not a loop, and the ends of $\gamma$ cannot be at two distinct corners of $Q$. If an end $p$ of $\gamma$ is at a side of $Q$, let $L$ and $\gamma^{\prime}$ be the maximal $\operatorname{arcs}$ of $\mathcal{Q}_{C^{\prime}}$, where $C^{\prime} \neq C$, passing through $p$ and $q$, respectively. Note that $L$ is a side of $Q$ while $\gamma^{\prime}$ is an interior arc. We may assume that there are two adjacent faces, $F$ and $F^{\prime}$, of $\mathcal{Q}$ having a common segment $p q$ of $\gamma$ in their boundary. If there are no such faces, then we can replace $q$ by an interior vertex of $\mathcal{Q}$ on $\gamma$ closest to $p$. Then each of these two faces must have an integer corner in its boundary where $L$ and $\gamma^{\prime}$ intersect, otherwise the intersection of its boundary with $\mathcal{Q}_{C}$ would not be connected. The two corners must be distinct, since they are the ends of a side $L$ of $Q$. This implies that an interior arc $\gamma^{\prime}$ has its ends at two distinct corners of $Q$, thus $Q$ is not irreducible. 
Corollary 8.2. Each interior arc of the net $\mathcal{Q}$ of $Q$ is maximal and has order 1.

Lemma 8.3. Each of the two non-integer corners of $Q$ has order 0 .

Proof. Let $p$ be a non-integer corner of $Q$ of order greater than 0 . Since $p$ is mapped to a vertex of $\mathcal{P}$, there is a face $F$ of the net $\mathcal{Q}$ of $Q$ having $p$ as its vertex, with two interior arcs, $\gamma$ and $\gamma^{\prime}$, adjacent to $p$ in its boundary. The arcs $\gamma$ and $\gamma^{\prime}$ belong to preimages of two different circles of $\mathcal{P}$. The other ends of $\gamma$ and $\gamma^{\prime}$ cannot be corners of $Q$, thus they must be side vertices of $\mathcal{Q}$. This implies that the preimage of each of the two circles of $\mathcal{P}$ in the boundary of $F$ is not connected, a contradiction.

Corollary 8.4. Any interior arc of $\mathcal{Q}$ has one of its ends at an integer corner $a_{i}$ of $Q$ and another end on the side $L_{j}$, where either $0<i<k<j \leq n$ or $0<j \leq k<i<n$.

Definition 8.5. Let $Q$ be a marked primitive $n$-gon with two non-integer corners labeled $a_{0}$ and $a_{k}$, and let $\mathcal{Q}$ be the net of $Q$. For each pair $(i, j)$, let $\mu(i, j)$ be the number of interior arcs of $\mathcal{Q}$ with one end at the integer corner $a_{i}$ and the other end on the side $L_{j}$. Note that $\mu(i, j)$ may be positive only when either $0<i<k<j \leq n$ or $0<j \leq k<i<n$, due to Corollary 8.4. We call the set $\mathcal{T}$ of the pairs $(i, j)$ for which $\mu(i, j)>0$ is the $(n, k)$-type of $Q$ (or simply the type of $Q$ when $n$ and $k$ are fixed), and the numbers $\mu(i, j)$ the multiplicities. We'll see in Lemma 8.6 below that the number of pairs in an $(n, k)$-type is at most $n-2$. An $(n, k)$-type with exactly $n-2$ pairs is called maximal.

Since interior $\operatorname{arcs}$ of $\mathcal{Q}$ do not intersect inside $Q$, the $(n, k)$-type of $Q$ cannot contain two pairs $\left(i_{0}, j_{0}\right)$ and $\left(i_{1}, j_{1}\right)$ satisfying any of the following four conditions:

$$
\begin{aligned}
& i_{0}<i_{1}<k<j_{0}<j_{1}, \\
& j_{0}<j_{1} \leq k<i_{0}<i_{1}, \\
& i_{0}<j_{1} \leq k<j_{0}<i_{1}, \\
& j_{1} \leq i_{0}<k<i_{1}<j_{0} .
\end{aligned}
$$

Interior arcs of $\mathcal{Q}$ can be canonically ordered, starting from the arc closest to the marked corner $a_{0}$, so that any two consecutive arcs belong to the boundary of a cell of $\mathcal{Q}$. The linear order on the interior $\operatorname{arcs}$ of $\mathcal{Q}$ induces interior order on the pairs $(i, j)$ in the type $\mathcal{T}$ of $Q$.

Lemma 8.6. The number of pairs in the $(n, k)$-type $\mathcal{T}$ of $Q$ is at most $n-2$.

Proof. Let $i, j \in \mathcal{T}$ be the first pair, corresponding to the interior arcs of $\mathcal{Q}$ closest to $a_{0}$. We may assume that $1 \leq i<k$ and $n-k<j \leq n$. Otherwise, we exchange $k$ and $n-k$. Let $(i, m) \in \mathcal{T}$ be the pair furthest from $a_{0}$, with the same $i$ as the first pair. Then there are at most $n-m+1$ pairs in $\mathcal{T}$ with the same index $i$. The last arc of $\mathcal{Q}$ with the ends at the vertex $a_{i}$ and the side $L_{m}$ partitions $Q$ into two polygons, $Q^{\prime}$ and $Q^{\prime \prime}$, with $Q^{\prime \prime}$ containing $a_{0}$. Contracting $Q^{\prime \prime}$ to a point, we obtain an $(m-i)$-gon $\tilde{Q}$ with the $(m-i, k-i)$-type $\tilde{\mathcal{T}}$ obtained from $\mathcal{T}$ by deleting all pairs with the same $i$ as the first one, and relabeling vertices and sides. Since $m \leq n$ and $i>0$, we may assume inductively that the type $\tilde{\mathcal{T}}$ of $\tilde{Q}$ has at most $m-i-2$ pairs. This implies that the type $\mathcal{T}$ has at most $n-i-1 \leq n-2$ pairs. 
Lemma 8.7. Any $(n, k)$-type can be obtained from a (non-unique) maximal $(n, k)$ type if some of the multiplicities are permitted to be zero.

Proof. Let $\mathcal{T}$ be an $(n, k)$-type with less than $n-2$ pairs. We want to show that one can add a pair to $\mathcal{T}$. We prove it by induction on $n$, the case $n=2$ being trivial. We use notation of the proof of Lemma 8.6. Note first that, if $i>1$, then a pair $(1, n)$ can be added to $\mathcal{T}$. Thus we may assume that $i=1$. Next, $\mathcal{T}$ should contain all $n-m+1$ pairs $(1, m), \ldots,(1, n)$, otherwise a missing pair can be added to $\mathcal{T}$. Finally, we can assume inductively that $\mathcal{T}^{\prime}$ contains exactly $m-i-2=m-3$ pairs. Thus the number of pairs in $\mathcal{T}$ should be $(n-m+1)+(m-3)=n-2$.

Proposition 8.8. For given $n \geq 2$ and $k, 0<k<n$, the number $M(n, k)$ of distinct maximal $(n, k)$-types satisfies the following recurrence:

$$
M(n, k)=\sum_{m=1}^{k} M(n-m, k-m+1)+\sum_{m=1}^{n-k} M(n-m, n-k-m+1) .
$$

Since $M(2,1)=1$, this implies that

$$
\sum_{k, l=1}^{\infty} M(k+l, k) x^{k} y^{l}=\frac{(1-x)(1-y)}{(1-x)(1-y)-x(1-y)-y(1-x)} .
$$

Proof. This recurrence follows from construction in the proof of Lemma 8.6 .

The following classification is an immediate consequence of Definition 8.5

Theorem 8.9. For any $n \geq 2$, any $k, 0<k<n$, any set $\mathcal{T}$ of pairs $(i, j)$ with either $0<i<k<j \leq n$ or $0<j \leq k<i<n$, such that no two pairs $\left(i_{0}, j_{0}\right)$ and $\left(i_{1}, j_{1}\right)$ in $\mathcal{T}$ satisfy any of the conditions (8.1) $a$ - $d$, and any positive integers $\nu(i, j),(i, j) \in \mathcal{T}$, there exists a marked primitive spherical $n$-gon, unique up to combinatorial equivalence, with two non-integer corners, one of them marked, with the type $\mathcal{T}$ and multiplicities $\nu(i, j)$.

We are going to apply Theorem 8.9 to classification of spherical quadrilaterals. In this section, we consider primitive quadrilaterals (Corollaries 8.12 and 8.13 ), and in the next section irreducible (but not necessarily primitive) quadrilaterals (Theorem 9.5 and Corollary 9.6). We also classify spherical digons and triangles (Corollaries 8.10 and 8.11), used below as building blocks for constructing reducible quadrilaterals.

Corollary 8.10. Each primitive digon ( $n$-gon for $n=2$ ) with two non-integer corners maps one-to-one to a face of $\mathcal{P}$, with its two corners mapped to distinct vertices of $\mathcal{P}$. There is a single (empty) $(2,1)$-type of a primitive digon.

Let $X$ (see Figure 3) be a point on a circle of $\mathcal{P}$ shown by a solid line, inside a disk $\mathbf{D}$ bounded by the circle $C$ of $\mathcal{P}$ shown by a dashed line. For $\mu \geq 0$, let $T_{\mu}$ (see Figure 4) be a primitive triangle having a non-integer corner $a_{0}$ mapping to $N$, a non-integer corner $a_{1}$ mapped to $S$ (resp., to $N$ ) when $\mu$ is even (resp., odd), and an integer corner $a_{2}$ of order $\mu+1$ mapped to $X$. The small black dots in Figure 4 indicate the preimages of the vertices of $\mathcal{P}$ which are not corners of $T_{\mu}$ (though they are vertices of its net). The angle at the corner $a_{1}$ of $T_{\mu}$ is equal (resp., complementary) to the angle at its corner $a_{0}$ when $\mu$ is even (resp., odd). Then $T_{\mu}$ has the empty $(3,1)$-type when $\mu=0$, the maximal $(3,1)$-type $\{(2,1)\}$ when $\mu>0$, and the multiplicity $\mu_{2,1}=\mu$. 


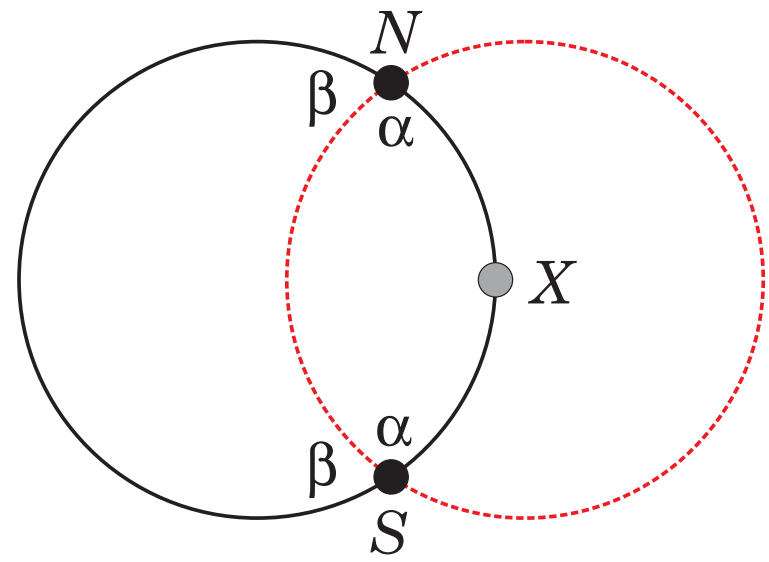

Figure 3. Location of the point $X$.
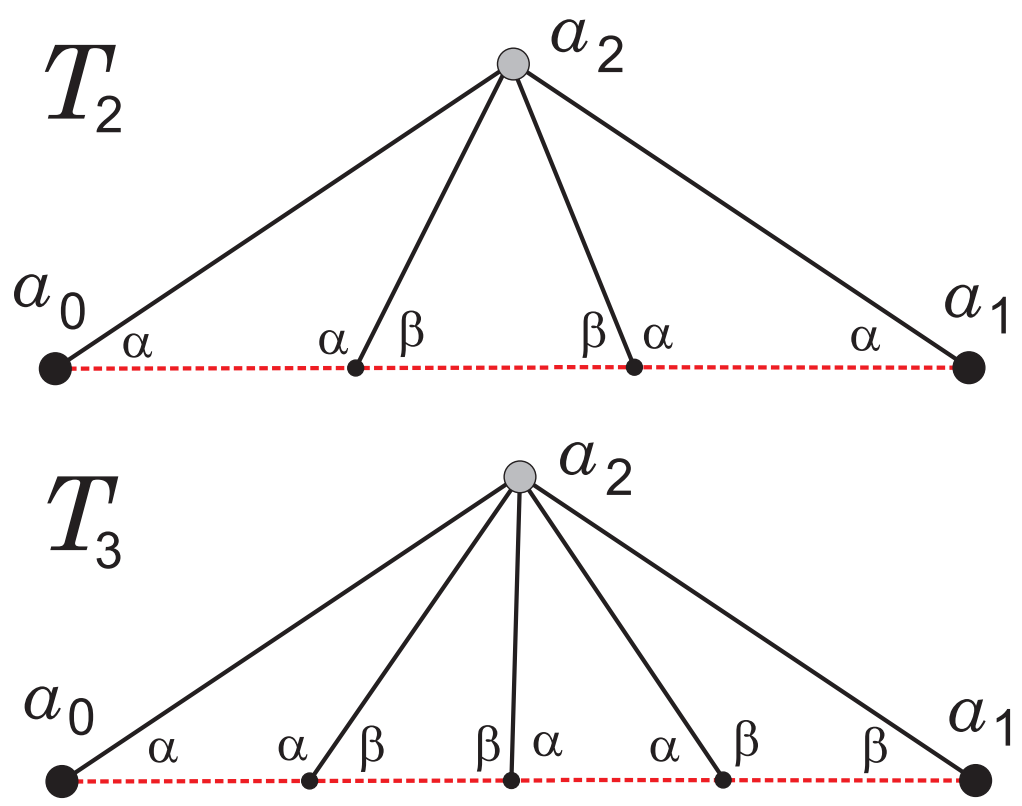

Figure 4. Primitive triangles $T_{\mu}$.

Corollary 8.11. Every primitive triangle with two non-integer corners over the partition $\mathcal{P}$ is combinatorially equivalent to one of the triangles $T_{\mu}$. A triangle $\bar{T}_{\mu}$ with the $(3,2)$-type can be obtained from the triangle $T_{\mu}$ by reflection symmetry, relabeling the corners $a_{1}$ and $a_{2}$.

Let $X$ and $Y$ (see Figure 5) be two points on the same arc of a circle of $\mathcal{P}$ shown by a solid line, inside a disk $\mathbf{D}$ bounded by the circle $C$ of $\mathcal{P}$ shown by the dashed line. For $\mu, \nu \geq 0$, let $R_{\mu \nu}$ (see Figure 6) be a primitive quadrilateral having a non-integer corner $a_{0}$ mapping to $N$, a non-integer corner $a_{1}$ mapped to $S$ (resp., to $N$ ) when $\mu+\nu$ is even (resp., odd), and integer corners $a_{2}$ of order $\nu+1$ and $a_{3}$ 


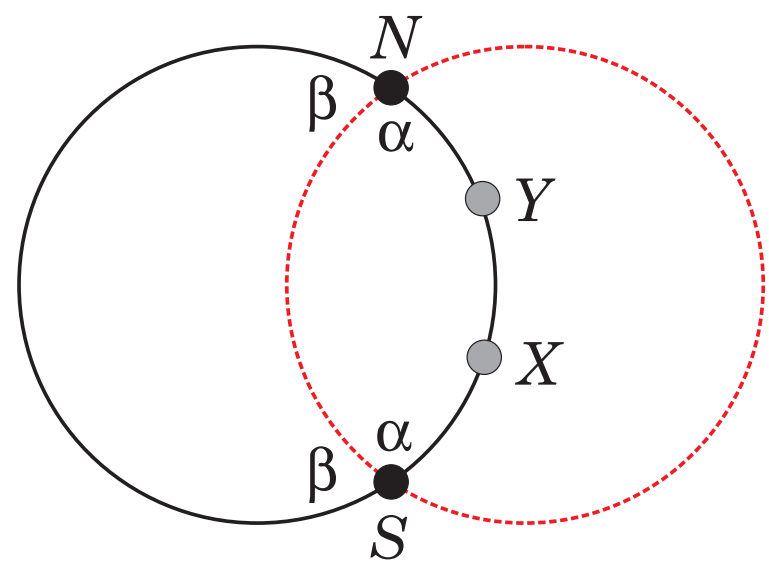

Figure 5. Location of the points $X$ and $Y$ for adjacent integer corners.
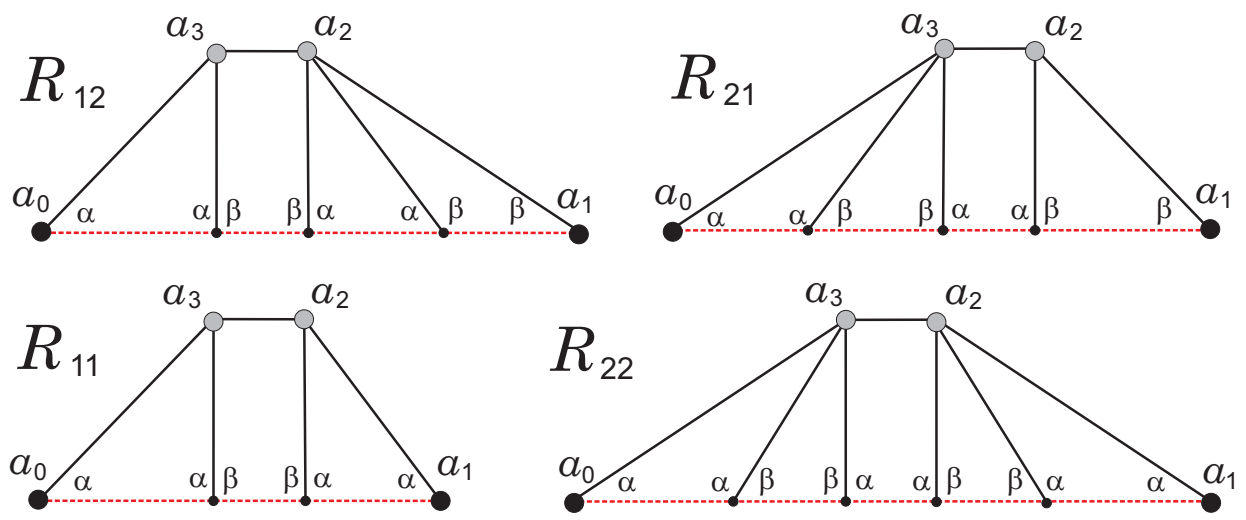

Figure 6. Primitive quadrilaterals $R_{\mu \nu}$.

of order $\mu+1$ mapped to $X$ and $Y$ (resp., to $Y$ and $X$ ) when $\mu$ is even (resp., odd). The side $L_{1}$ of $R_{\mu \nu}$ is mapped to the circle $C$ traversing it counterclockwise. The angle at the corner $a_{1}$ of $R_{\mu \nu}$ is equal (resp., complementary) to the angle at its corner $a_{0}$ when $\mu+\nu$ is even (resp., odd). Then $R_{\mu \nu}$ has the empty $(4,1)$-type when $\mu=\nu=0$, the $(4,1)$-type $\{(3,1)\}$ with the multiplicity $\mu_{3,1}=\mu$ when $\mu>0, \nu=0$, the (4,1)-type $\{(2,1)\}$ with the multiplicity $\mu_{2,1}=\nu$ when $\mu=0, \nu>0$, and the (unique) maximal $(4,1)$-type $\{(3,1),(2,1)\}$ with the multiplicities $\mu_{3,1}=\mu$ and $\mu_{2,1}=\nu$ when $\mu, \nu>0$. Note that when $\mu=0$ (resp., $\nu=0$ ) the corner $a_{3}$ (resp., $a_{2}$ ) of $R_{\mu \nu}$ is removable, thus $R_{\mu \nu}$ is isometric to a triangle (to a digon when $\mu=\nu=0)$.

Corollary 8.12. Every primitive quadrilateral over $\mathcal{P}$ with two adjacent noninteger corners is combinatorially equivalent to one of the quadrilaterals $R_{\mu \nu}$. A quadrilateral $\bar{R}_{\mu \nu}$ with the (4,3)-type can be obtained from the quadrilateral $R_{\mu \nu}$ by reflection symmetry, relabeling the corners $a_{1}, a_{2}, a_{3}$.

Let $X$ and $Y$ (see Figure 7) be two points on distinct circles of $\mathcal{P}$. 
For $\mu, \nu \geq 0$, let $U_{\mu \nu}$ and $\bar{U}_{\mu \nu}$ (see Figure 8 ) be primitive quadrilaterals having a non-integer corner $a_{0}$ mapping to $N$, a non-integer corner $a_{2}$ mapped to $S$ (resp., to $N$ ) when $\mu+\nu$ is even (resp., odd), and integer corners $a_{1}$ of order $\mu+1$ and $a_{3}$ of order $\nu+1$ mapped to $X$ and $Y$, respectively, as shown in Figure $7 \mathrm{f}$ if $\mu$ is even and Figure $7 \mathrm{~b}$ if $\mu$ is odd. The angle at the corner $a_{2}$ of $U_{\mu \nu}$ and $\bar{U}_{\mu \nu}$ is equal (resp., complementary) to the angle at its corner $a_{0}$ when $\mu+\nu$ is even (resp., odd). Then $U_{\mu \nu}$ (resp., $\left.\bar{U}_{\mu \nu}\right)$ has the empty $(4,2)$-type when $\mu=\nu=0$, the $(4,2)$-type $\{(3,1)\}$ (resp., $\{(1,4)\}$ ) with the multiplicity $\mu_{3,1}=\mu$ (resp., $\mu_{1,4}=\mu$ ) when $\mu>0, \nu=$ 0 , the (4,2)-type $\{(1,3)\}$ (resp., $\{(3,2)\}$ ) with the multiplicity $\mu_{1,3}=\nu$ (resp., $\mu_{3,2}=\nu$ ) when $\mu=0, \nu>0$, and the maximal (4,2)-type $\{(3,1),(1,3)\}$ (resp., $\{(1,4),(3,2)\})$ with the multiplicities $\mu_{3,1}=\mu$ and $\mu_{1,3}=\nu$ (resp., $\mu_{1,4}=\mu$ and $\left.\mu_{3,2}=\nu\right)$ when $\mu, \nu>0$. Note that when either $\mu=0$ or $\nu=0$, the quadrilateral $U_{\mu \nu}$ and $\bar{U}_{\mu \nu}$ has a removable integer corner (both integer corners when $\mu=\nu=0$ ) and is isometric to a triangle (a digon when $\mu=\nu=0$ ).

For $\mu, \nu \geq 0$, let $X_{\mu \nu}$ and $\bar{X}_{\mu \nu}$ (see Figure 9) be primitive quadrilaterals having a non-integer corner $a_{0}$ mapping to $N$, a non-integer corner $a_{2}$ mapped to $S$ (resp., to $N$ ) when $\mu+\nu$ is even (resp., odd), and integer corners $a_{1}$ of order 1 and $a_{3}$ of order $\mu+\nu+1$ mapped to $X$ and $Y$, respectively, as shown in Figure 70 if $\mu$ is even and Figure $7 \mathrm{~b}$ if $\mu$ is odd. The angle at the corner $a_{2}$ of $X_{\mu \nu}$ and $\bar{X}_{\mu \nu}$ is equal (resp., complementary) to the angle at its corner $a_{0}$ when $\mu+\nu$ is even (resp., odd). Then $X_{\mu \nu}$ (resp., $\left.\bar{X}_{\mu \nu}\right)$ has the empty $(4,2)$-type when $\mu=\nu=0$, the $(4,2)$ type $\{(3,1)\}$ (resp., $\{(1,4)\}$ ) with the multiplicity $\mu_{3,1}=\mu$ (resp., $\mu_{1,4}=\mu$ ) when $\mu>0, \nu=0$, the $(4,2)$-type $\{(3,2)\}$ (resp., $\{(1,3)\})$ with the multiplicity $\mu_{3,2}=\nu$ (resp., $\mu_{1,3}=\nu$ ) when $\mu=0, \nu>0$, and the maximal $(4,2)$-type $\{(3,1),(3,2)\}$ (resp., $\{(1,4),(1,3)\}$ ) with the multiplicities $\mu_{3,1}=\mu$ and $\mu_{3,2}=\nu$ (resp., $\mu_{1,4}=\mu$ and $\mu_{1,3}=\nu$ ) when $\mu, \nu>0$. Note that the corner $a_{1}$ of $X_{\mu \nu}$ and the corner $a_{3}$ of $\bar{X}_{\mu \nu}$ are removable, thus these quadrilaterals are isometric to triangles $T_{\mu+\nu}$.
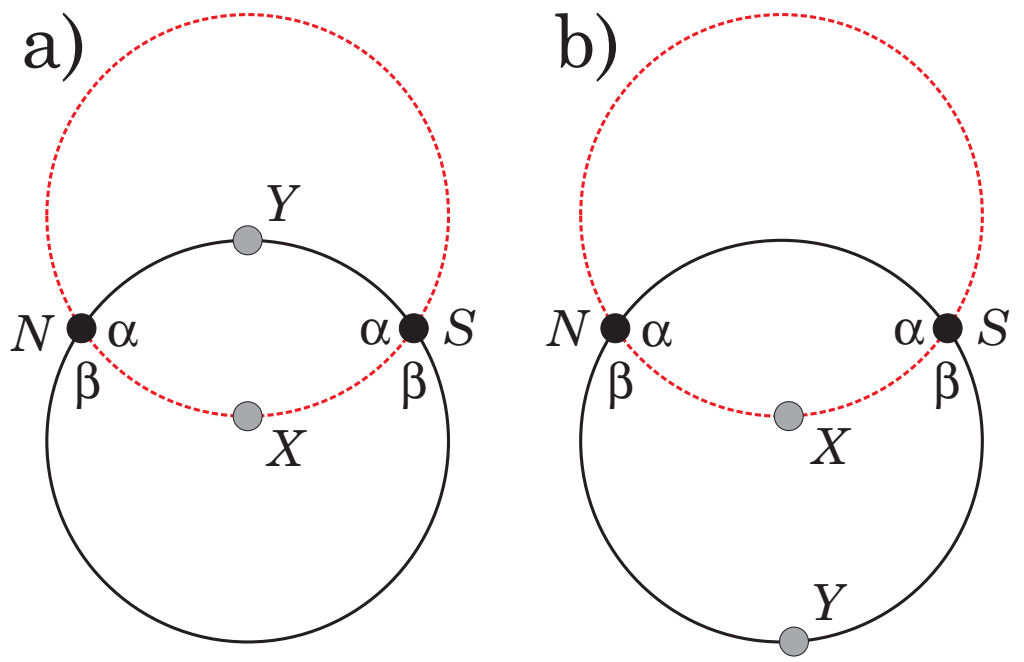

FiguRE 7. Location of the points $X$ and $Y$ for two opposite integer corners. 

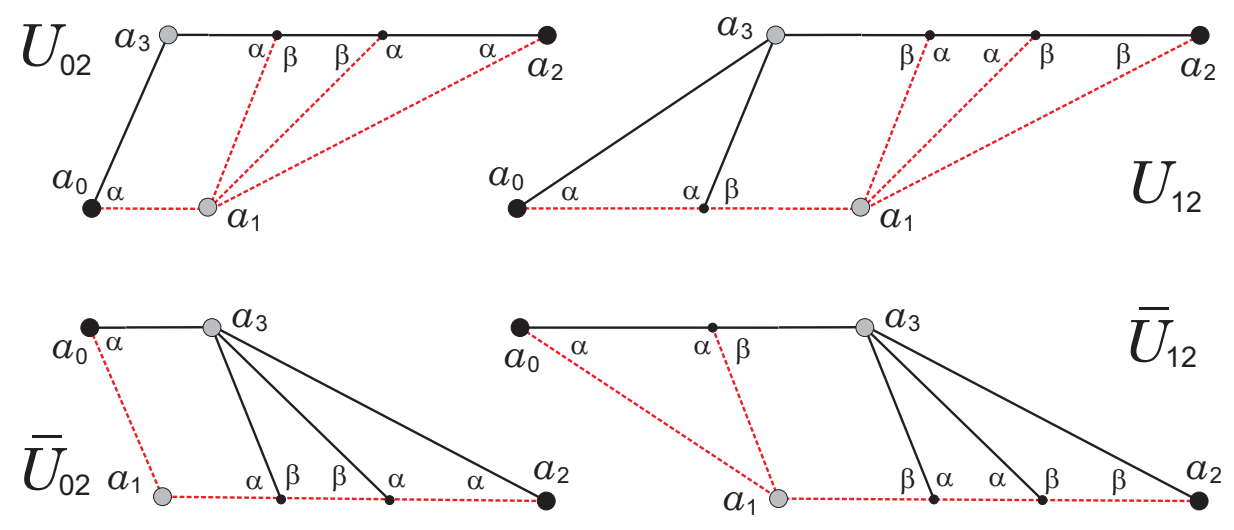

Figure 8. Primitive quadrilaterals $U_{\mu \nu}$ and $\bar{U}_{\mu \nu}$.

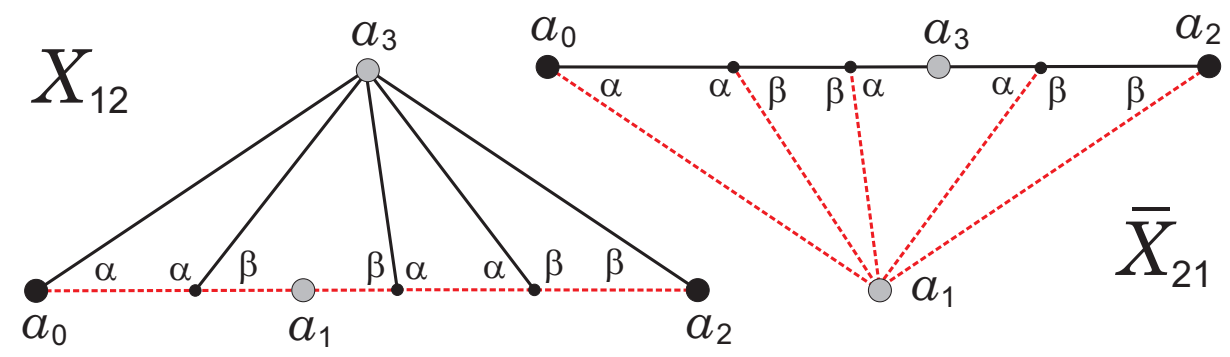

Figure 9. Primitive quadrilaterals $X_{\mu \nu}$ and $\bar{X}_{\mu \nu}$.

Corollary 8.13. Every primitive quadrilateral over $\mathcal{P}$ with two opposite noninteger corners is combinatorially equivalent to one of the quadrilaterals $U_{\mu \nu}, \bar{U}_{\mu \nu}$, $X_{\mu \nu}, \bar{X}_{\mu \nu}$.

Note that $U_{\mu 0}$ and $X_{\mu 0}$ are combinatorially equivalent for each $\mu, U_{0 \nu}$ and $\bar{X}_{0 \nu}$ are combinatorially equivalent for each $\nu, \bar{U}_{\mu 0}$ and $\bar{X}_{\mu 0}$ are combinatorially equivalent for all $\mu$, and $\bar{U}_{0 \nu}$ and $X_{0, \nu}$ are combinatorially equivalent for all $\nu$.

\section{IRREDUCIBLE SPHERICAL POLYGONS WITH TWO NON-INTEGER CORNERS}

In this section, $Q$ is an irreducible marked $n$-gon with two non-integer corners. Its corners and sides are labeled as in the previous section. In particular, the noninteger corners of $Q$ are $a_{0}$ and $a_{k}$. Let $\mathcal{Q}$ be the net of $Q$, and let $\mathcal{Q}_{C}$ and $\mathcal{Q}_{C^{\prime}}$ be preimages of the circles $C$ and $C^{\prime}$ of $\mathcal{P}$, respectively. Since $Q$ is irreducible, its net $\mathcal{Q}$ does not contain an interior arc with the ends at two distinct corners of $Q$. However, $\mathcal{Q}$ may have loops.

Lemma 9.1. Integer corners of $Q$ do not belong to preimages of the vertices of $\mathcal{P}$.

Proof. We proceed by induction on the number $m$ of faces of $\mathcal{Q}$, the case $m=1$ being trivial. Let $p$ be an integer corner of $Q$ that belongs to the preimage of a vertex of $\mathcal{P}$. Then there is a maximal interior $\operatorname{arc} \gamma$ of $\mathcal{Q}$ with an end at $p$, such that 
$\gamma$ partitions the angle at $p$ into two non-integer angles. The other end $q$ of $\gamma$ cannot be on a side of $Q$. Otherwise, $\gamma$ would partition $Q$ into two irreducible polygons, each having non-integer angles at both $p$ and $q$, which contradicts Theorem 7.1, Thus $q=p$ and $\gamma$ is a loop. Let $C$ be the circle of $\mathcal{P}$ to which $\gamma$ maps, and let $C^{\prime}$ be the other circle of $\mathcal{P}$. Then there is a maximal interior arc $\gamma^{\prime}$ of $\mathcal{Q}_{C^{\prime}}$ having one end at $p$ and intersecting $\gamma$ in an interior vertex $q$ of $\mathcal{Q}$. We saw in the proof of Theorem 7.1 that $\gamma^{\prime}$ cannot be a loop. Thus $\gamma^{\prime}$ partitions $Q$ into two irreducible polygons, each of them having less than $m$ faces in its net, with $p$ being an integer corner of each of them. This contradicts the induction hypothesis.

Lemma 9.2. Let $\gamma$ be a loop of $\mathcal{Q}$. Then $\gamma$ contains an integer corner of $Q$.

Proof. Let $\gamma$ be a loop in $\mathcal{Q}_{C}$ that does not contain a corner of $Q$. Then $\gamma$ intersects $\mathcal{Q}_{C^{\prime}}$ at two points $q$ and $q^{\prime}$. Let $\gamma^{\prime}$ be the maximal loop in $\mathcal{Q}_{C^{\prime}}$ passing through $q$ and $q^{\prime}$. The same arguments as in the proof of Theorem 7.1 show that $\gamma^{\prime}$ cannot be a loop. Since $Q$ is irreducible, $\gamma^{\prime}$ cannot have both ends at the corners of $Q$. Thus one of its ends is at the side of $Q$. But this implies that a face of $\mathcal{Q}$ adjacent to $\gamma$ outside the disk bounded by $\gamma$ has a disconnected intersection with $\mathcal{Q}_{C}$, a contradiction.

Lemma 9.3. Let $\gamma$ be a loop of $\mathcal{Q}_{C}$ containing an integer corner $p$ of $Q$. Then there is a loop $\gamma^{\prime}$ of $\mathcal{Q}_{C^{\prime}}$ intersecting $\gamma$ at two points and containing an integer corner $p^{\prime}$ of $Q$. The union of $\gamma$ and $\gamma^{\prime}$ is a pseudo-diagonal of $Q$ shown in Figure 2,

Proof. Let $\gamma^{\prime}$ be a maximal arc of $\mathcal{Q}_{C^{\prime}}$ intersecting $\gamma$ at two points. Then $\gamma^{\prime}$ cannot have both ends on the sides of $Q$, since that would contradict Theorem 7.1. Also, $\gamma^{\prime}$ cannot have one end at a corner and another one on a side of $Q$, since then $\mathcal{Q}$ would have a face with disconnected intersection of its boundary with $\mathcal{Q}_{C}$. Since $Q$ is irreducible, $\gamma^{\prime}$ must be a loop. Lemma 9.2 implies that $\gamma^{\prime}$ contains an integer corner $p^{\prime}$ of $Q$.

Definition 9.4. The number $\nu_{i j}$ of the pseudo-diagonals of $Q$ (see Figure 2) connecting integer corners $a_{i}$ and $a_{j}$ of $Q$ is called the multiplicity of a pseudo-diagonal connecting $a_{i}$ and $a_{j}$.

Theorem 9.5. Each irreducible spherical polygon $Q$ with two non-integer corners can be obtained from a primitive polygon by adding (multiple) pseudo-diagonals connecting some of its integer corners. These pseudo-diagonals do not intersect inside $Q$. For a primitive polygon with a maximal type, the irreducible polygons that can be obtained from it are uniquely determined by the multiplicities of the pseudo-diagonals.

Proof. The first part of the statement is obvious, since removing all loops from the net of $Q$ we obtain a primitive polygon $Q^{\prime}$. If $Q^{\prime}$ has maximal type, then each face of its net $\mathcal{Q}^{\prime}$ has at most two integer corners of $Q^{\prime}$ in its boundary. The multiplicities of the pseudo-diagonals connecting these pairs of corners completely determine the irreducible polygon $Q$ from which $Q^{\prime}$ was obtained.

Each of the primitive quadrilaterals $U_{\mu \nu}, \bar{U}_{\mu \nu}, X_{\mu \nu}, \bar{X}_{\mu \nu}$ (see Figures 89) contains a single face $F$ of its net with both integer corners $a_{1}$ and $a_{3}$ in its boundary. Adding $\kappa$ pseudo-diagonals connecting the two integer corners inside $F$, we obtain irreducible quadrilaterals $U_{\mu \nu}^{\kappa}, \bar{U}_{\mu \nu}^{\kappa}, X_{\mu \nu}^{\kappa}, \bar{X}_{\mu \nu}^{\kappa}$. For $\kappa>0$, these quadrilaterals are not primitive. We identify $U_{\mu \nu}^{0}, \bar{U}_{\mu \nu}^{0}, X_{\mu \nu}^{0}, \bar{X}_{\mu \nu}^{0}$ with $U_{\mu \nu}, \bar{U}_{\mu \nu}, X_{\mu \nu}, \bar{X}_{\mu \nu}$, respectively. 
Corollary 9.6. Every marked irreducible quadrilateral over $\mathcal{P}$ with two opposite non-integer corners is combinatorially equivalent to one of the quadrilaterals $U_{\mu \nu}^{\kappa}$, $\bar{U}_{\mu \nu}^{\kappa}, X_{\mu \nu}^{\kappa}, \bar{X}_{\mu \nu}^{\kappa}$ with $\mu, \nu, \kappa \geq 0$.

Note that $U_{\mu 0}^{\kappa}$ and $X_{\mu 0}^{\kappa}$ are combinatorially equivalent for each $\mu, \kappa, U_{0 \nu}^{\kappa}$ and $\bar{X}_{0 \nu}^{\kappa}$ are combinatorially equivalent for each $\nu, \kappa, \bar{U}_{\mu 0}^{\kappa}$ and $\bar{X}_{\mu 0}^{\kappa}$ are combinatorially equivalent for all $\mu, \kappa, \bar{U}_{0 \nu}^{\kappa}$ and $X_{0, \nu}^{\kappa}$ are combinatorially equivalent for all $\nu, \kappa$.

Definition 9.7. The $(n, k)$-type of an irreducible polygon $Q$ (or simply the type of $Q$ if $n$ and $k$ are not specified) is the type of the primitive polygon $Q^{\prime}$ obtained from $Q$ by removing all loops, with the unordered pairs $(i, j)$ added for the integer corners $a_{i}$ and $a_{j}$ with positive multiplicities $\nu_{i j}$ of the pseudo-diagonals connecting these corners. The type of $Q$ is maximal if the type of $Q^{\prime}$ is maximal, and any two integer corners of $Q^{\prime}$ belonging to the boundary of the same face of its net are connected in $Q$ by at least one pseudo-diagonal. Every $(n, k)$-type of an irreducible spherical polygon can be obtained from a (non-unique) maximal $(n, k)$-type if some of the multiplicities are allowed to be zero.

Proposition 9.8. The number of distinct maximal $(n, k)$-types of irreducible spherical polygons equals the number of distinct maximal $(n, k)$-types of primitive spherical polygons.

Proof. This follows from Theorem 9.5.

\section{Classification of SPHERICAL Digons AND TRIANGleS}

For $m \geq 1$, there is a unique, up to combinatorial equivalence, rational spherical digon $D_{m}$, with the angles at each of its corners equal to $m \pi$. The two sides $L_{1}$ and $L_{2}$ of $D_{m}$ have the same lengths $l_{1}=l_{2}<2 \pi$ when $m$ is even, and complementary lengths $l_{1}+l_{2}=2 \pi$ when $m$ is odd.

There is a unique, up to combinatorial equivalence, irreducible spherical digon with two equal non-integer corners, isometric to a face of $\mathcal{P}$. Any irrational spherical digon is obtained from it by attaching a digon $D_{m}$. Here $m \geq 0$, with $m=0$ meaning that nothing is attached.

Before proceeding with classification of spherical triangles, we describe a simple operation on an arbitrary spherical polygon $Q$ : attaching a digon to a "short" side of $Q$. Here a side $L$ of $Q$ is called short if it is mapped to a proper arc of a circle of $\mathcal{P}$. Alternatively, $L$ is short if the length of $L$ in less than $2 \pi$. Otherwise, $L$ is called long. If $L$ is a short side of $Q$, we can construct a polygon $Q^{\prime}=Q \cup_{L} D_{m}$ with the same corners as $Q$ by attaching to $L$ a rational digon $D_{m}$ with a side of the same length as $L$. Note that the "free" side $L^{\prime}$ of $D_{m}$ is a short side of $Q^{\prime}$. Attaching a digon $D_{n}$ to $L^{\prime}$ is equivalent to attaching a digon $D_{m+n}$ to $L$.

Theorem 9.5 implies that any irreducible spherical triangle with two non-integer corners is primitive and combinatorially equivalent to one of the triangles $T_{\mu}$ (see Corollary 8.11). A rational irreducible triangle is combinatorially equivalent to a rational triangle $\nabla$ with three removable corners.

Any spherical triangle $T$ over $\mathcal{P}$ is either rational or has two non-integer corners. If $T$ has two non-integer corners $a_{0}$ and $a_{1}$, it is combinatorially equivalent to a triangle $T_{\mu}$ with digons $D_{i}, D_{j}$ and $D_{l}$ attached to its sides $L_{1}, L_{2}$ and $L_{3}$, respectively, where $i, j, l \geq 0$, the value 0 meaning that no digon is attached, and $i>0$ only when $\mu=0$. 


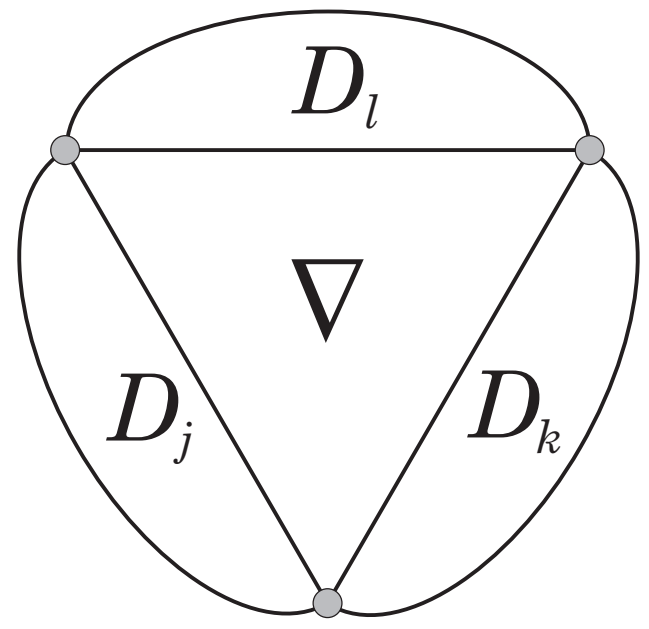

Figure 10. Rational triangle $\nabla_{j k l}$.

If $T$ is rational, it is combinatorially equivalent to a rational triangle $\nabla$ with digons $D_{j}, D_{k}$ and $D_{l}$ attached to its three sides, where $j, k, l \geq 0$ are determined by $T$ uniquely up to cyclic permutation. We use notation $\nabla_{j k l}$ for such a triangle (see Figure 10).

\section{Classification of SPherical QUadrilaterals WITH TWO ADJACENT NON-INTEGER CORNERS}

We consider marked quadrilaterals over $\mathcal{P}$ with two adjacent non-integer corners $a_{0}$ and $a_{1}$.

Lemma 11.1. A quadrilateral $Q$ over $\mathcal{P}$ cannot have more than two non-integer corners.

Proof. If $Q$ is a union of irreducible polygons, then one of them, say $\tilde{Q}$, should be either a quadrilateral or a triangle. If $\tilde{Q}$ is a quadrilateral, then, by Lemma 9.1 . $\tilde{Q}$ must have two corners that are not mapped to vertices of $\mathcal{P}$. These corners of $\tilde{Q}$, and the corresponding corners of $Q$, must be integer. If $\tilde{Q}$ is a triangle, at least one of its corners is not mapped to a vertex of $\mathcal{P}$, thus $Q$ has at least one integer corner. Since the number of non-integer corners of any polygon over $\mathcal{P}$ is even, at least two corners of $Q$ are integer.

A quadrilateral $Q$ can be partitioned into irreducible polygons. We'll see later that for a quadrilateral with two adjacent non-integer corners this partition is unique.

If one of these polygons is a triangle $T_{\mu}$ with its corners at $a_{0}, a_{1}$ and either $a_{2}$ or $a_{3}$, then $Q$ is a union of $T_{\mu}$, a triangle $\nabla_{j k l}$ attached to its side other than $L_{1}$ so that $D_{j}$ is adjacent to $T_{\mu}$, and digons $D_{i}$ and $D_{m}$ attached to the other two sides of $T_{\mu}$ so that $D_{i}$ is adjacent to its side $L_{1}$ (see Figures 11 a-b). Here $i, j, k, l, m \geq 0$, with $i>0$ only if $\mu=0$.

Otherwise, $Q$ contains a quadrilateral $R_{\mu \nu}$ having the same corners as $Q$. Then $Q$ is the union of $R_{\mu \nu}$ and digons $D_{i}, D_{k}, D_{l}, D_{m}$ attached to its sides (see Figure 11k). Here $i, j, k, l \geq 0$, with $i>0$ only if $\mu=\nu=0$. 


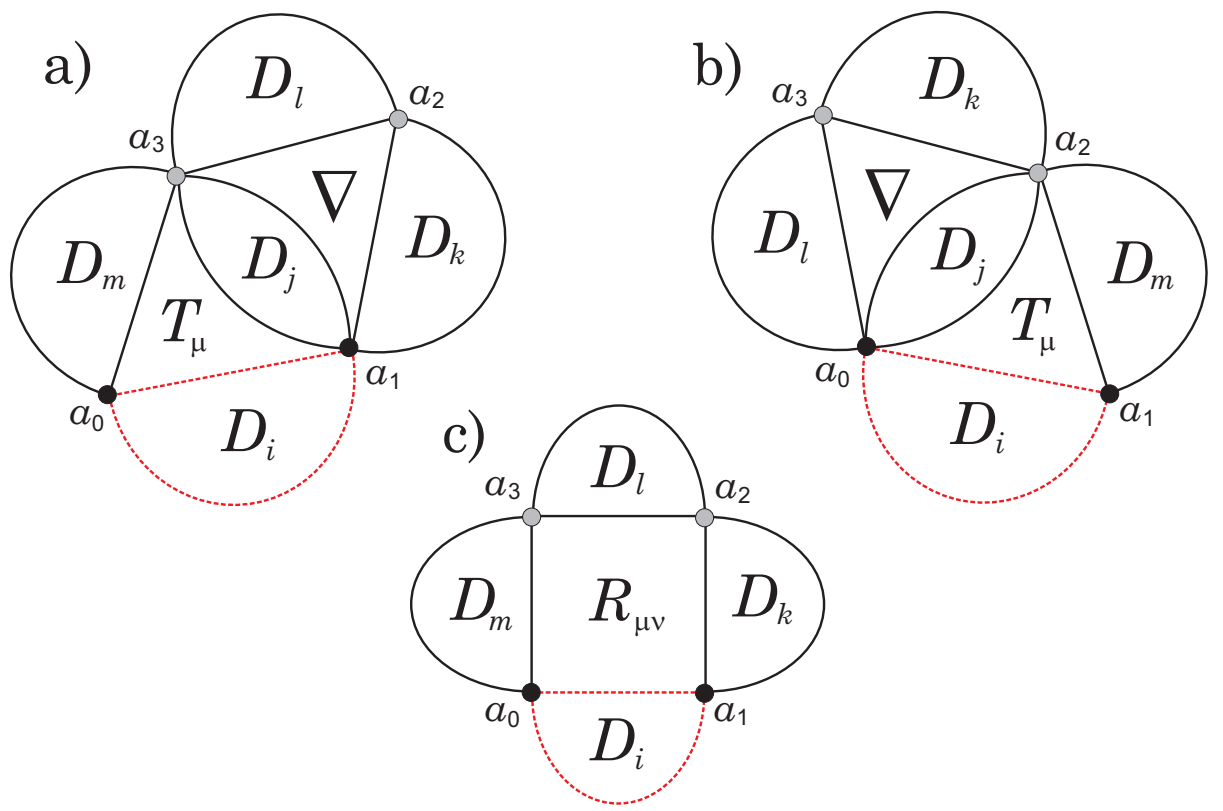

FIGURE 11. Quadrilaterals with adjacent non-integer corners.

a)

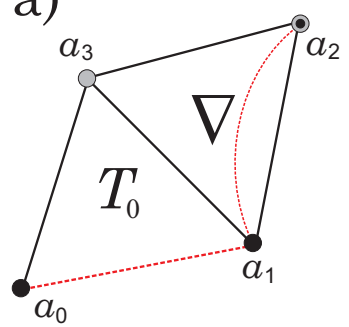

c)

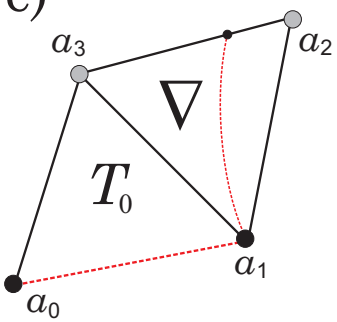

b)

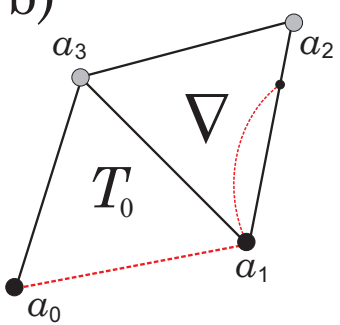

d)

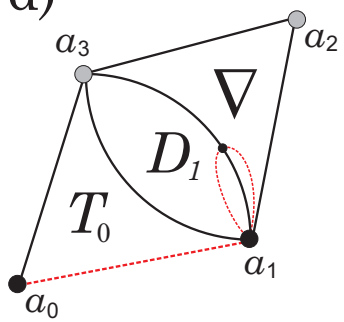

FiguRe 12. Quadrilaterals in Figure 11a with $j=0$ (a-c) and $j=1(\mathrm{~d})$. 
Note that a vertex of $\nabla$ that is not a vertex of $T_{\mu}$ may be mapped to a vertex of $\mathcal{P}$ when $j$ is even, but not when $j$ is odd. Figures 12 a-c shows the complete net for the quadrilateral in Figure 11] with $j=0$ (and $\mu=i=k=l=m=0$ ). All these quadrilaterals are combinatorially equivalent, although the corner $a_{2}$ is mapped to a vertex of $\mathcal{P}$ in Figure $12 \mathrm{a}$ but not in Figures $12 \mathrm{~b}-\mathrm{c}$. Figure $12 \mathrm{~d}$ shows the complete net for the quadrilateral in Figure 11 w with $j=1$. In this case, the corner $a_{2}$ cannot be mapped to a vertex of $\mathcal{P}$, since there are two points mapped to vertices of $\mathcal{P}$ on the side of $\nabla$ connecting $a_{1}$ and $a_{3}$.

Counting quadrilaterals with given angles. We want to classify marked spherical quadrilaterals $Q$ with two adjacent non-integer corners $a_{0}$ and $a_{1}$, and with given orders $A_{0}, \ldots, A_{3}$ of its corners, up to combinatorial equivalence. Here $A_{0}, A_{1} \geq 0$ and $A_{2}, A_{3}>0$ are the orders of non-integer and integer corners of $Q$, respectively.

We define the following numbers:

$$
\begin{aligned}
\delta & =\frac{1}{2}\left(A_{1}+A_{3}-A_{0}-A_{2}\right), \\
\sigma & =\frac{1}{2}\left(A_{2}+A_{3}-A_{0}-A_{1}\right), \\
\rho & =\frac{1}{2}\left(A_{2}+A_{3}-\left|A_{1}-A_{0}\right|\right) .
\end{aligned}
$$

These numbers are integer if and only if the corners $a_{0}$ and $a_{1}$ of $Q$ are mapped to distinct vertices of $\mathcal{P}$.

The relations of these parameters with those in Theorem 5.3 is the following:

$$
A_{0}=\left[\alpha_{3}\right], \quad A_{1}=\left[\alpha_{0}\right], \quad A_{2}=\alpha_{1}, \quad A_{3}=\alpha_{2},
$$

In case a), $\rho=\kappa+1$, and in case b) $\sigma=\kappa+3 / 2$.

Lemma 11.2. For given positive integers $p, q, r, s$ satisfying $p+r=q+s$, the system of equations $x+y=p+1, y+z=q+1, z+t=r+1, t+x=s+1$ has $\min (p, q, r, s)$ solutions $(x, y, z, t)$ in positive integers.

The proof is left as an easy exercise for the reader.

Proposition 11.3. A marked quadrilateral $Q$ with non-integer corners $a_{0}$ and $a_{1}$ mapped to distinct vertices of $\mathcal{P}$ exists if and only if $\rho$ is a positive integer. In this case, there are $\min \left(A_{2}, A_{3}, \rho\right)$ combinatorially distinct quadrilaterals with given orders $A_{0}, \ldots, A_{3}$ of their angles.

$A$ quadrilateral $Q$ with the corners $a_{0}$ and $a_{1}$ mapped to the same vertex of $\mathcal{P}$ exists if and only if $\sigma-1$ is a positive non-integer. In this case, there are $\min \left(A_{2}, A_{3},[\sigma]\right)$ combinatorially distinct quadrilaterals with given angles.

Proof. For a quadrilateral $Q$ shown in Figure 11 , its corners $a_{0}$ and $a_{1}$ are mapped to distinct vertices of $\mathcal{P}$ if $\mu$ is even, and to the same vertex of $\mathcal{P}$ if $\mu$ is odd. Orders of the corners of $Q$ are $A_{0}=i+m, A_{1}=i+j+k+1, A_{2}=k+l+1$, $A_{3}=j+\mu+l+m+1$. We have $\delta=j+1+\mu / 2, \sigma=l-i+1+\mu / 2$. If $A_{1}>A_{0}$, that is, $j+k \geq m$, then $\rho=l+m+1+\mu / 2$, otherwise, $\rho=j+k+l+2+\mu / 2>A_{2}$. In particular, $\delta$ is positive. Similarly, $\delta$ is negative for the quadrilateral $Q$ shown in Figure 11b.

For the quadrilateral $Q$ in Figure 11k, its corners $a_{0}$ and $a_{1}$ are mapped to distinct vertices of $\mathcal{P}$ if $\mu+\nu$ is even, and to the same vertex of $\mathcal{P}$ if $\mu+\nu$ is 
odd. Orders of the corners of $Q$ are $A_{0}=i+m, A_{1}=i+k, A_{2}=k+l+1+\nu$, $A_{3}=l+m+1+\mu$. We have $\delta=(\mu-\nu) / 2, \sigma=l-i+1+(\mu+\nu) / 2$. If $A_{1} \geq A_{0}$, that is, $k \geq m$, then $\rho=l+m+1+(\mu+\nu) / 2$, otherwise $\rho=k+l+1+(\mu+\nu) / 2$.

Note that in all cases $\delta, \sigma$ and $\rho$ are integer if and only if the corners $a_{0}$ and $a_{1}$ are mapped to distinct vertices of $\mathcal{P}$. If $i>0$, then $\mu=0$ in Figures $11 \mathrm{~b}-\mathrm{b}$ and $\mu=\nu=0$ in Figure 11k, thus the fractional parts of the angles at $a_{0}$ and $a_{1}$ are equal. If $i=0$, then $\sigma \geq 1$.

We start with the quadrilaterals with equal fractional parts of the angles at $a_{0}$ and $a_{1}$.

Consider first the case $\delta=0$, that is, $A_{3}-A_{0}=A_{2}-A_{1}$. This is only possible for a quadrilateral $Q$ in Figure 11 c with $\mu=\nu$. We may assume $A_{0}-A_{1}=A_{3}-A_{2} \geq 0$ (the other case follows by symmetry). Then $A_{3} \geq A_{2}$ and $\rho=\left(A_{3}+A_{2}-A_{0}+\right.$ $\left.A_{1}\right) / 2=A_{2}$, so we have to prove that the number of combinatorially distinct quadrilaterals is $A_{2}$.

Lemma 11.2 applied to $x=i, y=m+1, z=l+1, t=k+1$ implies that the number of quadrilaterals with $i>0$ is $\min \left(A_{0}, A_{1}, A_{2}, A_{3}\right)=\min \left(A_{1}, A_{2}\right)$. If $A_{1} \geq A_{2}$, the number of quadrilaterals is $A_{2}$. Otherwise, there are $A_{2}-A_{1}=$ $A_{3}-A_{0}$ quadrilaterals with $i=0, m=A_{0}, k=A_{1}, 0 \leq \mu=\nu<A_{2}-A_{1}$, $l=A_{2}-A_{1}-1-\nu=A_{3}-A_{0}-1-\mu$. Thus the total number of quadrilaterals is again $A_{2}$.

Consider now the case $\delta>0$ (the case $\delta<0$ follows by symmetry). Then $A_{3}>A_{2}+A_{0}-A_{1}$, so $A_{3}>A_{2}$ and $\rho=\left(A_{2}+A_{3}+A_{1}-A_{0}\right) / 2=A_{2}+\delta>A_{2}$ if $A_{0} \geq A_{1}$. If $A_{0}<A_{1}$, then $\rho=\left(A_{2}+A_{3}+A_{0}-A_{1}\right) / 2=A_{3}-\delta<A_{3}$. Thus we have to prove that the number of quadrilaterals is $\min \left(A_{2}, A_{3}-\delta\right)$.

The following three subcases are possible:

(i) a quadrilateral in Figure 11; with $i=0$ and $\mu>\nu$,

(ii) a quadrilateral in Figure 11] with $i=0$,

(iii) a quadrilateral in Figure 11 with $i>0$ and $\nu=0$.

Subcase (i). For a quadrilateral $Q$ in Figure 11, with $i=0$ and $\mu>\nu$, we have $m=A_{0}, k=A_{1}, A_{2}>A_{1}$. For each $\nu$ such that $0 \leq \nu<A_{2}-A_{1}$, there is a quadrilateral with $l=A_{2}-A_{1}-1-\nu$ and $\mu=\nu+2 \delta$. Thus there are $A_{2}-A_{1}$ quadrilaterals in this case.

Subcase (ii). For a quadrilateral $Q$ in Figure 11] with $i=0$, we have $m=A_{0}$, $j+k+1=A_{1}, k+l+1=A_{2}, \mu+j+l+2=A_{3}-A_{0}, \delta=j+1+\mu / 2$, $\sigma=l+1+\mu / 2>0$. For a fixed even $\mu \geq 0$, we have $j=\delta-1-\mu / 2, l=\sigma-1-\mu / 2$, $k=A_{1}-\delta+\mu / 2$. Thus $\delta-A_{1} \leq \mu / 2 \leq \min (\delta, \sigma)$. Since $\mu$ is even, the number of quadrilaterals in this case is $\min \left(A_{1}, A_{2}\right)$ if $A_{1} \leq \delta$ and $\min (\delta, \sigma)$ if $A_{1}>\delta$. Note that $\sigma-\delta=A_{2}-A_{1}$.

Subcase (iii). For a quadrilateral $Q$ in Figure 11] with $i>0$ and $\mu=0$, we have $\delta=j+1, i+m=A_{0}, i+k=A_{1}-\delta, k+l+1=A_{2}, l+m+1=A_{3}-\delta$. Lemma 11.2 applied to $x=i, y=m+1, z=l+1, t=k+1$ implies that the number of quadrilaterals is $\min \left(A_{0}, A_{1}-\delta, A_{2}, A_{3}-\delta\right)$. If $A_{2}>A_{1}$, that is, $l>i+j$, then $A_{3}-\delta>A_{0}$ and the number of quadrilaterals is $\min \left(A_{0}, A_{1}-\delta\right)$.

Combining subcases (i)-(iii), we see first that, if $A_{2}>A_{1}$ and $A_{1} \leq \delta$, then the number of quadrilaterals is $A_{2}$ (there are no quadrilaterals in the subcase (iii) in this case). Note that $A_{3}-\delta=\left(A_{3}+A_{2}+A_{0}-A_{1}\right) / 2=A_{2}+\delta-A_{1} \geq A_{2}$ in this case. 
Next, if $A_{2}>A_{1}$ and $A_{1}>\delta$, the total number of quadrilaterals is $\min \left(A_{2}-A_{1}+\delta+A_{0}, A_{2}\right)=\min \left(A_{3}-\delta, A_{2}\right)$.

Finally, if $A_{2} \leq A_{1}$, then there are no quadrilaterals in the subcase (i). If $A_{1} \leq \delta$, there are no quadrilaterals in the subcase (iii), and the number of quadrilaterals in the subcase (ii) is $A_{2}$. If $A_{1}>\delta$ and $\sigma>0$, then the number of quadrilaterals in the subcase (ii) is $\sigma$. Since $A_{0}+\sigma=A_{3}-\delta$ and $A_{1}-\delta+\sigma=A_{2}$ the total number of quadrilaterals is $\min \left(A_{2}, A_{3}-\delta\right)$. If $\sigma \leq 0$, there are no quadrilaterals in subcases (i) and (ii), $A_{0} \geq A_{2}+A_{3}-A_{1} \geq A_{3}$ and $A_{1}-\delta \geq A_{2}$. Thus the number of quadrilaterals in (iii) is $\min \left(A_{2}, A_{3}-\delta\right.$ ).

If the fractional parts of the angles at $a_{0}$ and $a_{1}$ are complementary, then $i=0$. Since $\delta \neq 0$ in this case, we may assume $\delta>0$ (the other case follows by symmetry). Since $i=0$, only subcases (i) and (ii) above are possible.

Repeating the above arguments, we see that $A_{2}>A_{1}$ in the subcase (i), and the number of quadrilaterals is $A_{2}-A_{1}$. In the subcase (ii), since $\mu \geq 1$ is odd, the number of quadrilaterals is $\min \left(A_{1}, A_{2}\right)$ when $A_{1}<\delta$ and $\min ([\delta],[\sigma])$ when $A_{1}>\delta$.

Thus the total number of quadrilaterals is $A_{2}$ if $A_{1}<\delta$. Since $A_{2}-A_{1}=\sigma-\delta$, we have $\sigma>A_{2}$ in this case.

When $A_{1}>\delta$, either $A_{2} \leq A_{1}$ and the total number of quadrilaterals is [ $\sigma$ ] (since there are no quadrilaterals in the subcase (i)) or $A_{2}>A_{1}$ and the total number of quadrilaterals is again $[\sigma]=A_{2}-A_{1}+\delta$. Note that $\sigma<A_{2}$ when $A_{1}>\delta$.

This completes the proof.

\section{Classification of SPherical Quadrilaterals WITH TWO OPPOSITE NON-INTEGER CORNERS}

We consider now marked quadrilaterals $Q$ with two opposite non-integer corners $a_{0}$ and $a_{2}$. We assume that the corner $a_{0}$ is mapped to the vertex $N$ of $\mathcal{P}$. The corner $a_{2}$ may be mapped either to the vertex $S$ or to the vertex $N$, depending on the net of $Q$ (see Corollary 8.13). For an irreducible quadrilateral $Q$, the integer corners $a_{1}$ and $a_{3}$ are mapped to the points $X$ and $Y$ on two distinct circles of $\mathcal{P}$ as shown on Figure 7. However, for a reducible quadrilateral $Q$ one of these corners may be mapped to a vertex of $\mathcal{P}$. Note that a partition of such a quadrilateral $Q$ into irreducible polygons may be non-unique.

Example 12.1. Consider the quadrilaterals $I, J, K$ shown in Figures 13-b-c. The quadrilateral $I$ can be represented in two different ways as the union of a primitive quadrilateral (either $X_{01}$ or $\bar{X}_{01}$ ) and a digon $D_{1}$ attached to its side of order 2 . The quadrilateral $J$ can be represented in two different ways as the union of a primitive quadrilateral (either $X_{10}$ or $\bar{X}_{10}$ ) and a digon $D_{1}$ attached to its side of order 2. The quadrilateral $K$ can be represented in two different ways as the union of a primitive quadrilateral (either $X_{22}$ or $\bar{X}_{22}$ ) and two digons $D_{1}$ attached to its adjacent sides of order 2 . Alternatively, $K$ can be represented in two different ways as the union of a primitive quadrilateral (either $U_{22}$ or $\bar{U}_{22}$ ) and two digons $D_{1}$ attached to its opposite sides of order 2 .

A reducible quadrilateral $Q$ may contain an irreducible quadrilateral with the same corners as $Q$. If an arc of the net $\mathcal{Q}$ of such a quadrilateral connects its two corners, these corners must be adjacent. Alternatively, if $Q$ does not contain an irreducible quadrilateral, then there is an arc of $\mathcal{Q}$ connecting two opposite 
a)

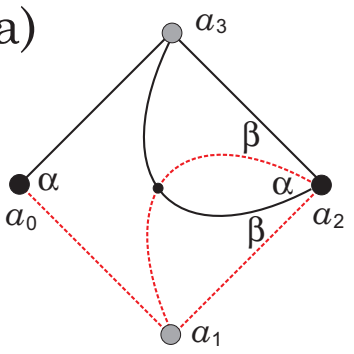

b)

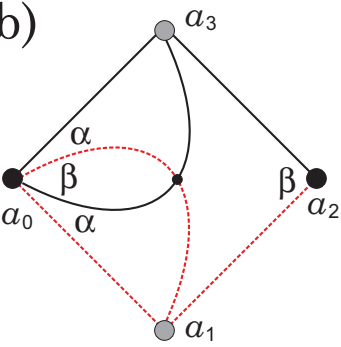

c)

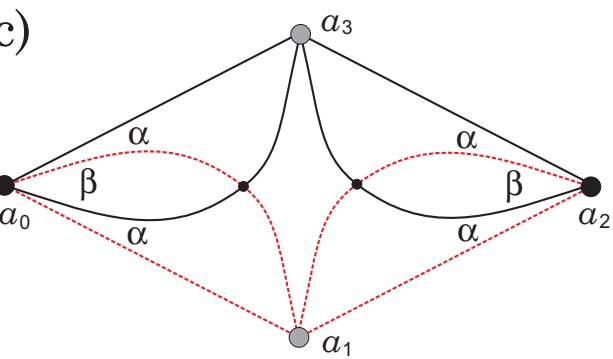

FigURE 13. Non-unique partition into irreducible polygons.

a)

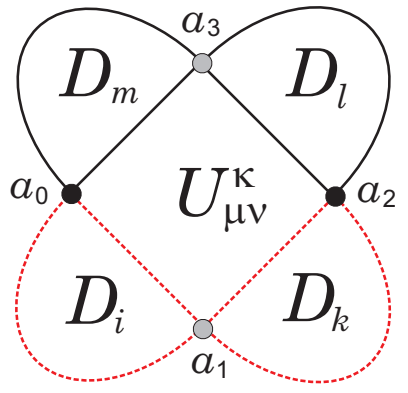

b)

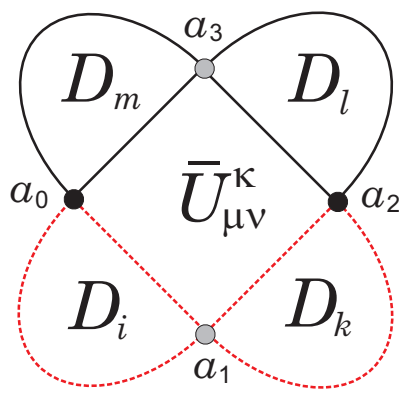

Figure 14. Quadrilaterals of the types $U$ and $\bar{U}$.

a)

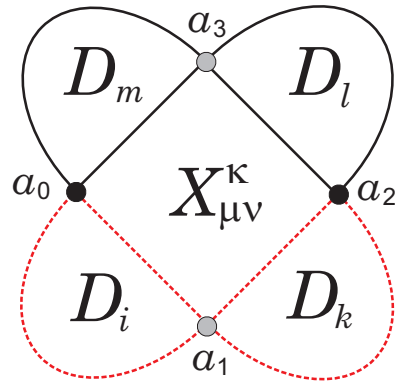

b)

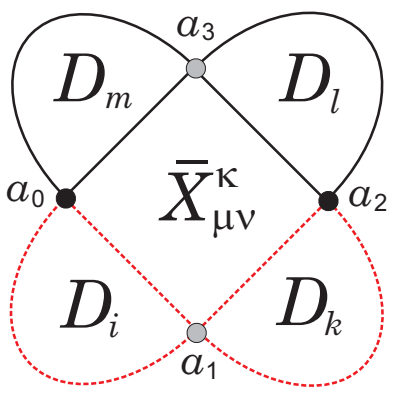

Figure 15. Quadrilaterals of the types $X$ and $\bar{X}$. 
a)

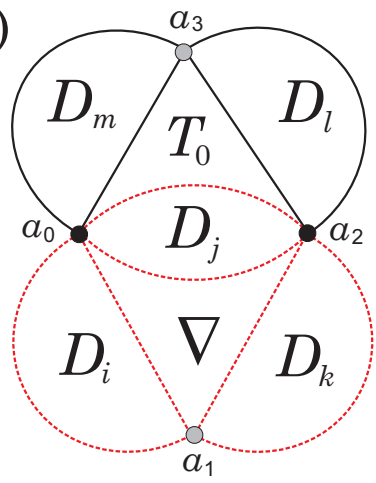

b)
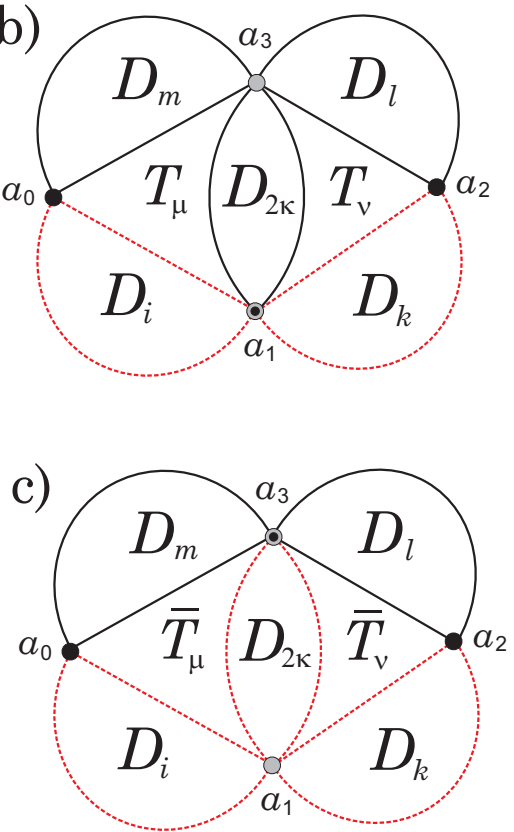

Figure 16. Quadrilaterals of the types $T \nabla, T T$ and $\overline{T T}$.

corners of $Q$, thus $Q$ is a union of two triangles glued together along their common side. Classification of irreducible quadrilaterals in Corollary 9.6. and classification of spherical triangles in section [10, imply the following classification of spherical quadrilaterals with two opposite non-integer corners:

Proposition 12.2. Every marked spherical quadrilateral $Q$ over partition $\mathcal{P}$ with two opposite non-integer corners, one of them marked as $a_{0}$, containing an irreducible quadrilateral, is combinatorially equivalent to one of the following:

Type $U$. An irreducible quadrilateral $U_{\mu \nu}^{\kappa}$ with digons $D_{i}, D_{k}, D_{l}, D_{m}$ attached to its sides $L_{1}, L_{2}, L_{3}, L_{4}$ (see Figure 14a). Here $i, k, l, m, \mu, \nu, \kappa \geq 0$, with $i>0$ only if $\mu \leq 1$ and $l>0$ only if $\nu \leq 1$.

Type $\bar{U}$. An irreducible quadrilateral $\bar{U}_{\mu \nu}^{\kappa}$ with digons $D_{i}, D_{k}, D_{l}, D_{m}$ attached to its sides $L_{1}, L_{2}, L_{3}, L_{4}$ (see Figure $14 b$ ). Here $i, k, l, m, \mu, \nu, \kappa \geq 0$, with $k>0$ only if $\nu \leq 1$ and $m>0$ only if $\mu \leq 1$.

Type $X$. An irreducible quadrilateral $X_{\mu \nu}^{\kappa}$ with digons $D_{i}, D_{k}, D_{l}, D_{m}$ attached to its sides $L_{1}, L_{2}, L_{3}, L_{4}$ (see Figure [15a). Here $i, k, l, m, \mu, \nu, \kappa \geq 0$, with $i>0$ only if $\mu \leq 1$ and $k>0$ only if $\nu \leq 1$.

Type $\bar{X}$. An irreducible quadrilateral $\bar{X}_{\mu \nu}^{\kappa}$ with digons $D_{i}, D_{k}, D_{l}, D_{m}$ attached to its sides $L_{1}, L_{2}, L_{3}, L_{4}$ (see Figure [15b). Here $i, k, l, m, \mu, \nu, \kappa \geq 0$, with $l>0$ only if $\nu \leq 1$ and $m>0$ only if $\mu \leq 1$.

Proposition 12.3. Every marked spherical quadrilateral $Q$ over partition $\mathcal{P}$ with two opposite non-integer corners, one of them marked as $a_{0}$, not containing an irreducible quadrilateral, is combinatorially equivalent to one of the following: 

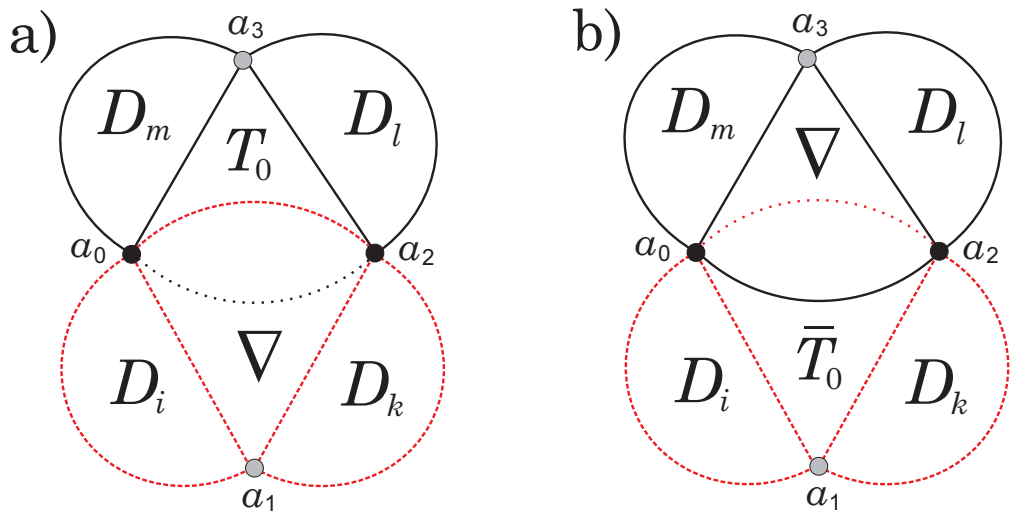

Figure 17. Equivalent quadrilaterals $T \nabla$ and $\bar{T} \nabla$.

Type $T \nabla$. An irreducible triangle $T_{0}$ with a triangle $\nabla_{j i k}$ attached to its base so that digon $D_{j}$ has a common side with $T_{0}$, and digons $D_{l}$ and $D_{m}$ attached to the sides $T_{1}$ (see Figure $16 a$ ). Here $i, j, k, l, m \geq 0$.

Type TT. Irreducible triangles $T_{\mu}$ and $T_{\nu}$, with a common integer corner at $a_{3}$, attached to the opposite sides of a digon $D_{2 \kappa}$ with digons $D_{i}$ and $D_{k}$ attached to the bases of $T_{\mu}$ and $T_{\nu}$, and digons $D_{l}$ and $D_{m}$ attached to the sides of $T_{\mu}$ and $T_{\nu}$ (see Figure $16 b$ ). Here $i, j, k, l, m \geq 0$, with $i>0$ only if $\mu=0$ and $k>0$ only if $\nu=0$.

Type $\overline{T T}$. Irreducible triangles $\bar{T}_{\mu}$ and $\bar{T}_{\nu}$, with a common integer corner at $a_{1}$, attached to the opposite sides of a digon $D_{2 \kappa}$ with digons $D_{m}$ and $D_{l}$ attached to the bases of $\bar{T}_{\mu}$ and $\bar{T}_{\nu}$, and digons $D_{i}$ and $D_{k}$ attached to the sides of $\bar{T}_{\mu}$ and $\bar{T}_{\nu}$ (see Figure 16c). Here $i, j, k, l, m \geq 0$, with $i>0$ only if $\mu=0$ and $k>0$ only if $\nu=0$.

For the type TT (resp., $\overline{T T}$ ), the integer corner $a_{1}$ (resp., $a_{3}$ ) of $\mathcal{Q}$ is mapped to a vertex $S$ (resp., $N$ ) of $\mathcal{P}$ if $\mu$ is even (resp., odd), while its other integer corner cannot be mapped to a vertex of $\mathcal{P}$. In all other cases, both integer corners of $Q$ are mapped to non-vertex points of $\mathcal{P}$.

Remark 12.4. Note that combining $\nabla$ with $\bar{T}_{0}$ (see Figure $17 \mathrm{~b}$ for the $j=0$ case) instead of $T_{0}$ as in type $T \nabla$ (see Figure 17 a for the $j=0$ case) results in a quadrilateral combinatorially equivalent to the quadrilateral of type $T \nabla$. The dotted line in Figure 17 is an interior arc of $\nabla$ which is not shown in Figure 16.

\section{Deformation of Spherical POLygons}

Deformation in a neighborhood of a corner with integer angle. In accordance with the previous sections, we consider the closed upper half-plane $\bar{H}=\{z$ : $\Im z \geq 0\} \cup\{\infty\}$ instead of the unit disk $\mathbf{D}$. Let $x \in \mathbf{R}=\partial H$ be a corner with an integer angle (i.e., an angle $\pi \alpha$ where $\alpha$ is integer), and $X=f(x)$ its image under the developing map. Then the two sides adjacent at $x$ are mapped by $f$ into a great circle, and by post-composition with a rotation of the sphere we may assume that this great circle is the real line. Then $f$ is real on an interval of the real line containing $x$. 
Suppose that $X$ is not a vertex of $\mathcal{P}$. We will show that one can deform the polygon $Q$ so that the net does not change, and the point $X$ is shifted to any position on some interval around $X$.

To do this, we take a disk $V$ centered at $X$ that contains no vertices of $\mathcal{P}$. Let $U$ be the component of $f^{-1}(V)$ that contains $x$. Let $\psi_{t}$ be orientation-preserving diffeomorphisms of $\mathbf{S}$ which are equal to identity outside $V$, map $V \cap \mathbf{R}$ onto itself, shift $X$ to $X+t$, where $t \in \mathbf{R}$ is small, and continuously depend on $t$.

Then we define

$$
g_{t}(z)= \begin{cases}\psi_{t} \circ f(z), & z \in U \\ f(z), & z \in H \backslash U .\end{cases}
$$

This is a continuous family of smooth quasiregular maps $H \rightarrow \mathbf{S}$, and by the known results on solutions of Beltrami's equation [1], one can find a continuous family of quasiconformal homeomorphisms $\psi_{t}: H \rightarrow H$ such that $\phi_{t} \circ g_{t}$ are analytic. These are the developing maps of a family of polygons which have the same partition $\mathcal{P}$, and the same net $\mathcal{Q}$ as $Q$, but the image $X$ of the vertex $x$ of $\mathcal{Q}$ is shifted on its own circle.

Notice that this procedure works also when $X$ is a vertex of $\mathcal{P}$, however the net $\mathcal{Q}$ does change under the deformation (in a controllable way; see Section 14) in the neighborhood of $x$.

In the case of a quadrilateral with two integer corners $x$ and $y$, their images $X$ and $Y$ may be on the same circle of partition $\mathcal{P}$, as in Figure 5, when the two integer corners are adjacent, or on two different circles, as in Figure 7 , when the two corners are opposite. We may assume that $S=0$ and $N=\infty$. By a fractional linear transformation, one can fix one of the two points $X$ or $Y$; then the second one gives a local parameter on the set of equivalence classes of quadrilaterals. This consideration shows that the curve in (4.10) is non-singular for $a \notin\{0,1\}$. Thus it is non-singular at all real points.

Degeneracy of spherical quadrilaterals Let us represent a marked spherical quadrilateral as a rectangle in $\mathbf{C}$ with vertices $a_{0}, a_{1}, a_{2}, a_{3}$, equipped with conformal Riemannian metric with length element $d s=\rho(z)|d z|$ of curvature +1 . We will call the sides $\left[a_{0}, a_{1}\right]$ and $\left[a_{2}, a_{3}\right]$ horizontal and the other two sides vertical. Such a quadrilateral has one conformal invariant for which we choose the extremal distance $L$ between sides $\left[a_{1}, a_{2}\right]$ and $\left[a_{3}, a_{0}\right]$. We recall the notion of extremal length and extremal distance [2].

Let $\Gamma$ be a family of curves in some region $D \subset \mathbf{C}$. Let $\lambda \geq 0$ be a measurable function in $D$. We define the $\lambda$-length of a curve $\gamma$ by

$$
\ell_{\lambda}(\gamma)=\int_{\gamma} \lambda(z)|d z|
$$

if the integral exists, and $\ell_{\lambda}(\gamma)=+\infty$ otherwise. Then we set

$$
L_{\lambda}(\Gamma)=\inf _{\gamma \in \Gamma} \ell_{\lambda}(\gamma)
$$

and

$$
A_{\lambda}(D)=\int_{D} \lambda^{2}(z) d m
$$


where $d m$ is the Euclidean area element. Then the extremal length of $\Gamma$ is defined as

$$
L(\Gamma)=\inf _{\lambda} \frac{L_{\lambda}^{2}(\Gamma)}{A_{\lambda}(D)} .
$$

The extremal length is a conformal invariant. Extremal distance between two closed sets is defined as the extremal length of the family of all curves in $D$ that connect these two sets. For a rectangle as before, the extremal distance between the vertical sides $\left[a_{1}, a_{2}\right]$ and $\left[a_{3}, a_{0}\right]$ is equal to $\left|\left[a_{0}, a_{1}\right]\right| /\left|\left[a_{1}, a_{2}\right]\right| ;$ see $[2$.

In addition to the extremal distance, we consider the ordinary intrinsic distances between the pairs of opposite sides. They are defined as the infima of $\rho$-lengths of curves contained in our quadrilateral and connecting the two sides of a pair.

Now we have the following

Lemma 13.1. Consider a sequence of marked spherical quadrilaterals whose developing maps $f$ are at most p-valent with a fixed integer $p$. If the intrinsic distance between the vertical sides is bounded from below, while the intrinsic distance between the horizontal sides tends to 0 , then the extremal distance between the vertical sides tends to $+\infty$.

Proof. Let $\gamma_{1}$ be a nearly extremal curve for the intrinsic distance between the vertical sides of $Q$, that is, the intrinsic length of $\gamma_{1}$ is at most $2 \epsilon$. Fix a point $P \in \gamma_{1}$. Choose an arbitrary (large) number $M>0$, and denote the intrinsic distance by $d$. Let $D$ be the "annulus" with respect to the intrinsic metric of radii $r_{1}=2 \epsilon$ and $r_{2}=M \epsilon$ centered at $P$, that is,

$$
D=\{z \in \bar{Q}: 2 \epsilon \leq d(z, P)<M \epsilon\} .
$$

Let $\epsilon$ be so small that $M \epsilon<\pi / 2$. and

$$
2(M \epsilon+\epsilon)<c,
$$

where $c$ is a positive lower bound of the intrinsic distance between the vertical sides. As $f$ is $p$-valent, the intrinsic area of all intrinsic disks $B(r)$ satisfies

$$
\text { area } B(r) \leq 2 \pi p r^{2} \text {. }
$$

Let $\Gamma$ be the family of curves in $Q$ connecting the horizontal sides. Every curve $\gamma \in \Gamma$ must intersect $\gamma_{1}$ and both "circles" of the annulus $D$ :

$$
C_{1}=\{z \in \bar{Q}: d(z, P)=2 \epsilon\} \quad \text { and } \quad C_{2}=\{z \in \bar{Q}: d(z, P)=M \epsilon\} .
$$

Thus $\gamma$ contains a curve of the family $\Gamma^{\prime}$ in $D$ which connect the inner "circle" $C_{1}$ to the outer "circle" $C_{2}$. It follows that $L(\Gamma) \geq L\left(\Gamma^{\prime}\right)$. The extremal length $L\left(\Gamma^{\prime}\right)$ for metric annuli with metrics satisfying (13.2) is estimated in [4, Lemma 6]:

$$
L\left(\Gamma^{\prime}\right) \geq \frac{\log \left(r_{2} / r_{1}\right)}{32 \pi} .
$$

Substituting our values $r_{1}=2 \epsilon$ and $r_{2}=M \epsilon$ we obtain

$$
1 / L(\Gamma)<1 / L\left(\Gamma^{\prime}\right)<(32 \pi) / \log (M / 2),
$$

which proves the statement as $M$ is arbitrarily large.

Example 13.2. Let $Q$ be one of the quadrilaterals with two adjacent non-integer corners $a_{0}$ and $a_{1}$ (see Figure 11).

The images $X$ and $Y$ of the integer corners $a_{2}$ and $a_{3}$ of the quadrilateral $Q$ in Figure 11k under its developing map cannot be vertices of $\mathcal{P}$. They belong to 
the same arc of a circle of $\mathcal{P}$ as shown in Figure 5. The two integer corners are connected by an arc of order 1 of the net of $Q$, thus when their images $X$ and $Y$ converge to a common point, remaining at a finite distance from the vertices of $\mathcal{P}$, the intrinsic distance between the "vertical" sides $L_{2}$ and $L_{4}$ of $Q$ tends to 0 , while the distance between its "horizontal" sides $L_{1}$ and $L_{3}$ does not tend to 0 . Lemma 13.1 implies that the extremal distance between $L_{2}$ and $L_{4}$ tends to 0 . The corner $a_{3}$ of $Q$ is connected by an arc of order 1 to its corner $a_{0}$ but not to its corner $a_{1}$. Similarly, $a_{2}$ is connected to $a_{1}$ but not to $a_{0}$. Lemma 13.1 implies that, when either $Y$ converges to the image of $a_{0}$ or $X$ converges to the image of $a_{1}$, the extremal distance between $L_{2}$ and $L_{4}$ tends to $\infty$.

For a quadrilateral $Q$ in Figure 11a (resp., Figure 11b) the image $Y$ of its corner $a_{3}$ (resp., the image $X$ of its corner $a_{2}$ ) cannot be a vertex of $\mathcal{P}$. When $Y$ (resp., $X$ ) remains at a finite distance from vertices of $\mathcal{P}$, and $X$ converges to $Y$ (resp., $Y$ converges to $X$ ), the same argument as above implies that the extremal distance between the sides $L_{2}$ and $L_{4}$ of $Q$ tends to 0 . If $Y$ (resp., $X$ ) tends to the image of $a_{0}$ (resp., $a_{1}$ ) and $X$ (resp., $Y$ ) remains at a finite distance from the vertices of $P$, Lemma 13.1 implies that the extremal distance between the sides $L_{2}$ and $L_{4}$ of $Q$ tends to $\infty$.

Example 13.3. Let $Q$ be a quadrilateral of type $U$ (see Figure 14 ) with $\mu=\nu=0$, with opposite non-integer corners $a_{0}$ and $a_{2}$. The images $X$ and $Y$ of its integer corners $a_{1}$ and $a_{3}$ cannot be vertices of $\mathcal{P}$. The corner $a_{1}$ (resp., $a_{3}$ ) of $Q$ is connected by an arc of order 1 of its net to each of its non-integer corners. When $Y$ (resp., $X$ ) remains at a finite distance from the vertices of $\mathcal{P}$ and $X$ (resp., $Y$ ) converges to the image of $a_{0}$ (resp., $a_{2}$ ), the distance between the sides $L_{2}$ and $L_{4}$ of $Q$ tends to 0 while the distance between its sides $L_{1}$ and $L_{3}$ does not tend to 0 . Lemma 13.1 implies that the extremal distance between $L_{2}$ and $L_{4}$ tends to 0 . When $Y$ (resp., $X$ ) remains at a finite distance from the vertices of $\mathcal{P}$ and $X$ (resp., $Y$ ) converges to the image of $a_{2}$ (resp., $a_{0}$ ), the distance between $L_{1}$ and $L_{3}$ of $Q$ tends to 0 while the distance between $L_{2}$ and $L_{4}$ does not tend to 0 . Lemma 13.1 implies that the extremal distance between $L_{2}$ and $L_{4}$ tends to $\infty$.

Note that, for given $i, k, l, m$ and $\kappa$, this quadrilateral is combinatorially equivalent to a quadrilateral of one of the types $\bar{U}, X, \bar{X}$ with $\mu=\nu=0$ and the same $i, k, l, m$ and $\kappa$.

Similar arguments show that, for a quadrilateral $Q$ of type $T \nabla$ (see Figure 16a), the extremal distance between $L_{2}$ and $L_{4}$ tends to 0 when either the image $X$ of $a_{1}$ tends to the image $N$ of $a_{0}$ or the image $Y$ of $a_{3}$ tends to the image $S$ of $a_{2}$, and tends to $\infty$ when either $X$ tends to $S$ or $Y$ tends to $N$.

Example 13.4. Let $Q$ be a quadrilateral of type $U$ (see Figure 14a) with $\mu>0$, $\nu>0, i=l=0$, with opposite non-integer corners $a_{0}$ and $a_{2}$. Then integer corner $a_{1}$ (resp., $a_{3}$ ) of $Q$ is connected by an arc of order 1 of its net to $a_{2}$ but not to $a_{0}$ (resp., to $a_{0}$ but not to $a_{2}$ ). The images $X$ and $Y$ of $a_{1}$ and $a_{3}$ cannot be vertices of $\mathcal{P}$. When $Y$ converges to the image $N$ of $a_{0}$ and $X$ remains at a positive distance from the vertices of $\mathcal{P}$, the distance between sides $L_{1}$ and $L_{3}$ of $Q$ tends to 0 , while the distance between $L_{2}$ and $L_{4}$ does not tend to 0 . Lemma 13.1 implies that the extremal distance between $L_{2}$ and $L_{4}$ tends to $\infty$. When $Y$ converges to the vertex $S$ of $\mathcal{P}$ and $X$ remains at a positive distance from the vertices of $\mathcal{P}$, the distance between sides $L_{1}$ and $L_{3}$ of $Q$ tends to 0 , while the distance between $L_{2}$ and $L_{4}$ does not tend to 0 , because $a_{3}$ is connected by an arc of order 1 to a vertex $q \in L_{1}$ 
of the net of $Q$ that is mapped to $S$, but is not connected by an arc of order 1 to any point on $L_{2}$ mapped to $S$. Similarly, when $X$ converges to any of the two vertices of $\mathcal{P}$, the distance between sides $L_{1}$ and $L_{3}$ of $Q$ tends to 0 , while the distance between $L_{2}$ and $L_{4}$ does not tend to 0 . Lemma 13.1 implies that the extremal distance between $L_{2}$ and $L_{4}$ tends to $\infty$ for each possible degeneration of $Q$.

\section{Chains of QUADrilaterals}

Let $Q$ be a marked quadrilateral with non-integer corners $a_{0}$ and $a_{2}$, and with integer corners $a_{1}$ and $a_{3}$ mapped to the points $X$ and $Y$, respectively, which are not vertices of $\mathcal{P}$. Let $\mathcal{Q}$ be the net of $Q$. When one of those points (say, $X$ ) approaches a vertex of $\mathcal{P}$ (say, $N$ ), and the combinatorial class of $Q$ is fixed, the following options are available.

(a) $Q$ degenerates (see section [13) so that the distance between its opposite sides $L_{1}$ and $L_{3}$ tends to 0 , while the distance between its sides $L_{2}$ and $L_{4}$ does not tend to 0 . Lemma 13.1 implies that the extremal distance between $L_{2}$ and $L_{4}$ tends to $\infty$. This happens when $\mathcal{Q}$ has an arc of order 1 connecting $a_{1}$ with a point on $L_{3}$ mapped to $N$, but does not have an arc of order 1 connecting $a_{1}$ to a point on $L_{4}$ mapped to $N$.

(b) $Q$ degenerates so that the distance between its opposite sides $L_{2}$ and $L_{4}$ tends to 0 , while the distance between its sides $L_{1}$ and $L_{3}$ does not tend to 0. Lemma 13.1 implies that the extremal distance between $L_{2}$ and $L_{4}$ tends to 0 . This happens when $\mathcal{Q}$ has an arc of order 1 connecting $a_{1}$ with a point on $L_{4}$ mapped to $N$, but does not have an arc of order 1 connecting $a_{1}$ to a point on $L_{3}$ mapped to $N$.

(c) $Q$ does not degenerate, but converges to a quadrilateral $Q^{\prime}$ with the corner $a_{1}$ mapped to the vertex $N$ of $\mathcal{P}$. This happens when $\mathcal{Q}$ does not have an arc of order 1 connecting $a_{1}$ with a point on one of the sides $L_{3}$ and $L_{4}$ mapped to $N$.

(d) $Q$ degenerates so that both distances, between $L_{1}$ and $L_{3}$ and between $L_{2}$ and $L_{4}$, tend to 0 . This happens when $\mathcal{Q}$ has arcs of order 1 connecting $a_{1}$ with points on $L_{3}$ and on $L_{4}$ mapped to $N$ (or an arc of order 1 connecting $a_{1}$ with $a_{3}$, when $a_{3}$ is mapped to $\left.N\right)$.

In the case (c), and in the corresponding cases with $Y$ instead of $X$ and/or $S$ instead of $N$, we say that $Q$ and $Q^{\prime}$ are adjacent. Note that a quadrilateral $Q^{\prime}$ of type either $T T$ or $\overline{T T}$ (see Figure 16 bc) has exactly one integer corner mapped to a vertex of $\mathcal{P}$, and there are two distinct combinatorial classes of quadrilaterals adjacent to $Q^{\prime}$.

Definition 14.1. For $k>0$, a sequence $Q_{0}, Q_{1}^{\prime}, Q_{1}, \ldots, Q_{k}^{\prime}, Q_{k}$ of quadrilaterals with distinct combinatorial types, where any two consecutive quadrilaterals are adjacent, and each of the terminal quadrilaterals $Q_{0}$ and $Q_{k}$ has only one adjacent quadrilateral, is called a chain of the length $k$. A quadrilateral $Q_{0}$ having no adjacent quadrilaterals is called a chain of length 0 .

If both cases (a) and (b) are possible for degeneration of $Q_{0}$ and $Q_{k}$, then the chain is called an ab-chain. If only the case (a) is possible, the chain is an aa-chain. If only the case (b) is possible, the chain is a bb-chain.

Example 14.2. It follows from Example 13.3 that a quadrilateral of type $U$ with $\mu=\nu=0$ is an ab-chain of length 0 . For given $i, k, l, m$ and $\kappa$, this quadrilateral 
is combinatorially equivalent to a quadrilateral of one of the types $\bar{U}, X, \bar{X}$ with $\mu=\nu=0$ and the same $i, k, l, m$ and $\kappa$.

A quadrilateral of type $U$ with $\mu=\nu=1, i>0$ and $l>0$ is an ab-chain of length 0 . A quadrilateral of type $\bar{U}$ with $\mu=\nu=1, k>0$ and $m>0$ is an ab-chain of length 0 .

A quadrilateral of type $X$ with $\mu=\nu=1, i>0$ and $k>0$ is an ab-chain of length 0 . A quadrilateral of type $\bar{X}$ with $\mu=\nu=1, l>0$ and $m>0$ is an ab-chain of length 0 .

It follows from Example 13.4 that a quadrilateral of type $U$ with $\mu>0, \nu>0$, $i=l=0$ is an aa-chain of length 0 . Similarly, a quadrilateral of type $\bar{U}$ with $\mu>0$, $\nu)>0, k=m=0$ is a bb-chain of length 0 .

It follows from Example 13.3 that a quadrilateral of type $T \nabla$ is an ab-chain of length 0 .

Lemma 14.3. Any quadrilateral with opposite integer corners which is a chain of length 0 is combinatorially equivalent to one of the quadrilaterals in Example 14.2.

Proof. One can check directly that any quadrilateral with opposite non-integer corners other than one of the quadrilaterals in Example 14.2 is either one of the quadrilaterals of types $T T$ and $\bar{T} \bar{T}$ or adjacent to a quadrilateral of type either $T T$ or $\bar{T} \bar{T}$.

Example 14.4. A chain of quadrilaterals of length 2 is shown in Figure 18, The quadrilateral $Q_{1}$ in Figure [18c is the same as the quadrilateral $I$ in Figure 13 a. It can be represented either as $X_{01}$ with a digon $D_{1}$ attached to its side $L_{2}$ or as $\bar{X}_{01}$ with a digon $D_{1}$ attached to its side $L_{3}$. The quadrilateral $Q_{0}$ in Figure $18 \mathrm{a}$ is $X_{10}=U_{10}$ with a digon $D_{1}$ attached to its side $L_{2}$. The quadrilateral $Q_{2}$ in Figure [18 is $\bar{X}_{10}=\bar{U}_{10}$ with a digon $D_{1}$ attached to its side $L_{3}$. The quadrilateral $Q_{1}^{\prime}$ in Figure 18b is a union of two triangles $T_{0}$ and a digon $D_{1}$. The quadrilateral $Q_{2}^{\prime}$ in Figure $18 \mathrm{~d}$ is a union of two triangles $\bar{T}_{0}$ and a digon $D_{1}$.

If the point $X$ to which the corner $a_{1}$ of $Q_{0}$ maps approaches $N$, the distance between the sides $L_{1}$ and $L_{3}$ (but not of $L_{2}$ and $L_{4}$ ) tends to 0 (case a). The same happens if the point $Y$ to which the corner $a_{3}$ of $Q_{0}$ maps approaches $S$. If $X$ approaches $S$, the quadrilateral $Q_{0}$ converges to $Q_{1}^{\prime}$ (case c). If $Y$ approaches $N$, both distances (between $L_{1}$ and $L_{3}$, and between $L_{2}$ and $L_{4}$ ) tend to 0 (case d). If the point $X$ to which the corner $a_{1}$ of $Q_{1}$ maps approaches $N$, both distances (between $L_{1}$ and $L_{3}$, and between $L_{2}$ and $L_{4}$ ) tend to 0 (case d). The same happens if the point $Y$ to which the corner $a_{3}$ of $Q_{1}$ maps approaches $N$. If $X$ approaches $S$, the quadrilateral $Q_{1}$ converges to $Q_{1}^{\prime}$ (case c). If $Y$ approaches $S$, the quadrilateral $Q_{1}$ converges to $Q_{2}^{\prime}$ (case c).

If the point $Y$ to which the corner $a_{3}$ of $Q_{2}$ maps approaches $N$, the distance between the sides $L_{2}$ and $L_{4}$ (but not of $L_{1}$ and $L_{3}$ ) tends to 0 (case b). Thus the chain in Figure 18 is an ab-chain.

Example 14.5. A chain of quadrilaterals of length 1 is shown in Figure 19, The quadrilateral $Q_{1}^{\prime}$ in Figure 19b has type $T T$ (see Figure 16b) with $\mu=1, \nu=0$, $\kappa=1, i=l=m=0$ and $k=1$.

The quadrilateral $Q_{0}$ in Figure 19a has type $U$ (see Figure 14) with $\mu=\nu=1$, $\kappa=1, i=k=m=0$ and $l=1$. When the image $X$ of the integer corner $a_{1}$ of $Q_{0}$ approaches the image $N$ of its non-integer corner $a_{0}$, the quadrilateral $Q_{0}$ does not degenerate, and converges to the quadrilateral $Q_{1}^{\prime}$. When $X$ approaches the image 

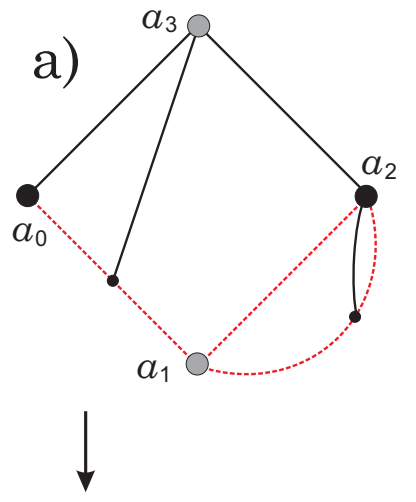

c)
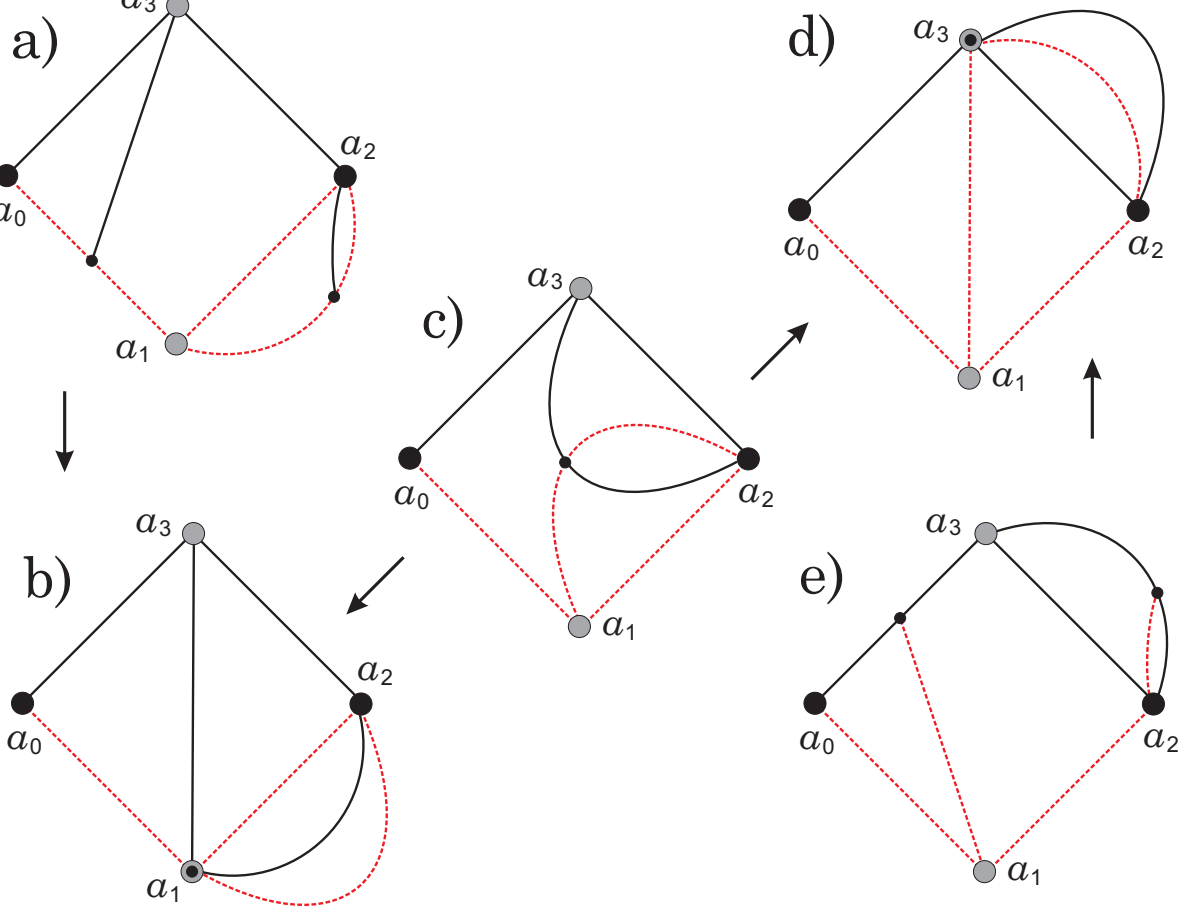

Figure 18. An ab-chain of quadrilaterals of length 2.
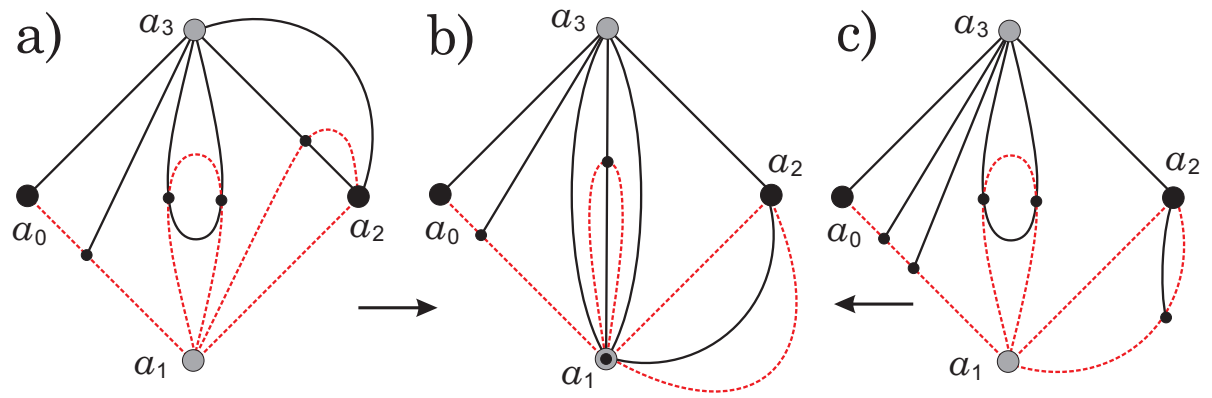

FiguRE 19. An aa-chain of quadrilaterals of length 1.

$S$ of the non-integer corner $a_{2}$ of $Q_{0}$, the quadrilateral $Q_{0}$ degenerates so that the distance between its sides $L_{1}$ and $L_{2}$ tends to 0 , while the distance between its sides $L_{2}$ and $L_{4}$ does not tend to 0 . Lemma 13.1 implies that the extremal distance between $L_{2}$ and $L_{4}$ tends to $\infty$.

The quadrilateral $Q_{1}$ in Figure [19, has type $U$ with $\mu=2, \nu=0, \kappa=1$, $i=l=m=0$, and $k=1$. It is combinatorially equivalent to a quadrilateral of type $X$ with the same values of $\mu, \nu, \kappa, i, l, m, k$. The same argument shows that $Q_{1}$ either converges to $Q_{1}^{\prime}$ or degenerates so that the extremal distance between its sides $L_{2}$ and $L_{4}$ tends to $\infty$.

Thus the chain in Figure 19 is an aa-chain. 


\section{Alternative proof of Theorem 5.3}

The properties of nets described in sections 6[14 allow us to give an alternative proof of cases (i) and (ii) of Theorem 5.3. computing the lower bound for the number of marked quadrilaterals with given angles and modulus, when only two angles are non-integer.

In the case of adjacent non-integer corners (case (i) of Theorem 5.3), Example 13.2 implies that, moving the images of integer corners of a quadrilateral with fixed angles in a given combinatorial equivalence class, one can obtain a quadrilateral with the modulus (extremal distance between two of its opposite sides) attaining any value between 0 and infinity. Thus the number of quadrilaterals with the given angles and modulus is bounded from below by the number of distinct combinatorial classes of the quadrilaterals. Proposition 11.3 implies that the number of such classes equals the number of real solutions in Theorem 5.3 (i).

In case of opposite non-integer corners, one has to count ab-chains of quadrilaterals instead of single combinatorial equivalence classes. It follows from Definition 14.1 that, for any fixed angles, any admissible ab-chain $C$, and any value of the modulus, there exists a quadrilateral combinatorially equivalent to one of the quadrilaterals in $C$ with the given angles and modulus.

Instead of counting ab-chains directly, we note that the total number of chains equals to the number of complex solutions of equation (4.10), which is also the number of metrics in Theorem 4.1 and the number of real solutions in Theorem 5.3 (i). Indeed, due to Proposition 5.2, the number of real solutions of (4.10) for a small $a>0$ equals the number of its complex solutions. Every ab-chain gives one solution, and every aa-chain gives two solutions (as it has both ends at $a=0$ ). Since the number of bb-chains equals the number of aa-chains by reflection symmetry, the total number of solutions equals the total number of chains.

This implies that the number of ab-chains equals the number of all chains minus twice the number of aa-chains, and it is enough to count aa-chains (or bb-chains). We perform that count in this section. Specifically, we count the aa-chains of marked quadrilaterals having given orders $A_{0}, \ldots, A_{3}$ of their corners $a_{0}, \ldots, a_{3}$.

Lemma 15.1. A chain of quadrilaterals is an aa-chain (resp., a bb-chain) if and only if it contains a quadrilateral of type $U$ (resp., $\bar{U}$ ) with $\mu>0, \nu>0$ and $\min (i, l)=0$. A chain may contain at most one such quadrilateral.

Proof. Let us show first that each chain containing a quadrilateral $Q_{0}$ of type $U$ with $\mu>0, \nu>0$ and $\min (i, l)=0$ is an aa-chain. Example 13.4 shows that $Q_{0}$ itself is an aa-chain of length 0 when $i=l=0$. If $i=0$ and $l>0$, then $\nu=1$ and $Q_{0}$ is adjacent to a quadrilateral $Q_{1}^{\prime}$ of type $T T$ with $\nu=0, l$ decreased by 1 , and $k$ increased by 1 . The other quadrilateral $Q_{1}$ adjacent to $Q_{1}^{\prime}$ has type $U$ with $\nu=0, \mu$ increased by $1, l$ decreased by 1 , and $k$ increased by 1 . Both $Q_{0}$ and $Q_{1}$ can be degenerated so that the extremal distance between their sides $L_{2}$ and $L_{4}$ tends to $\infty$, thus $Q_{0}, Q_{1}^{\prime}, Q_{1}$ is an aa-chain of length 1 . An example of such a chain is considered in Example 14.5] and shown in Figure 19. The case when $i>0$ and $l=0$ follows by rotational symmetry.

This argument implies also that a chain containing a quadrilateral of type $U$ with $\mu>1, \nu=0$, and $k>0$ (or with $\mu=0, \nu>1$ and $m>0$ ) is an aa-chain of length 1 and contains a quadrilateral of type $U$ with $\mu>0, \nu>0$ and $\min (i, l)=0$. 
The proof that all other chains are not aa-chains can be done case-by-case and is not given here. One of the hardest cases is considered in Example 14.4 and shown in Figure 18,

Theorem 15.2. The number of aa-chains of quadrilaterals having given orders $A_{0}, \ldots, A_{3}$ of their corners $a_{0}, \ldots, a_{3}$ is

$$
\left[\frac{1}{2} \min \left(A_{1}, A_{3}, \delta\right)\right]
$$

where $\delta=\frac{1}{2} \max \left(0, A_{1}+A_{3}-A_{0}-A_{2}\right)$.

Proof. According to Lemma 15.1 we have to count quadrilaterals of type $U$ with $\min (\mu, \nu)>0$ and $\min (i, l)=0$ with the given orders of their corners. Note that for such a quadrilateral with $l>0$ necessarily $i=0$ and $\nu=1$, thus $\eta=$ $\left(A_{3}-A_{0}\right)-\left(A_{1}-A_{2}\right)=\mu-\nu+2 l \geq 2$. Similarly, if $i>0$, then $l=0$ and $\mu=1$, thus $\eta=\mu-\nu-2 i \leq-2$. In particular, it is enough to count quadrilaterals with $\eta \geq 0$, for which $i=0$ and $l \geq 0$. The case $\eta<0$ would then follow by rotation symmetry.

We start with the quadrilaterals $U_{\mu \nu}^{\kappa}$ with $i=l=0, \mu \geq \nu>0$ and digons $D_{k}$ and $D_{m}$ attached. Then,

$$
A_{0}=m, A_{1}=k+\nu+2 \kappa+1, A_{2}=k, A_{3}=m+\mu+2 \kappa+1 .
$$

Thus $A_{0} \geq 0, A_{2} \geq 0, A_{1} \geq A_{2}+2, A_{3} \geq A_{0}+2, A_{3}-A_{0} \geq A_{1}-A_{2} \geq 2$, and $0 \leq \kappa \leq \frac{1}{2}\left(A_{1}-A_{2}-2\right)$. This implies that the number of these quadrilaterals is $\left[\frac{1}{2}\left(A_{1}-A_{2}\right)\right]$. Also, $A_{3}-\delta=m+(\mu-\nu) / 2 \geq 0$, thus $\min \left(A_{1}, A_{3}, \delta\right)=\min \left(A_{1}, \delta\right)$ in this case.

Note that each quadrilateral is uniquely determined by the value of $\kappa$, and the possible values of $\kappa$ constitute a segment of integers with the lower end 0 and the upper end $\max (\kappa)$ corresponding to a quadrilateral with $1 \leq \nu \leq \mu$. We'll show next that either this number equals $\left[\frac{1}{2} \min \left(A_{1}, \delta\right)\right]$ or there exists a quadrilateral $Q^{\prime}$ with the same angles as $Q, l>0$, and the multiplicity of a pseudo-diagonal $\max (\kappa)+1$. Note that $\left[\frac{\delta}{2}\right]=\left[\frac{1}{2}\left(A_{1}-A_{2}\right)\right]$ if and only if $\mu+\nu \leq 5$.

If $k=0$, then $A_{2}=0$ thus there are no quadrilaterals with $l>0$ and the number of quadrilaterals is $\left[\frac{1}{2}\left(A_{1}-A_{2}\right)\right]=\left[\frac{A_{1}}{2}\right] \leq\left[\frac{\delta}{2}\right]$.

If $k \geq 1$, a quadrilateral $Q^{\prime}$ with $l>0$ exists either when $\nu=2$ and $\mu \geq 4$, in which case $\mathcal{Q}^{\prime}$ can be taken as $U_{\mu-3,1}^{\kappa+1}$ with $D_{k-1}$ attached to the side $L_{2}, D_{1}$ attached to the side $L_{3}$ and $D_{m}$ attached to the side $L_{4}$, or when $k \geq 2, \nu=1$ and $\mu \geq 5$, in which case $Q^{\prime}$ can be taken as $U_{\mu-4,1}^{\kappa+1}$ with $D_{k-2}$ attached to $L_{2}, D_{2}$ attached to $L_{3}$, and $D_{m}$ attached to $L_{4}$. In both cases, $\left[\frac{\delta}{2}\right]>\left[\frac{1}{2}\left(A_{1}-A_{2}\right)\right]$ and $\left[\frac{A_{1}}{2}\right]>\left[\frac{1}{2}\left(A_{1}-A_{2}\right)\right]$.

In the remaining case $k=\nu=1, \mu \geq 5$, we have $\left[\frac{\delta}{2}\right]>\left[\frac{A_{1}}{2}\right]=\left[\frac{1}{2}\left(A_{1}-A_{2}\right)\right]$.

Next, we consider the quadrilaterals with $i=0$ and $l>0$. For such a quadrilateral $Q$, necessarily $\nu=1$. Let $\kappa$ be the number of pseudo-diagonals of $Q$. Then the values $m=A_{0}, k=A_{1}-2 \kappa-2, l=A_{2}-k=A_{2}-A_{1}+2 \kappa+2$, and $\mu=A_{3}-A_{0}-l-2 \kappa-1=A_{3}-A_{0}+A_{1}-A_{2}-4 \kappa-3$ are uniquely determined by $\kappa$, and the conditions $k \geq 0, l \geq 1, \mu \geq 1$ imply that $2 \kappa \leq A_{1}-2,2 \kappa \geq A_{1}-A_{2}-1$ and $2 \kappa \leq \delta-2$. Thus, for the given values of $A_{0}, \ldots, A_{3}$, the available values of $\kappa$ constitute a segment in the non-negative integers which, if non-empty, has the upper end $\left[\frac{1}{2} \min \left(A_{1}, \delta\right)\right]-1$. If the lower end $\min (\kappa)$ of that segment is 0 , then 
$2 \leq A_{1}=k+2 \leq A_{2}+1$. Thus there are no quadrilaterals with the same values of $A_{0}, \ldots, A_{3}$ and $i=l=0$.

Otherwise, for any $0 \leq \kappa^{\prime}<\min (\kappa)$ there is a unique quadrilateral with the same values of $A_{0}, \ldots, A_{3}$ and $i=l=0$.

In any case, the total number of quadrilaterals with the given values of $A_{0}, \ldots, A_{3}$ equals (15.1).

\section{EXAMPLES}

In the following examples we choose the upper half-plane conformal model with corners $0,1, a, \infty$, integer angles $\alpha_{1}$ and $\alpha_{2}$ at 0 and 1, non-integer angles $\alpha_{0}$ and $\alpha_{3}$ at $a$ and $\infty$, so the Heun equation has the form

$$
y^{\prime \prime}+\left(\frac{1-\alpha_{1}}{z}+\frac{1-\alpha_{2}}{z-1}+\frac{1-\alpha_{0}}{z-a}\right) y^{\prime}+\frac{\alpha^{\prime} \alpha^{\prime \prime} z-\lambda}{z(z-1)(z-a)} y=0 .
$$

We plot the real part of the curve

$$
F(a, \lambda)=0
$$

as in (4.10) which is defined by the condition that the monodromy of Heun's equation is unitary. In our examples (16.1) is the condition of the absence of logarithms in the expansion at 0 .

The values $0<a<1$ correspond to quadrilaterals with opposite integer corners. In section 6 we did show that this curve has no real singularities when $a \notin\{0,1\}$. That it has no singularities over $a=0$ and $a=1$ follows from the form of the Jacobi matrix: when $a=0$, the matrix becomes triangular, with distinct diagonal entries. In Figure 20, the equation (4.10) is of degree 4 in $\lambda$, and it has at least two real solutions for all $a$. For $a$ close to 0 or 1 it has four distinct real solutions. In Figures 21] and 22, there are no real solutions for some values of $a$. Figures 23, 24] and 25 show that the number of real solutions can be larger than the lower estimate given by Theorem 5.3 even when $a$ is not close to either 0 or 1 .

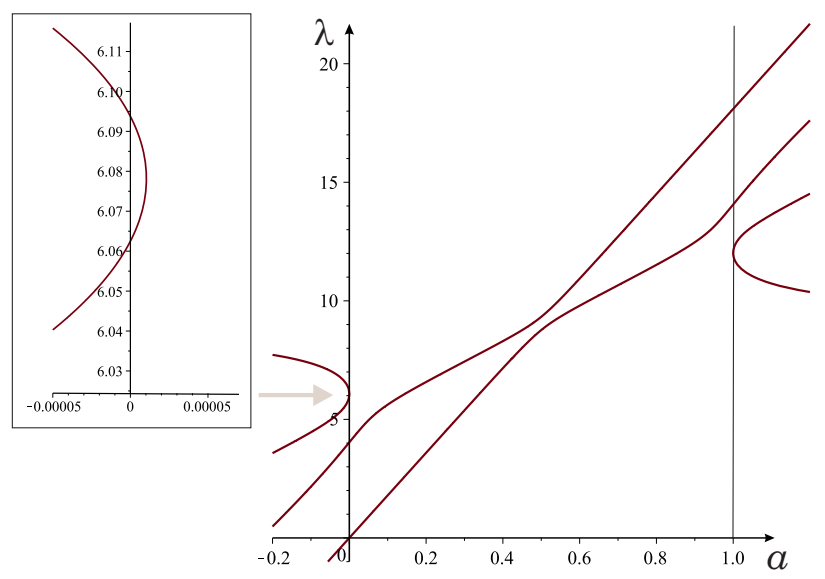

FiguRE 20. $\alpha_{1}=4, \alpha_{2}=6, \alpha_{0}=\alpha_{3}=65 / 32$. 


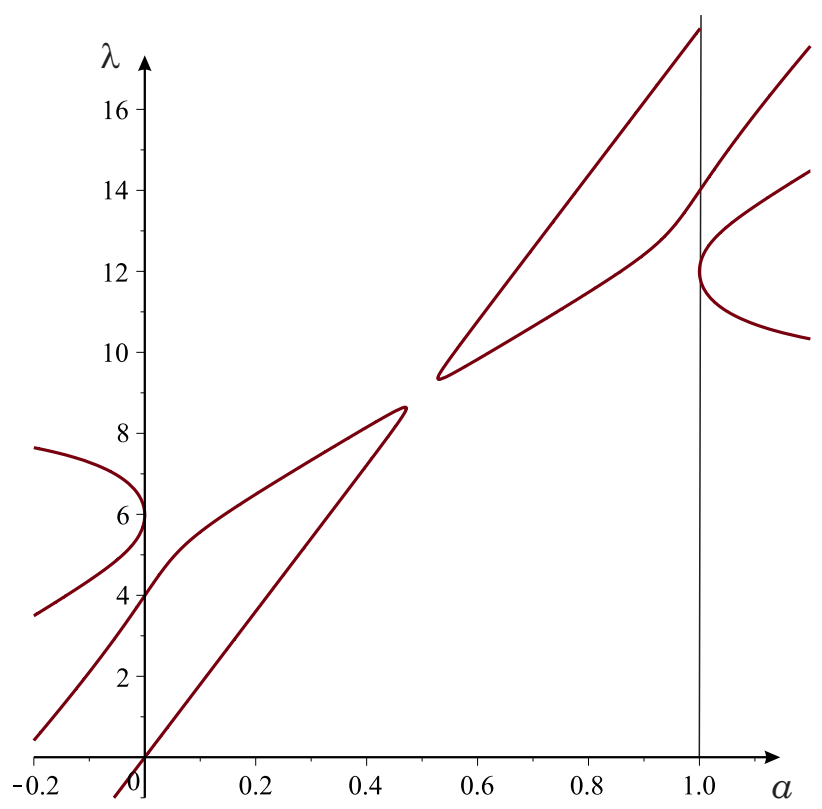

Figure 21. $\alpha_{1}=4, \alpha_{2}=6, \alpha_{0}=\alpha_{3}=255 / 128$.

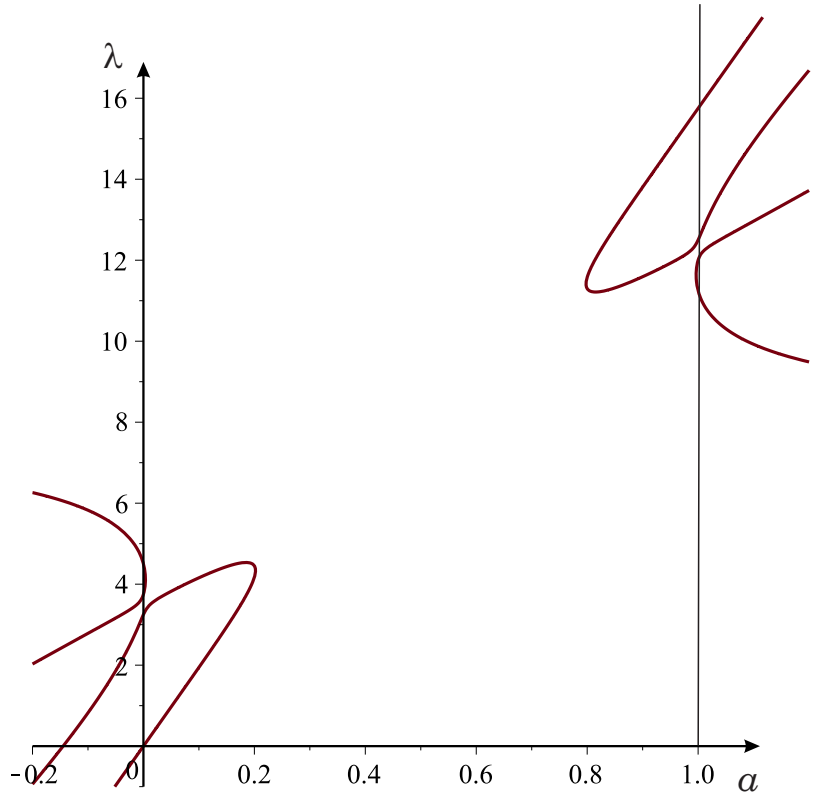

FiguRE 22. $\alpha_{1}=4, \alpha_{2}=6, \alpha_{0}=\alpha_{3}=5 / 4$. 


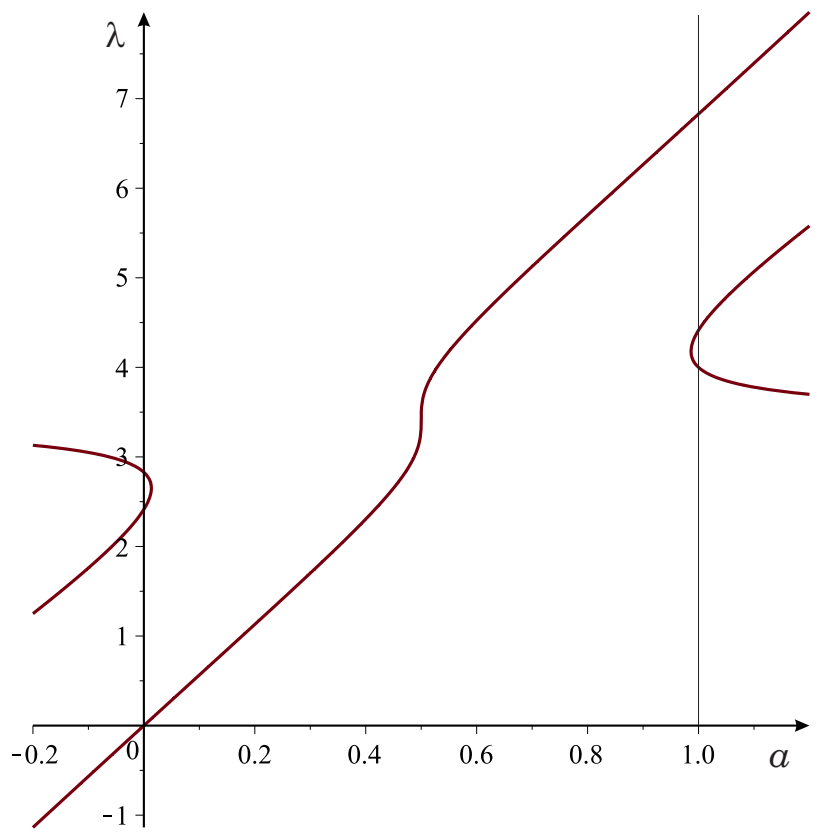

FiguRE 23. $\alpha_{1}=\alpha_{2}=3, \alpha_{0}=\alpha_{3}=\sqrt{2}$.

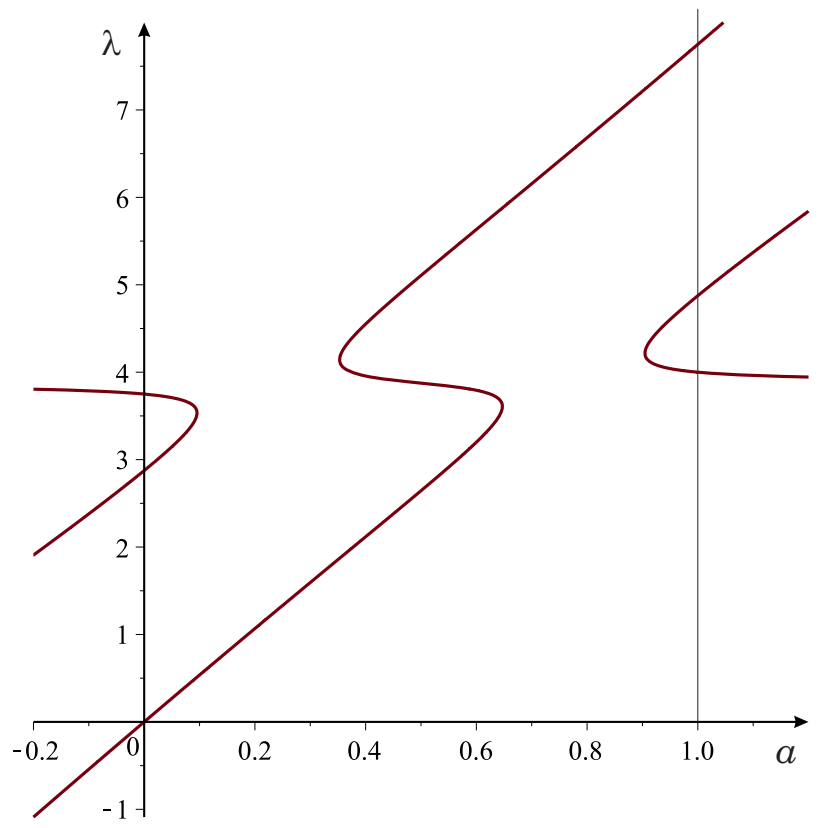

FiguRE 24. $\alpha_{1}=\alpha_{2}=3, \alpha_{0}=\alpha_{3}=15 / 8$. 


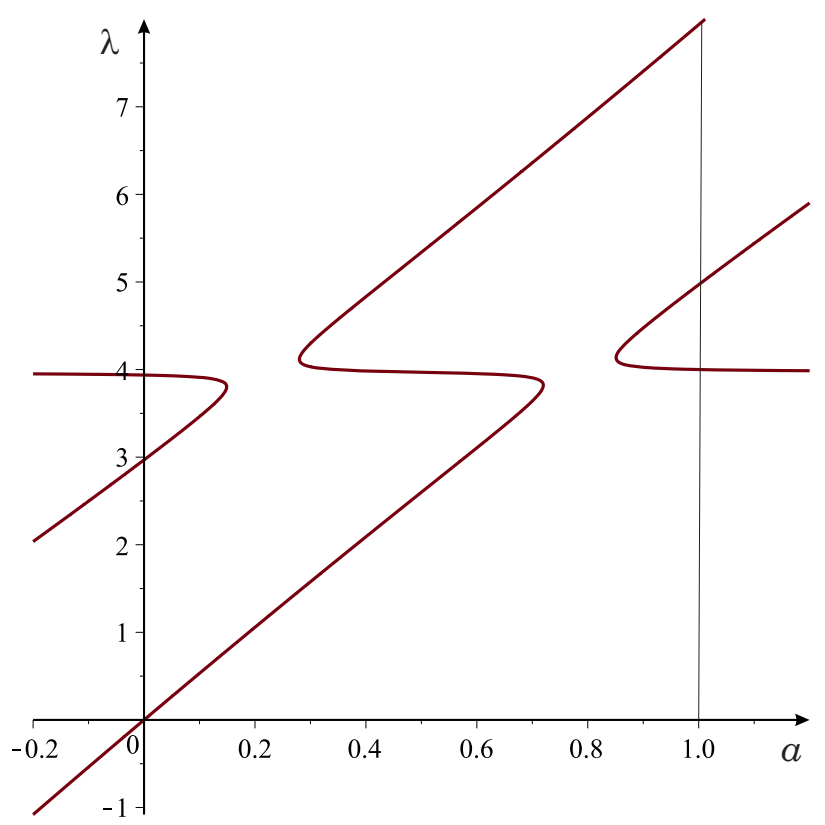

FiguRE 25. $\alpha_{1}=\alpha_{2}=3, \alpha_{0}=\alpha_{3}=63 / 32$.

\section{REFERENCES}

[1] L. V. Ahlfors, Lectures on quasiconformal mappings, 2nd ed., University Lecture Series, vol. 38, American Mathematical Society, Providence, RI, 2006. With supplemental chapters by C. J. Earle, I. Kra, M. Shishikura and J. H. Hubbard. MR2241787

[2] L. V. Ahlfors, Conformal invariants, AMS Chelsea Publishing, Providence, RI, 2010. Topics in geometric function theory; Reprint of the 1973 original; With a foreword by Peter Duren, F. W. Gehring and Brad Osgood. MR2730573

[3] I. Biswas, A criterion for the existence of a parabolic stable bundle of rank two over the projective line, Internat. J. Math. 9 (1998), no. 5, 523-533, DOI 10.1142/S0129167X98000233. MR.1644048

[4] M. Bonk and A. Eremenko, Uniformly hyperbolic surfaces, Indiana Univ. Math. J. 49 (2000), no. 1, 61-80. MR 1777037

[5] R. Buckman and N. Schmitt, Spherical polygons and unitarization, www.gang.umass.edu/ reu/2002/gon.pdf.

[6] C.-L. Chai, C.-S. Lin, and C.-L. Wang, Mean field equations, hyperelliptic curves and modular forms: I, Camb. J. Math. 3 (2015), no. 1-2, 127-274. MR3356357

[7] C.-C. Chen and C.-S. Lin, Mean field equation of Liouville type with singular data: topological degree, Comm. Pure Appl. Math. 68 (2015), no. 6, 887-947, DOI 10.1002/cpa.21532. MR.3340376

[8] Q. Chen, W. Wang, Y. Wu, and B. Xu, Conformal metrics with constant curvature one and finitely many conical singularities on compact Riemann surfaces, Pacific J. Math. 273 (2015), no. 1, 75-100, DOI 10.2140/pjm.2015.273.75. MR3290445

[9] J. Dorfmeister and M. Schuster, Construction of planar CMC 4-noids of genus $g=0$, JP J. Geom. Topol. 6 (2006), no. 3, 319-381. MR2284531

[10] J. Dorfmeister and J.-H. Eschenburg, Real Fuchsian equations and constant mean curvature surfaces, Mat. Contemp. 35 (2008), 1-25. MR.2584173

[11] A. Eremenko, Metrics of positive curvature with conic singularities on the sphere, Proc. Amer. Math. Soc. 132 (2004), no. 11, 3349-3355 (electronic), DOI 10.1090/S0002-9939-0407439-8. MR2073312 
[12] A. Eremenko and A. Gabrielov, Rational functions with real critical points and the B. and M. Shapiro conjecture in real enumerative geometry, Ann. of Math. (2) 155 (2002), no. 1, 105-129, DOI 10.2307/3062151. MR1888795

[13] A. Eremenko and A. Gabrielov, The Wronski map and Grassmannians of real codimension 2 subspaces, Comput. Methods Funct. Theory 1 (2001), no. 1, 1-25, DOI 10.1007/BF03320973. MR.1931599

[14] A. Eremenko and A. Gabrielov, An elementary proof of the B. and M. Shapiro conjecture for rational functions, Notions of positivity and the geometry of polynomials, Trends Math., Birkhäuser/Springer Basel AG, Basel, 2011, pp. 167-178, DOI 10.1007/978-3-0348-0142-3_10. MR.3051166

[15] A. Eremenko and A. Gabrielov, Counterexamples to pole placement by static output feedback, Linear Algebra Appl. 351/352 (2002), 211-218, DOI 10.1016/S0024-3795(01)00443-8. Fourth special issue on linear systems and control. MR 1917479

[16] A. Eremenko, A. Gabrielov, and V. Tarasov, Metrics with conic singularities and spherical polygons, Illinois J. Math. 58 (2014), no. 3, 739-755. MR3395961

[17] A. Eremenko, A. Gabrielov, and V. Tarasov, Spherical quadrilaterals with three non-integer angles, arXiv:1504.02928. To appear in Journal of Mathematical Physics, Analysis and Geometry.

[18] A. Eremenko, A. Gabrielov, M. Shapiro, and A. Vainshtein, Rational functions and real Schubert calculus, Proc. Amer. Math. Soc. 134 (2006), no. 4, 949-957 (electronic), DOI 10.1090/S0002-9939-05-08048-2. MR2196025

[19] S. Fujimori, Y. Kawakami, M. Kokubu, W. Rossman, M. Umehara, and K. Yamada, CMC-1 trinoids in hyperbolic 3-space and metrics of constant curvature one with conical singularities on the 2-sphere, Proc. Japan Acad. Ser. A Math. Sci. 87 (2011), no. 8, 144-149. MR2843096

[20] F. P. Gantmacher and M. G. Krein, Oscillation matrices and kernels and small vibrations of mechanical systems, Revised edition, AMS Chelsea Publishing, Providence, RI, 2002. Translation based on the 1941 Russian original; Edited and with a preface by Alex Eremenko. MR.1908601

[21] L. R. Goldberg, Catalan numbers and branched coverings by the Riemann sphere, Adv. Math. 85 (1991), no. 2, 129-144, DOI 10.1016/0001-8708(91)90052-9. MR1093002

[22] M. Heins, On a class of conformal metrics, Nagoya Math. J. 21 (1962), 1-60. MR0143901

[23] E. Hilb, Über Kleinsche Theoreme in der Theorie der linearen Differentialgleichingen, Ann. Math., 66 (1909) 215-257.

[24] E. Hilb, Über Kleinsche Theoreme in der Theorie der linearen Differentialgleichungen (2 Mitteilung), Ann. Math., 68 (1910) 24-71.

[25] A. Hurwitz, Über die Nullstellen der hypergeometrischen Funktion (German), Math. Ann. 64 (1907), no. 4, 517-560, DOI 10.1007/BF01450062. MR.1511455

[26] W. Ihlenburg, Über die geometrischen Eigenschaften der Kreisbogenvierecke, Nova Acta Leopoldina, 91 (1909) 1-79 and 5 pages of tables.

[27] W. Ihlenburg, Ueber die gestaltlichen Vergältnisse der Kreisbogenvierecke, Göttingen Nachrichten, (1908) 225-230.

[28] F. Klein, Ueber die Nullstellen der hypergeometrischen Reihe (German), Math. Ann. 37 (1890), no. 4, 573-590, DOI 10.1007/BF01724773. MR.1510659

[29] F. Klein, Bemerkungen zur Theorie der linearen Differentialgleichungen zweiter Ordnung (German), Math. Ann. 64 (1907), no. 2, 175-196, DOI 10.1007/BF01449891. MR1511433

[30] F. Luo and G. Tian, Liouville equation and spherical convex polytopes, Proc. Amer. Math. Soc. 116 (1992), no. 4, 1119-1129, DOI 10.2307/2159498. MR1137227

[31] G. Mondello and D. Panov, Spherical metrics with conical singularities on a 2-sphere: angle constraints, arXiv:1505.01994.

[32] E. Mukhin, V. Tarasov, and A. Varchenko, The B. and M. Shapiro conjecture in real algebraic geometry and the Bethe ansatz, Ann. of Math. (2) 170 (2009), no. 2, 863-881, DOI 10.4007/annals.2009.170.863. MR2552110

[33] E. Mukhin, V. Tarasov, and A. Varchenko, On reality property of Wronski maps, Confluentes Math. 1 (2009), no. 2, 225-247, DOI 10.1142/S1793744209000092. MR2561998

[34] E. Picard, De l'equation $\Delta u=k e^{u}$ sur une surface de Riemann fermée, J. Math. Pures Appl 9 (1893) 273-292.

[35] E. Picard, De l'equation $\Delta u=e^{u}$, J. Math Pures Appl., 4 (1898) 313-316. 
[36] E. Picard, De l'intégration de l'équation $\Delta u=e^{u}$ sur une surface de Riemann fermée (French), J. Reine Angew. Math. 130 (1905), 243-258, DOI 10.1515/crll.1905.130.243. MR.1580684

[37] E. Picard, Quelques applications analytiques de la théorie des courbes et des surfaces algébriques, Gauthier-Villars, Paris, 1931.

[38] L. Pontrjagin, Hermitian operators in spaces with indefinite metric (Russian, with English summary), Bull. Acad. Sci. URSS. Sér. Math. [Izvestia Akad. Nauk SSSR] 8 (1944), 243-280. MR.0012200

[39] C. D. Hodgson and I. Rivin, A characterization of compact convex polyhedra in hyperbolic 3-space, Invent. Math. 111 (1993), no. 1, 77-111, DOI 10.1007/BF01231281. MR.1193599

[40] A. Ronveaux (ed.), Heun's differential equations, Oxford Science Publications, The Clarendon Press, Oxford University Press, New York, 1995. With contributions by F. M. Arscott, S. Yu. Slavyanov, D. Schmidt, G. Wolf, P. Maroni and A. Duval. MR.1392976

[41] H. P. de Saint-Gervais, Uniformisation des surfaces de Riemann (French), ENS Éditions, Lyon, 2010. Retour sur un théorème centenaire. [A look back at a 100-year-old theorem]; The name of Henri Paul de Saint-Gervais covers a group composed of fifteen mathematicians: Aurélien Alvarez, Christophe Bavard, François Béguin, Nicolas Bergeron, Maxime Bourrigan, Bertrand Deroin, Sorin Dumitrescu, Charles Frances, Étienne Ghys, Antonin Guilloux, Frank Loray, Patrick Popescu-Pampu, Pierre Py, Bruno Sévennec, and Jean-Claude Sikorav. MR 2768303

[42] I. Scherbak, Rational functions with prescribed critical points, Geom. Funct. Anal. 12 (2002), no. 6, 1365-1380, DOI 10.1007/s00039-002-1365-4. MR1952932

[43] A. Schönflies, Ueber Kreisbogendreiecke und Kreisbogenvierecke (German), Math. Ann. 44 (1894), no. 1, 105-124, DOI 10.1007/BF01446976. MR1510837

[44] A. Schönflies, Ueber Kreisbogenpolygone (German), Math. Ann. 42 (1893), no. 3, 377-408, DOI 10.1007/BF01444164. MR1510783

[45] V. I. Smirnov, The problem of inversion of a linear differential equation of second order with four singularities, Petrograd, 1918 (Russian). Reproduced in V. I Smirnov, Selected works, vol. 2, Analytic theory of ordinary differential equations, St. Peterburg University, St. Peterburg, 1996.

[46] V. Smirnoff, Sur les équations différentialles linéaires du second ordre et la théorie des fonctions automorphes, Bull. Sci. Math., 45 (1921) 93-120, 126-135.

[47] G. Tarantello, Analytical, geometrical and topological aspects of a class of mean field equations on surfaces, Discrete Contin. Dyn. Syst. 28 (2010), no. 3, 931-973, DOI 10.3934/dcds.2010.28.931. MR2644774

[48] M. Troyanov, Prescribing curvature on compact surfaces with conical singularities, Trans. Amer. Math. Soc. 324 (1991), no. 2, 793-821, DOI 10.2307/2001742. MR1005085

[49] M. Troyanov, Metrics of constant curvature on a sphere with two conical singularities, Differential geometry (Peñíscola, 1988), Lecture Notes in Math., vol. 1410, Springer, Berlin, 1989, pp. 296-306, DOI 10.1007/BFb0086431. MR.1034288

[50] M. Umehara and K. Yamada, Metrics of constant curvature 1 with three conical singularities on the 2-sphere, Illinois J. Math. 44 (2000), no. 1, 72-94. MR1731382

[51] E. B. Van Vleck, A determination of the number of real and imaginary roots of the hypergeometric series, Trans. Amer. Math. Soc. 3 (1902), no. 1, 110-131, DOI 10.2307/1986319. MR.1500590

[52] M. Yoshida, A naive-topological study of the contiguity relations for hypergeometric functions, PDEs, submanifolds and affine differential geometry, Banach Center Publ., vol. 69, Polish Acad. Sci., Warsaw, 2005, pp. 257-268, DOI 10.4064/bc69-0-20. MR2189572

Department of Mathematics, Purdue University, West Lafayette, Indiana 47907-2067

Department of Mathematics, Purdue University, West Lafayette, Indiana 47907-2067

Department of Mathematics, IUPUi, Indianapolis, Indiana 46202-3216 — and — St. Petersburg branch of Steklov Mathematical Institute, 27, Fontanka, 191023 St. PeTERSBURG, Russia 